\title{
Models for Hylomorphism
}

\section{Bruno Jacinto $^{1} \cdot$ A. J. Cotnoir ${ }^{1}$}

Received: 18 May 2018 / Accepted: 16 January 2019 / Published online: 11 March 2019

(C) The Author(s) 2019

\begin{abstract}
In a series of papers (Fine et al., 1982; Fine, Noûs 28(2), 137-158; 1994, Midwest Studies in Philosophy, 23, 61-74, 1999) Fine develops his hylomorphic theory of embodiments. In this article, we supply a formal semantics for this theory that is adequate to the principles laid down for it in (Midwest Studies in Philosophy, 23, 61-74, 1999). In Section 1, we lay out the theory of embodiments as Fine presents it. In Section 2, we argue on Cantorian grounds that the theory needs to be stabilized, and sketch some ways forward, discussing various choice points in modeling the view. In Section 3, we develop a formal semantics for the theory of embodiments by constructing embodiments in stages and restricting the domain of the second-order quantifiers. In Section 4 we give a few illustrative examples to show how the models deliver Finean hylomorphic consequences. In Section 5, we prove that Fine's principles are sound with respect to this semantics. In Section 6 we present some inexpressibility results concerning Fine's various notions of parthood and show that in our formal semantics these notions are all expressible using a single mereological primitive. In Section 7, we prove several mereological results stemming from the model theory, showing that the mereology is surprisingly robust. In Section 8, we draw some philosophical lessons from the formal semantics, and in particular respond to Koslicki's (2008) main objection to Fine's theory. In the appendix we present proofs of the inexpressibility results of Section 6.
\end{abstract}

Keywords Objects · Parthood · Composition · Mereology · Hylomorphism · Rigid embodiment · Variable embodiment · Qua-objects · Atomism · Gunk · Junk · Aristotle $\cdot$ Neo-Aristotelian · Cantor · Cardinality · Iterative · Hierarchy

Bruno Jacinto

bmj@st-andrews.ac.uk

https://www.brunojacinto.net/

\section{A. J. Cotnoir}

ac117@st-andrews.ac.uk

http://www.st-andrews.ac.uk/ ac117

1 Department of Philosophy, University of St Andrews, Edgecliffe G07, The Scores, St Andrews, Fife KY16 9AL, UK 


\section{The Theory of Embodiments}

Fine [10] presents a number of arguments that standard mereology offers an incorrect answer to how the existence conditions of wholes are determined in terms of their parts. He proposes his theory of embodiments as a more satisfactory account. The theory is broadly Aristotelian as it stems from a hylomorphic conception of objects in that embodiments have both formal and material parts.

Fine's theory presupposes a distinction between timeless and temporary parthood. Timeless parts are ones for which it does not make sense to ask for how long some objects have been parts of a whole. For instance, Fine takes the parts of a ham sandwich to be timeless in this sense. On his view it makes no sense to ask for how long have the slices of bread been parts of the ham sandwich. Temporary parts are ones for which it does makes sense to ask for how long some objects have been parts of a whole. For instance, Fine takes the parts of a car to be temporary in this sense. On his view, it makes sense to ask for how long has a car's (current) carburettor been a part of the car.

We begin by giving an informal presentation of Fine's view, following the most developed presentation of it in Fine [10]. Fine's theory of embodiments is a theory of objects of two kinds: rigid embodiments and variable embodiments. First, consider Fine's conception of rigid embodiments:

Rigid Embodiments Given objects $a, b, c, \ldots$ and a relation $R$ (holding contingently of them), there is an object $e=a, b, c, \ldots / R$ which is an amalgam or composite of $a, b, c, \ldots$ and $R$.

Here $e$ is the rigid embodiment, $R$ is the principle of rigid embodiment, and / denotes some sort of operation of rigid embodiment. The key idea is that the objects $a, b, c \ldots$ and $R$ form a whole by their standing in that relation. As Fine writes,

The relation $R$ preserves its predicative role and somehow serves to modify or qualify the components. However, the result of the modification is not a fact or state. It is a whole, whose components are linked by the relation, rather than the fact or state of the components being so linked. (p. 65)

Limit cases of rigid embodiments $a / P$ with exactly one object and a monadic property $P$, are what Fine [8] calls 'qua-objects'.

Rigid embodiments satisfy the following principles.

(R1) Existence: $a, b, c, \ldots / R$ exists at $t$ iff $R$ holds of $a, b, c, \ldots$ at $t$.

(R2) Location: If $e=a, b, c, \ldots / R$ exists at $t$ then $e$ is located a point $p$ at $t$ iff at least one of $a, b, c, \ldots$ located at $p$.

(R3) Identity: For $e=a, b, c, \ldots / R$ and $e^{\prime}=a^{\prime}, b^{\prime}, c^{\prime}, \ldots / R^{\prime}, e=e^{\prime}$ iff $a=a^{\prime}$, $b=b^{\prime}, c=c^{\prime}, \ldots$ and $R=R^{\prime}$.

(R4) Parthood: $a, b, c, \ldots$ are (timeless) parts of $a, b, c, \ldots / R$.

(R5) Constituent Aspect: $R$ is a (timeless) part of $a, b, c, \ldots / R$.

(R6) That's All Folks: Any (timeless) part of $a, b, c, \ldots / R$ is a timeless part of one of $a, b, c, \ldots$ or of $R$. 
(R1) is a simple existence condition, and (R3) a fine-grained identity condition. This treats rigid embodiments formed when e.g. $a$ is above $b$ and when $b$ is below $a$ as distinct. Fine considers an alternate coarser-grained identity condition that treats such rigid embodiments as identical as follows.

(R3') Identity': For $e=a, b, c, \ldots / R$ and $e^{\prime}=a^{\prime}, b^{\prime}, c^{\prime}, \ldots / R^{\prime}, e=e^{\prime}$ iff the state of $a, b, c, \ldots$ standing in $R$ is the same as the state of $a^{\prime}, b^{\prime}, c^{\prime}, \ldots$ standing in $R^{\prime}$.

Then a corresponding variant of (R4) is motivated.

(R4') Parthood': $e=a, b, c, \ldots / R$ is a (timeless) part of $e^{\prime}=a^{\prime}, b^{\prime}, c^{\prime}, \ldots / R^{\prime}$ iff the state of $a, b, c, \ldots$ standing in $R$ is part of the state of $a^{\prime}, b^{\prime}, c^{\prime}, \ldots$ standing in $R^{\prime}$.

Of course these two principles rely on an independent understanding of identity and parthood among states, which would require additional theory. For this reason in what follows our focus will be on (R3) and (R4) rather than (R3') and (R4').

(R2) and (R5) play a minimal role in the resulting theory. (R2) secures intuitive locations of rigid embodiments whose components are located; it should be noted that this is compatible with a rigid embodiment existing at $t$ without being located at any point at $t$. (R5) yields the hylomorphic idea that the embodied relation is itself a part of the embodiment. ${ }^{1}$

Fine's theory provides a satisfactory account of the existence conditions of objects like the ham sandwich. He takes the ham sandwich to be a rigid embodiment: its material parts are the two slices of bread and the piece of ham, and its formal part is the betweenness relation. The ham sandwich exists whenever the piece of ham is between the two slices of bread, which seems to appropriately account for the ham sandwich's existence conditions.

Secondly, Fine develops an account of variable embodiments.

Variable Embodiments Given any suitable function $F$ (taking times to things) there is a corresponding object $/ F /$.

Here $/ F /$ is the variable embodiment, $F$ is the principle of variable embodiment, and $/ .$. / denotes some sort of operation of variable embodiment, and the various objects picked out by $F$ are the manifestations of a variable embodiment. Where $f=/ F /, f_{t}$ is $F(t)$ i.e. the manifestation of $f$ at $t$. Variable embodiments satisfy the following principles:

(V1) Existence: $f=/ F /$ exists at $t$ iff $f$ has a manifestation at $t$, i.e. iff $f_{t}$ exists. (V2) Location: If $f=/ F /$ exists at $t$ then $f$ is located (at $t$ ) where $f_{t}$ is located (if at all).

(V3) Identity: $/ F /=/ G /$ iff $F=G$.

\footnotetext{
${ }^{1}$ Fine also wants a range of characterization postulates to do the work of saying how properties of the embodiment are inherited by properties of the parts (either singly, or collectively). It is not clear how these postulates are supposed to be given in a general way, and in any case they aren't given in the theory and so we won't concern ourselves with them.
} 
(V4) Parthood: Any manifestation of a variable embodiment at a given time is a temporary part of it at that time.

(V5) Chaining: If $a$ is a timeless part of $b$ that exists at $t$ and if $b$ is a part of $c$ at $t$, then $a$ is $a$ part of $c$ at $t$. Similarly, if $a$ is part of $b$ at $t$ and if $b$ is a timeless part of an object $c$ that exists at $t$, then $a$ is $a$ part of $c$ at $t$.

(V6) That's All Folks: If $a$ is a temporary part of $b$ at $t$ then there is a mereological chain at $t$ connecting $a$ to $b$.

(V1)-(V4) are obvious correlates to (R1)-(R4). (V3) is a fine-grained identity condition. ${ }^{2}$ There is perhaps space for an even finer-grained identity condition. If principles of variable embodiment are construed as merely having a selecting role, then extensionally equivalent functions result in the same variable embodiment. If principles of variable embodiment are construed as having in addition a characterizing role, then perhaps functions with different conceptual content may generate different variable embodiments. ${ }^{3}$ (V5) is a transitivity condition; it states that any result of chaining together a timeless and temporary part is a temporary part.

For (V6), we define a fundamental link at $t$ to be the result of (V4), namely the temporary parthood of some manifestation to its variable embodiment. (An auxiliary link at $t$ is a case of timeless parthood between entities that exist at $t$.) By a merelogical chain at $t$, Fine means a sequence of links such that at least one of these links is fundamental at $t$.

Note that the 'constituent aspect' analogue of (R5) is not present. Fine writes, 'the principle $F$ stands in a purely external relationship to the manifestation $f_{t}$, whereas the manifestation is actually part of the embodiment.' This is compatible, though, with the principle $F$ standing in an internal relationship to the variable embodiment. On this way of thinking $F$ would be a part of $/ F /$ at every time. This is analogous to the more explicitly hylomorphic rigid embodiments, and something which Fine comes to accept in [13, p. 162]. ${ }^{4}$

Fine's theory offers a satisfactory account of existence conditions for objects like a car. He takes the car to be a variable embodiment, one having as its manifestation at $t$ a rigid embodiment that contains the carburettor as one of its timeless parts. Thus, at $t$, the carburettor is also a temporary part of the car, by (V5). The carburettor is not a part of the rigid embodiment that is a manifestation of the car at a time $t^{\prime}$ at which the carburettor has been replaced by another one. Thus, at $t^{\prime}$, the carburettor is not a temporary part of the car. Moreover, if the car has no manifestation at $t^{\prime \prime}$ (e.g., if at $t^{\prime \prime}$ the car's parts are not assembled), then it is not the case that the carburettor is a timeless part at $t^{\prime \prime}$ of the car.

\footnotetext{
${ }^{2}$ Fine actually puts the condition as follows:

(V3) Identity: $/ F /=/ G /$ iff $F$ and $G$ are the same.
}

We assume what he means by 'sameness' of function is identity, rather than any analogue of 'sameness' in the coarser-grained (R3').

${ }^{3}$ See Evnine [5] for an exploration of this idea.

${ }^{4}$ This additional constraint will be implemented in our semantics; see Definitions 6 and 9. Evnine [5, p. 55 fn 36] claims that Fine (via personal communication) does not regard the principle of variable embodiment as being part of it, though it is not clear why. 


\section{Choice Points}

Fine [10] appears to intend his theory of embodiments to be developed in conjunction with an abundantist view on what properties and relations there are. This fact raises a challenge to any formulation of a formal semantics for the theory of embodiments. On the face of it, this theory is inconsistent with abundantism, as already shown by the Russellian argument in Fairchild [6].

The apparent inconsistency with Cantorian considerations of size also presents itself when considering how to model Fine's hylomorphic theory while taking properties to be abundant. Let $d_{t}$ be the domain of an arbitrary time $t$. That is, $d_{t}$ is a set that represents the collection of objects that exist at $t$. If our models are abundantist, then, for any subset $\mathcal{S}$ of $d_{t}$, there is at least one "property" (i.e., a function from the set $\mathscr{T}$ of "times" to the powerset of $d_{t}$ ) whose extension at $t$ is $\mathcal{S}$. So, for each $\mathcal{S} \subseteq d_{t}$, let:

$C H(\mathcal{S})= \begin{cases}\text { an arbitrary property whose extension at } t \text { is } \mathcal{S} \text { if } \mathcal{S} \text { is nonempty } \\ \text { the "identity" relation } & \text { if } \mathcal{S}=\emptyset\end{cases}$

Also, let:

$\operatorname{ch}(\mathcal{S})=\left\{\begin{array}{l}\text { an arbitrary member of } \mathcal{S} \text { if } \mathcal{S} \text { is nonempty } \\ \text { an arbitrary member of } d_{t} \text { if } \mathcal{S}=\emptyset\end{array}\right.$

In our models there will be some (possibly partial) function em $b(\cdot)$ mapping $n$ ary sequences of elements of $d_{t}$ and $n$-ary relations, to the set-theoretic constructs that represent rigid embodiments. So, let $f$ be a possibly partial function with domain the powerset of $d_{t}$ and such that, for every $\delta \subseteq d_{t}$ :

$$
f(\mathcal{S})= \begin{cases}e m b(\operatorname{ch}(\mathcal{S}), C H(\mathcal{S})) & \text { if } \mathcal{S} \text { is nonempty } \\ e m b(\operatorname{ch}(\mathcal{S}), \operatorname{ch}(\mathcal{S}), C H(\mathcal{S})) & \text { if } \mathcal{S}=\emptyset\end{cases}
$$

Respecting Fine's postulate (R1), requires that, for every $\mathcal{S} \subseteq d_{t}, f(\mathcal{S}) \in$ $d_{t}$. To see this, note that if $\mathcal{S}$ is nonempty, then $\operatorname{ch}(\mathcal{S}) \in \mathcal{S}$. But $\mathcal{S}$ is the extension of $C H(\mathcal{S})$ at $t$. So, $\operatorname{ch}(\mathcal{S})$ has property $C H(\mathcal{S})$ at $t$, in which case emb $(\operatorname{ch}(\mathcal{S}), C H(\mathcal{S})$ ) exists at $t$ by (R1). Similarly, if $\mathcal{S}$ is empty, then $\langle\operatorname{ch}(\mathcal{S}), \operatorname{ch}(\mathcal{S})\rangle$ belongs to the extension of the identity relation at $t$, in which case $e m b(\operatorname{ch}(\mathcal{S}), \operatorname{ch}(\mathcal{S}), C H(\mathcal{S}))$ stands in the identity relation to itself at $t$. Therefore, respecting Fine's postulate $(\mathrm{R} 1)$ requires that $f(\cdot)$ be a function with range $d_{t}$.

Furthermore, respecting postulate (R3) requires that $f$ be a 1-1 function. To see this, suppose that $f\left(\mathcal{S}_{1}\right)=f\left(\mathcal{S}_{2}\right)$. Then, by, (R3), $C H\left(\mathcal{S}_{1}\right)=C H\left(\mathcal{S}_{2}\right)$, and so $\mathcal{S}_{1}=\mathcal{S}_{2}$, on the assumption that properties have only one set as its extension at $t$. So, $f$ is $1-1$. But this is impossible. By Cantor's Theorem, there is no 1-1 function with domain the power set of $d_{t}$ and range $d_{t}$.

So, the formulation of a model theory adequate to Fine's theory requires abandoning an abundantist conception of properties, or else it requires abandoning the idea that for any property $Y$ and individual $x$ instantiating $Y$ there is an embodiment $x / Y$. Our option will be not to have our models incorporate an abundantist conception of properties. Yet, a mitigated form of abundantism will be reflected in the model theory. The principal idea that provides the basis for the model theory is what might be called an iterative conception of wholes. In the model theory objects and relations are relativised to stages. Stage $i$ relations are defined in terms of the set of times and of stage $i$ objects, with the consequence that there are at least as many stage $i$ 
properties as subsets of the set of entities of stage $i$. The objects of stage $i+1$ consist of stage $i$ objects together with new wholes (rigid and variable embodiments) defined in terms of the objects and relations of stage $i$. This iterative conception of wholes somewhat resembles the simultaneous definition of hierarchies of sets and properties formulated in Fine [7, p. 142]. There, the properties of sets of a given level are used to define sets of a higher level, and these sets themselves are then used to define properties not found at previous levels.

The object language first-order quantifiers will range over the objects of any stage $i$ smaller than some limit ordinal $\kappa$. The second-order quantifiers of the object language will range over the relations of any stage $i$ smaller than some limit ordinal $\kappa$. Given the iterative structure exhibited by our models, it would be natural to also add to the language restricted first- and second-order quantifiers, ones indexed to stages. Even though we do not do so here, we note that once such quantifiers are added to the language forms of property comprehension formulated in terms of them could then be formulated. Thus, as already mentioned, the formal semantics to be offered exhibits a minimally abundantist conception of properties, one according to which for any subclass of objects of stage $i$ there is a property of stage $i$ corresponding to it.

One different choice point concerns the interaction between predication and being. The issue concerns whether things may stand in relations at times at which they are nothing. For instance, does Socrates currently have a property even though, arguably, he is now nothing? For simplicity, in the model theory we will define relations in such a way that if things stand in a relation $\mathscr{X}$ at any time $t$, then all of them belong to the domain of $t$. This will make it possible to offer relatively simple characterisations of temporary parthood and of the relation of being mereologically chained.

Yet another choice point concerns the object language expressions for tense. Even though our option has been to use modal operators for this purpose, analogues of the results to be presented would also be available if tense were treated via quantification over times.

\section{A Formal Semantics}

We begin the presentation of the formal semantics for Fine's theory of embodiments by defining a class of frames and other set-theoretic constructs characterised in terms of these.

\subsection{Frames}

Frames are defined as follows:

Definition 1 (Frame) A frame is a quintuple $\mathscr{F}=\left\langle\mathscr{T}, \mathscr{P}, d_{0}, \kappa, \leq\right\rangle$, where:

1. $\mathscr{T}$ and $\mathscr{P}$ are nonempty sets;

2. $d_{0}$ is a function with domain $\mathscr{T} \cup(\mathscr{T} \times \mathscr{P})$ such that:

$\forall t, p: t \in \mathscr{T} \& p \in \mathscr{P}\left(d_{0, t, p} \subseteq d_{0, t}\right)$;

3. $\mathscr{T}, \mathscr{P}$ and $\bigcup_{t \in \mathscr{T}}\left(d_{0, t}\right)$ are pairwise disjoint; 
4. $\kappa$ is a limit ordinal;

5. $\leq$ is a nonstrict total order on $\mathscr{T}$.

Our frames enrich frames for variable-domain first-order temporal logic. As in temporal logic, the set $\mathscr{T}$ represents the set of times and $\leq$ represents the ordering of times (for simplicity, we assume that times are totally ordered).

In variable-domain first-order temporal logic, $d_{0, t}$ represents the set of all individuals that exist at time $t$, for each time $t$. Presently, $d_{0, t}$ does not represent the set of all individuals that exist at $t$. Rather, it represents the set of those individuals that exist at $t$ and have no proper parts. It is natural to understand Fine as endorsing what may be called a 'constructive' or 'iterative' conception of wholes. Wholes are constructed in stages. There is a first stage containing the individuals that have no proper parts. ${ }^{5}$ Here, this first stage will be represented by the set $\mathscr{B}_{0}=\bigcup_{t \in \mathscr{T}} d_{0, t}$. At each stage greater than 0 , new wholes are constructed out of the individuals and the relations that exist at previous stages.

For each stage $i$, the relations of that stage are modelled by functions from times to sets of sequences of individuals of stage $i$ that exist at that time. The wholes of stage $i+1$ are built out of the objects and relations of stage $i$. When $i$ is a limit ordinal, the individuals of stage $i$ are the individuals found at any stage before $i$. The limit ordinal $\kappa$ constitutes a 'cap' on the construction of wholes. The first-order quantifiers of our theory will range over the individuals of this stage.

The set $\mathscr{P}$ has no correspondent in variable-domain first-order temporal logic. Here, this set represents the set of spatial points. ${ }^{6}$ The function $d_{0}$ also takes as arguments time-location pairs. For each time $t$ and location $p, d_{0, t, p}$ represents the set of individuals with no proper parts that exist at $t$ and are located at $p$. It is for this reason that we require that $d_{0, t, p} \subseteq d_{0, t}$.

\subsection{Domains of Individuals and Relations}

We now offer, for each stage, simultaneous definitions of the different domains of entities of that stage. We start by defining the domains of $n$-ary relations. These are defined as usual, to wit, as functions from the set of times to sets of sequences of elements belonging to the domain of that time. That is, for each time $t$, an $n$-ary relation of stage $i$ maps $t$ to a set of $n$-element sequences of elements of $d_{i, t}$ (the set of all individuals of stage $i$ that exist at time $t$ ).

\section{Definition 2 (Domain of stage $i$-ary relations)}

For every $i<\kappa$ and $n \in \mathbb{N}$ :

\footnotetext{
${ }^{5}$ We needn't see these 'atoms' as purely partless; the approach outlined here is compatible with treating them as (pieces of) gunk, whose structure is taken for granted for modelling purposes. Or better, this framework could be supplemented with Fine's Segmentation approach to decomposing structural atoms $[14$, p. 578]. In this paper, we are attempting to model how the structural parts of embodiments behave; segmentation could usefully be employed to model how spatial parts of these objects behave.

${ }^{6} \mathrm{We}$ impose no structure on this the set of spatial points. Thus, applications of the semantics may require the exclusion of some frames as representationally insignificant with respect to the structure of space.
} 
1. A stage $i n$-ary relation is a function $\mathscr{X}$ such that:

$$
\mathscr{X} \in\left(\wp\left(\left(\bigcup_{t \in \mathscr{T}}\left(d_{i, t}\right)\right)^{n}\right)\right)^{\mathscr{T}} \text { and } \forall t \in \mathscr{T}\left(\mathscr{X}(t) \subseteq\left(d_{i, t}\right)^{n}\right){ }^{7}
$$

2. $\mathscr{D}_{i}^{n}$ is the set of stage $i n$-ary relations;

3. $\mathscr{D}_{\kappa}^{+}=\bigcup_{n \in \mathbb{Z}^{+}} \bigcup_{j<\kappa} \mathscr{D}_{j}^{n}$ is the set of all relations of positive arity.

4. $\mathscr{D}_{\kappa}=\bigcup_{n \in \mathbb{N}} \bigcup_{j<\kappa} \mathscr{D}_{j}^{n}$ is the set of all relations of any arity.

Note that Definition 2 requires that individuals stand in a relation at a time only if they all exist at that time. As mentioned in Section 2, our models will all incorporate this simplifying assumption.

We now turn to the definition of the set of embodiments of stage $i$.

\section{Definition 3 (Stage $i$-ary embodiments)}

For every ordinal $i$ such that $0<i \leq \kappa$ and $n \in \mathbb{Z}^{+}$, an $n$-ary embodiment of stage $i$ is an $n+1$-tuple $e=\left\langle m_{1}, \ldots, m_{n}, \mathscr{X}\right\rangle$ such that:

1. If $i=\alpha+1$, then $\forall j\left(1 \leq j \leq n \Rightarrow m_{j}: \mathscr{T} \rightarrow \bigcup_{t \in \mathscr{T}}\left(d_{\alpha, t}\right)\right),{ }^{8}$ and $\mathscr{X} \in \mathscr{D}_{\alpha}^{n}$.

2. If $i$ is a limit ordinal, then $e$ is a stage $j n$-ary embodiment, for some $j<i$.

According to Definition 3, an $n$-ary embodiment of stage $i+1$ is an $n+1$-tuple whose first $n$ members are partial functions from times to objects of at most stage $i$ and whose $(n+1)^{\text {th }}$ member is an $n$-ary relation between objects of at most stage $\alpha$. For each embodiment $e=\left\langle m_{1}, \ldots, m_{n}, \mathscr{X}\right\rangle$ and time $t$, each member $m_{i}$ of $e$ determines a "material part" $m_{i}(t)$ of $e$ at $t$. The function $\mathscr{X}$ is the "principle of embodiment" of $e$.

We now define rigid embodiments as special kinds of embodiments:

\section{Definition 4 (Stage $i n$-ary rigid embodiments)}

For every $0<i \leq \kappa$ and $n \in \mathbb{Z}^{+}$:

1. A stage $i n$-ary rigid embodiment is a stage $i n$-ary embodiment $e=$ $\left\langle m_{1}, \ldots, m_{n}, \mathscr{X}\right\rangle$ such that, for every $j$ such that $1 \leq j \leq n$ :

(a) $\forall t \in \mathscr{T} \exists x\left(x=m_{j}(t)\right)$; and

(b) $\forall t, t^{\prime} \in \mathscr{T}\left(m_{j}(t)=m_{j}\left(t^{\prime}\right)\right)$

2. $\mathscr{R}_{i}^{n}=\bigcup_{j \leq i}\{e: e$ is a stage $j n$-ary rigid embodiment $\}$ is the set of $n$-ary rigid embodiments of at most stage $i$;

3. $\mathscr{R}_{\kappa}^{n}=\bigcup_{i<\kappa} \mathscr{R}_{i}^{n}$ is the set of all $n$-ary rigid embodiments;

${ }^{7}$ Where:

- $\left(\bigcup_{t \in \mathscr{T}}\left(d_{i, t}\right)\right)^{n}=\underbrace{\bigcup_{t \in \mathscr{T}}\left(d_{i, t}\right) \times \ldots \times \bigcup_{t \in \mathscr{T}}\left(d_{i, t}\right)}_{n \text { times }}$;

- $\wp\left(\left(\bigcup_{t \in \mathscr{T}}\left(d_{i, t}\right)\right)^{n}\right)$ is the powerset of $\left(\bigcup_{t \in \mathscr{T}}\left(d_{i, t}\right)\right)^{n}$; and

- $\quad\left(\wp\left(\left(\bigcup_{t \in \mathscr{T}}\left(d_{i, t}\right)\right)^{n}\right)\right)^{\mathscr{T}}$ is the set of all functions from $\mathscr{T}$ to $\wp\left(\left(\bigcup_{t \in \mathscr{T}}\left(d_{i, t}\right)\right)^{n}\right)$.

${ }^{8}$ That is, $m_{j}$ is a partial function with domain $\mathscr{T}$ and codomain $\mathscr{B}_{\alpha}$. 
4. $\mathscr{R}_{i}=\bigcup_{n \in \mathbb{Z}^{+}} \mathscr{R}_{i}^{n}$ is the set of rigid embodiments (of some arity) of at most stage $i$;

5. $\mathscr{R}_{\kappa}=\bigcup_{i<\kappa} \mathscr{R}_{i}$ is the set of all rigid embodiments.

According to Definition 4, a rigid embodiment is an embodiment that contains the same material parts at every time. The definition of variable embodiments will appeal to a special type of property, defined as follows:

\section{Definition 5 (Stage $i$ individual concept)}

For every $i<\kappa$ :

1. A stage $i$ individual concept is a function $\mathscr{X} \in \mathscr{D}_{i}^{1}$ such that:

(a) $\forall t \in \mathscr{T}(|\mathscr{X}(t)|=1$ or $\mathscr{X}(t)=\emptyset)$; and

(b) $\exists t, t^{\prime} \in \mathscr{T}\left(|\mathscr{X}(t)|=\left|\mathscr{X}\left(t^{\prime}\right)\right|=1 \& \mathscr{X}(t) \neq \mathscr{X}\left(t^{\prime}\right)\right)$.

2. $\mathscr{I}_{i}$ is the set of stage $i$ individual concepts.

According to Definition 5, $\mathscr{X}$ is an individual concept just in case: (i) $\mathscr{X}$ is instantiated by at most one individual at each moment in time; and (ii) there are times $t$ and $t^{\prime}$ such that $\mathscr{X}$ is instantiated both at $t$ and $t^{\prime}$, and the individual instantiating $\mathscr{X}$ at $t$ is distinct from the individual instantiating $\mathscr{X}$ at $t^{\prime}$. We are now in a position to define variable embodiments:

\section{Definition 6 (Stage $i$ variable embodiments)}

For every $0<i \leq \kappa$ :

1. A stage $i$ variable embodiment is a stage $i$ unary embodiment $e=\langle m, \mathscr{X}\rangle$ such that:

(a) $\mathscr{X}$ is a stage $i$ individual concept; and

(b) $\forall t \in \mathscr{T}:$

(i) $\forall x(\mathscr{X}(t)=\{x\} \Rightarrow m(t)=x)$; and

(ii) $\neg \exists x(\mathscr{X}(t)=\{x\}) \Rightarrow \neg \exists x(m(t)=x)$.

2. $\mathscr{V}_{i}=\bigcup_{j \leq i}\{e: e$ is a stage $j$ variable embodiment $\}$ is the set of variable embodiments of at most stage $i$;

3. $\mathscr{V}_{\kappa}=\bigcup_{i<\kappa} \mathscr{V}_{i}$ is the set of all variable embodiments.

Whereas in the case of rigid embodiments, each partial function $m_{j}$, for each $j$ such that $1 \leq j \leq n$, determines the same individual with respect to every time, in the case of variable embodiments each partial function $m$ determines possibly different individuals at different times. Moreover, in the case of variable embodiments the partial function $m$ is determined in terms of the individual concept $\mathscr{X}$. For each time $t, m(t)$ is the unique individual falling under $\mathscr{X}$ at $t$, if there is such an individual, and otherwise $m(t)$ is undefined. 
Prima facie, $n$-ary rigid embodiments would more naturally be represented as $n$ tuples of objects (rather than functions from times to objects) and $n$-ary relations. The advantage of our representation of embodiments is that it makes possible for a representation of both rigid and variable embodiments as entities of the same kind. As we shall see, it also enables a unifying representation of the notion of immediate parthood at a time.

We now define the set of individuals of at most stage $i$ that exist at time $t$ :

\section{Definition 7 (Domain of individuals of at most stage $i$ that exist at $t$ )}

For every $i \leq \kappa$ and $t \in T$ :

1. $x$ is an individual of at most stage $i$ that exists at $t$ if and only if either:

(a) $x \in d_{0, t}$, if $i=0$;

(b) there is some $n \in \mathbb{Z}^{+}$and $j \leq i$ such that $x=\left\langle m_{1}, \ldots, m_{n}, \mathscr{X}\right\rangle \in$ $\left(\mathscr{R}_{j}^{n} \cup \mathscr{V}_{j}\right)$ and

$\exists x_{1}, \ldots x_{n}\left(x_{1}=m_{1}(t) \& \ldots \& x_{n}=m_{n}(t) \&\left\langle x_{1}, \ldots, x_{n}\right\rangle \in\right.$ $\mathscr{X}(t))$, if $i>0$.

2. $d_{i, t}$ is the set of stage $i$ individuals of $t$;

3. $d_{\kappa, t}=\bigcup_{i<\kappa} d_{i, t}$ is the set of all individuals of $t$;

4. $\mathscr{B}_{i}=\bigcup_{t \in \mathscr{T}} d_{i, t}$ is the set of individuals of at most stage $i$ that exist at some time;

5. $\mathscr{B}_{\kappa}=\bigcup_{i<\kappa} \mathscr{B}_{i}$ is the set of all individuals that exist at some time.

According to Definition 7, an individual of at most stage $i$ that exists at time $t$ is either an element of $d_{0, t}$, if $i=0$, or else it is a rigid or variable embodiment of at most stage $i$ whose material parts at $t$ stand, at $t$, in the relation that is the individual's principle of embodiment.

The set of stage $i$ individuals of $t$ is a superset of the set of stage $i$ individuals of $t$ that are located at $p$ :

\section{Definition 8 (Domain of at most stage $i$ individuals of $t$ that are located at $p$ )}

For every $i \leq \kappa, t \in T$ and $p \in P$ :

1. A stage $i$ individual $x$ of $t$ located at $p$ is either:

(a) An element of $d_{0, t, p}$, if $i=0$; or else

(b) there is some $n \in \mathbb{Z}^{+}$such that $x=\left\langle m_{1}, \ldots, m_{n}, \mathscr{X}\right\rangle \in d_{i, t} \& \exists l, j(1 \leq$ $\left.l \leq n \& j<i \& m_{l}(t) \in d_{j, t, p}\right)$, if $i>0$.

2. $d_{i, t, p}$ is the set of at most stage $i$ individuals of $t$ that are located at $p$;

3. $d_{\kappa, t, p}$ is the set of all individuals of $t$ that are located at $p$.

According to Definition 8, an embodiment is located at a point $p$ and time $t$ just in case some of its material parts at $t$ are located at point $p$ and time $t$. 


\subsection{Mereological Notions}

We now turn to the characterisation of mereological notions in terms of frames. These will be the interpretations of the mereological predicates of our language.

The most basic notion is that of immediate parthood at $t$ :

\section{Definition 9 (Immediate parthood)}

For every frame $\mathscr{F}, t \in \mathscr{T}, n \in \mathbb{N}$, and $x, y, x$ is an immediate part of $y$ at $t$ (and $\mathscr{F}), x \ll_{\mathscr{F}}^{t} y$ if and only if there is an $n \in \mathbb{Z}^{+}$such that

1. $y=\left\langle m_{1}, \ldots, m_{n}, \mathscr{X}\right\rangle \in d_{\kappa, t} \cap\left(\mathscr{R}_{\kappa}^{n} \cup \mathscr{V}_{\kappa}\right)$; and

2. either (i) $\exists i: 1 \leq i \leq n\left(x=m_{i}(t) \& x \in d_{\kappa, t}\right)$, or (ii) $x=\mathscr{X}$.

Thus, according to Definition $9, x$ is an immediate part of $y$ at $t$ just in case $y$ exists at $t$ and $x$ is either a material part of $y$ at $t$ that exists at $t$ or else $x$ is $y$ 's principle of embodiment. The definition of parthood appeals to the following definition:

\section{Definition 10 (Immediate Parthood Sequence)}

For every frame $\mathscr{F}$, time $t \in \mathscr{T}, n \in \mathbb{Z}^{+}$and sequence of objects $\vec{x}_{n}=$ $\left\langle x_{1}, \ldots, x_{n}\right\rangle, \vec{x}_{n}$ is an $n$-element immediate parthood sequence at $t$ (and $\mathscr{F}$ ), $\ll_{\mathscr{F}}^{t}\left(\vec{x}_{n}\right)$, if and only if $n \geq 2$ and $\forall i: 1 \leq i<n\left(x_{i} \ll_{\mathscr{F}}^{t} x_{i+1}\right)$.

According to Definition 10, an immediate parthood sequence is a sequence each member of which is an immediate part of its successor, whenever it has a successor. Proper parthood is defined as follows:

\section{Definition 11 (Proper Parthood)}

For every frame $\mathscr{F}$, time $t \in \mathscr{T}$ and $x, y, x$ is a proper part of $y$ at $t$ (and frame $\mathscr{F}), x<_{\mathscr{F}}^{t} y$, if and only if:

$\exists n \in \mathbb{Z}^{+} \exists \vec{x}_{n}\left(\ll_{\mathscr{F}}^{t}\left(\vec{x}_{n}\right) \& x=x_{1} \& y=x_{n}\right)$.

According to Definition 11, to be a proper part is to be a member of a chain of immediate parthood. Parthood is defined in the usual manner:

\section{Definition 12 (Parthood)}

For every frame $\mathscr{F}$, time $t \in \mathscr{T}$ and $x, y, x$ is a part of $y$ at $t$ (and $\mathscr{F}$ ), $x \leqslant_{\mathscr{F}}^{t} y$, if and only if:

$$
x<_{\mathscr{F}}^{t} y \text { or } x=y \in d_{\kappa, t} \cup \mathscr{D}_{\kappa}^{+} \text {. }
$$

We now turn to the definition of proper timeless parthood:

\section{Definition 13 (Proper Timeless Parthood)}

For every frame $\mathscr{F}$, time $t \in \mathscr{T}$ and $x, y, x$ is a proper timeless part of $y$ at $t$ (and $\mathscr{F}$ ), $x \triangleleft_{\mathscr{F}}^{t} y$, if and only if: 
$\exists n \in \mathbb{Z}^{+} \exists \vec{x}_{n}\left(\ll_{\mathscr{F}}^{t}\left(\vec{x}_{n}\right) \& x=x_{1} \& y=x_{n} \& \forall i: 1 \leq i<n\left(x_{i+1} \in\right.\right.$ $\mathscr{R}_{\kappa}^{n}$ or $\left.\left.x_{i} \in \mathscr{D}_{\kappa}^{+}\right)\right)$.

According to Definition $13, x$ is a proper timeless part of $y$ just in case there is an immediate parthood sequence linking $x$ to $y$ whose members are all rigid embodiments except, perhaps, $x$ or else $x$ is a variable embodiment, its successor in the immediate parthood sequence is a variable embodiment, and all other elements in the immediate parthood sequence linking $x$ to $y$ are rigid embodiments.

\section{Definition 14 (Timeless parthood)}

For every frame $\mathscr{F}$, time $t \in \mathscr{T}$ and $x, y, x$ is a timeless part of $y$ at $t$ (and $\mathscr{F}$ ), $x \unlhd_{\mathscr{F}}^{t} y$, if and only if:

$$
x \triangleleft_{\mathscr{F}}^{t} y \text { or } x=y \in d_{\kappa, t} \cup \mathscr{D}_{\kappa}^{+} \text {. }
$$

Besides timeless parthood, the other mereological primitives of Fine's theory are the relations of temporary parthood, of being a manifestation, and of being mereologically chained. Manifestation is defined as follows:

\section{Definition 15 (Manifestation)}

For every frame $\mathscr{F}$, time $t \in \mathscr{T}$ and $x, y, x$ is a manifestation of $y$ at $t$ (and $\mathscr{F}$ ), $x M_{\mathscr{F}}^{t} y$, if and only if:

$y \in d_{\kappa, t} \cap \mathscr{V}_{\kappa}$ and $\exists m, \mathscr{X}\left(y=\langle m, \mathscr{X}\rangle \& x=m(t) \in d_{\kappa, t}\right)$.

According to Definition 15 , for $x$ to be a manifestation of an entity $y$ is for $y$ to be variable embodiment and for $x$ to be $y$ 's material part at $t$. Proper temporary parthood is defined in terms of the mereological notions of parthood and manifestation as follows:

\section{Definition 16 (Proper Temporary parthood)}

For every frame $\mathscr{F}$, time $t \in \mathscr{T}$ and $x, y, x$ is a proper temporary part of $y$ at $t$ (and $\mathscr{F}$ ), $x \prec_{\mathscr{F}}^{t} y$, if and only if:

$$
\exists u, \succsim \in d_{\kappa, t}\left(x \leqslant_{\mathscr{F}}^{t} u \& u M_{\mathscr{F}}^{t} z \& \approx \leqslant_{\mathscr{F}}^{t} y\right) .
$$

According to Definition 16 , to be a proper temporary part of $x$ is to have as a part a variable embodiment whose manifestation has $x$ among its parts. Temporary parthood is defined as expected:

\section{Definition 17 (Temporary parthood)}

For every frame $\mathscr{F}$, time $t \in \mathscr{T}$ and $x, y, x$ is a temporary part of $y$ at $t$ (and $\mathscr{F}$ ), $x \preceq_{\mathscr{F}}^{t} y$, if and only if:

$$
x \prec_{\mathscr{F}}^{t} y \text { or } x=y \in d_{\kappa, t} \cup \mathscr{D}_{\kappa}^{+} \text {. }
$$

We now define the notion of a mereological chain:

\section{Definition 18 (Mereological chain)}

For every frame $\mathscr{F}$, time $t \in \mathscr{T}, n \in \mathbb{Z}^{+}$and $n$-ary sequence $\vec{x}_{n}=\left\langle x_{1}, \ldots, x_{n}\right\rangle$, $\vec{x}_{n}$ is a mereological chain at $t$ (and $\left.\mathscr{F}\right), \rightsquigarrow_{\mathscr{F}}^{t}\left(\vec{x}_{n}\right)$, if and only if: 
$n \geq 2 \& \forall i: 1 \leq i<n\left(x_{i} M_{\mathscr{F}}^{t} x_{i+1}\right.$ or $\left.x_{i} \triangleleft_{\mathscr{F}}^{t} x_{i+1}\right) \& \exists i: 1 \leq i<$ $n\left(x_{i} M_{\mathscr{F}}^{t} x_{i+1}\right)$.

We can now define the final mereological notion in terms of which Fine's theory is formulated:

\section{Definition 19 (Mereologically chained)}

For every frame $\mathscr{F}$, time $t \in \mathscr{T}$ and $x, y, x$ is mereologically chained to $y$ at $t$ (and $\mathscr{F}$ ), $x \rightsquigarrow_{\mathscr{F}}^{t} y$, if and only if:

$\exists n \in \mathbb{Z}^{+}\left(\exists \vec{x}_{n}\left(\rightsquigarrow_{F}^{t}\left(\vec{x}_{n}\right) \& x_{1}=x \& x_{n}=y\right)\right.$.

Before concluding the characterisation of the language's mereological notions we state a result to the effect that for $x$ to be a temporary part of $y$ at $t$ just is for $x$ to be mereologically chained to $y$ at $t$ :

Lemma 1 For every frame $\mathscr{F}$, time $t \in \mathscr{T}$ and $x, y$ :

$$
x \prec_{\mathscr{F}}^{t} y \text { iff } x \rightsquigarrow \stackrel{F}{F}^{t} \text {. }
$$

\section{Proof of Lemma 1}

$(\Rightarrow)$ : Suppose that $x \prec_{\mathscr{F}}^{t} y$. By Definitions 12 and 17, it follows that there is an immediate parthood sequence at $t$ between $x$ and some $\%$ such that $\%$ is a manifestation at $t$ of some $w$ such that there is an immediate parthood sequence at $t$ between $w$ and $y$. Furthermore, note that, for every $\approx$ and $w$, if $\approx$ is an immediate part at $t$ of $w$, then either $\approx$ is a timeless part of $w$ at $t$, or else $\approx$ is a manifestation at $t$ of $w$, by Definitions 7, 9, 14 and 15. But then, $x \rightsquigarrow \stackrel{t}{\mathscr{F}} y$.

$(\Leftarrow)$ : Clearly, for any $\%$ and $w$, if $\%$ is a manifestation of $w$ at $t$, then $\%$ is an immediate part of $w$ at $t$, and if $z$ is a timeless part of $w$ at $t$ then $\%$ is an immediate part of $w$ at $t$. Either way, $z$ is an immediate part of $w$ at $t$. Now, suppose that $x \rightsquigarrow t_{\mathscr{F}}^{t} y$. It follows from the above and Definition 19 that there is an immediate parthood sequence at $t$ linking $x$ to some element $\approx$ that is a manifestation at $t$ of some element $w$ which is such that there is an immediate parthood sequence at $t$ linking $w$ to $y$. So, $x \prec_{\mathscr{F}}^{t} y$.

This result concludes the characterisation of our target frames. In what follows we define a language with sufficient resources for the formulation of Fine's theory of embodiments.

\subsection{Language}

Fine's theory of embodiments will be formulated in the language $\mathrm{L}_{\mathrm{E}}$, a language obtained from a second-order language by adding: (i) variables for spatial points, (ii) the temporal operators ' $H$ ' ('it was always that') and ' $G$ ' ('it will always be that'); (iii) the location predicate $\ulcorner\operatorname{Loc}(s)\urcorner$, for every spatial variable $s$ (where ' $\operatorname{Loc}(p)$ ' stands for a predicate standing the property of being located at $p$ ); (iv) '/' (standing for a function mapping an $n$-ary sequence of individuals and an $n$-ary relation to the 
rigid embodiment containing those individuals and that relation as immediate parts) and '/ /' (standing for a function mapping a monadic property to the variable embodiment containing this monadic property as its formal part); (v) the identity predicate '=', flanked either by individual terms or $n$-ary second-order terms; (vi) the mereological binary predicates ' $\ll$ ' (standing for the immediate parthood relation), ' $<$ ' (standing for the proper parthood relation), ' $\triangleleft$ ' (standing for timeless proper parthood), ' $\prec$ ' (standing for temporary proper parthood), ' $\rightsquigarrow$ ' (standing for the relation that obtains between $x$ and $y$ when there is a mereological chain linking $x$ to $y$ ), and ' $M$ ' (standing for the 'is a manifestation of' relation).

In what follows we offer a more precise definition of $\mathrm{L}_{\mathrm{E}}$. We start by defining the language's individual constants, predicate letters and variables:

\section{Definition 20 (Individual constants and predicates of $\mathrm{L}_{\mathrm{E}}$ )}

1. The set $\left\{\left\ulcorner a_{i}\right\urcorner: i \in \mathbb{N}\right\}$ is the set of individual constants of $\mathrm{L}_{\mathrm{E}}$;

2. For each $n \in \mathbb{N}$, the set $\left\{\left\ulcorner R_{i}^{n}\right\urcorner: i \in \mathbb{N}\right\}$ is the set of (uninterpreted) $n$-ary predicates of $\mathrm{L}_{\mathrm{E}}$.

\section{Definition 21 (Variables of $\mathrm{L}_{\mathrm{E}}$ )}

1. $\left\{\left\ulcorner u_{i}\right\urcorner,\left\ulcorner v_{i}\right\urcorner,\left\ulcorner w_{i}\right\urcorner,\left\ulcorner x_{i}\right\urcorner,\left\ulcorner y_{i}\right\urcorner,\left\ulcorner z_{i}\right\urcorner: i \in \mathbb{Z}^{+}\right\}$is the set of individual variables of $\mathrm{L}_{\mathrm{E}}$;

2. For each $n \in \mathbb{N},\left\{\left\ulcorner U_{i}^{n}\right\urcorner,\left\ulcorner V_{i}^{n}\right\urcorner,\left\ulcorner W_{i}^{n}\right\urcorner,\left\ulcorner X_{i}^{n}\right\urcorner,\left\ulcorner Y_{i}^{n}\right\urcorner,\left\ulcorner Z_{i}^{n}\right\urcorner: i \in \mathbb{Z}^{+}\right\}$is the set of $n$-ary second-order variables of $\mathrm{L}_{\mathrm{E}}$;

3. $\left\{\left\ulcorner p_{i}\right\urcorner: i \in \mathbb{Z}^{+}\right\}$is the set of spatial variables of $\mathrm{L}_{\mathrm{E}}$.

The set of $n$-ary second-order terms of $\mathrm{L}_{\mathrm{E}}$ is defined as follows:

\section{Definition 22 ( $n$-ary second-order terms of $\mathrm{L}_{\mathrm{E}}$ )}

1. Every $n$-ary predicate letter is an $n$-ary second-order term;

2. Every $n$-ary second-order variable is an $n$-ary second-order term;

3. Nothing else is an $n$-ary second-order term.

The set of individual terms of $\mathrm{L}_{\mathrm{E}}$ is defined as follows:

\section{Definition 23 (Individual terms of $L_{E}$ )}

1. Every individual variable is an individual term;

2. Every individual constant is an individual term;

3. If $\sigma_{1}, \ldots, \sigma_{n}$ are individual terms and $\zeta$ is an $n$-ary second-order term, then $\left\ulcorner\sigma_{1}, \ldots, \sigma_{n} / \zeta\right\urcorner$ is an individual term;

4. If $\zeta$ is a monadic second-order term, then $\ulcorner/ \zeta /\urcorner$ is an individual term;

5. Nothing else is an individual term.

The set of well-formed formulas (wffs) is defined as follows:

Definition 24 (Well-formed formulae of $\mathrm{L}_{\mathrm{E}}$ ) If $\sigma, \sigma_{1}, \sigma_{2}, \ldots, \sigma_{n}$ are individual terms, $\zeta, \zeta_{1}, \zeta_{2}$ are $n$-ary second-order terms, $\rho_{1}$ and $\rho_{2}$ are either individual terms of 
second-order terms of some arity, $\varphi$ and $\psi$ are wffs, $v$ is an individual variable, $V$ is an $n$-ary second-order variable and $s$ is a spatial variable, then:

1. $\left\ulcorner\zeta \sigma_{1}, \ldots, \sigma_{n}\right\urcorner$ is a wff;

2. $\ulcorner\operatorname{Loc}(s) \sigma\urcorner$ is a wff;

3. $\left\ulcorner\sigma_{1} M \sigma_{2}\right\urcorner$ is a wff;

4. $\left\ulcorner\sigma_{1}=\sigma_{2}\right\urcorner$ is a wff;

5. $\left\ulcorner\zeta_{1}=\zeta_{2}\right\urcorner$ is a wff;

6. $\left\ulcorner\rho_{1} \ll \rho_{2}\right\urcorner$ is a wff;

7. $\left\ulcorner\rho_{1}<\rho_{2}\right\urcorner$ is a wff;

8. $\left\ulcorner\rho_{1} \triangleleft \rho_{2}\right\urcorner$ is a wff;

9. $\left\ulcorner\rho_{1} \prec \rho_{2}\right\urcorner$ is a wff;
10. $\left\ulcorner\rho_{1} \rightsquigarrow \rho_{2}\right\urcorner$ is a wff;

11. $\ulcorner\neg \varphi\urcorner$ is a wff;

12. $\ulcorner\varphi \wedge \psi\urcorner$ is a wff;

13. $\ulcorner\forall v \varphi\urcorner$ is a wff;

14. $\ulcorner\forall V \varphi\urcorner$ is a wff;

15. $\ulcorner\forall s \varphi\urcorner$ is a wff;

16. $\ulcorner H \varphi\urcorner$ is a wff;

17. $\ulcorner G \varphi\urcorner$ is a wff;

Furthermore, nothing else is a wff.

This concludes the definition of the language $\mathrm{L}_{\mathrm{E}}$.

\subsection{Models}

We now define models for $\mathrm{L}_{\mathrm{E}}$ :

\section{Definition 25 (Models)}

A model for $\mathrm{L}_{\mathrm{E}}$ based on a frame $\mathscr{F}=\left\langle\mathscr{T}, \mathscr{P}, d_{0}, \kappa, \leq\right\rangle$ is any sequence $\mathscr{M}=$ $\left\langle\mathscr{T}, \mathscr{P}, d_{0}, \kappa, \leq, v\right\rangle$, where:

1. $v(\sigma) \in \mathscr{B}_{\kappa}$, for each individual constant $\sigma$ of $\mathrm{L}_{\mathrm{E}}$;

2. $v(\zeta) \in \mathscr{D}_{\kappa}^{n}$, for each $n$-ary predicate $\zeta$ of $\mathrm{L}_{\mathrm{E}}$ and $n \in \mathbb{N}$.

\section{Definition 26 (Variable-assignment)}

For every frame $\mathscr{F}$,

1. A variable-assignment based on $\mathscr{F}$ is a function $\mathcal{g}$ such that, for each first-order variable $v, n$-ary second-order variable $V$, and spatial variable $s, q(v) \in \mathscr{B}_{\kappa}$, $\mathscr{g}(V) \in \mathscr{D}_{\kappa}^{n}$ and $\mathscr{g}(s) \in \mathscr{P}$.

2. $A s_{\mathscr{F}}$ is the set of all variable-assignments based on $\mathscr{F}$;

3. Where $g$ is a variable-assignment, $\mathscr{g}[\eta / x]$ is a variable assignment just like $\mathscr{g}$ except that it assigns $x$ to the variable $\eta$ (where $\eta$ and $x$ are such as to respect the conditions required for $q[\eta / x]$ to belong to $A s \mathscr{F}$ ).

We now define the notion of value relative to a variable-assignment and a time:

\section{Definition 27 (Value relative to a variable-assignment and a time)}

1. $v^{q}(\sigma)=\left\{\begin{array}{l}\vartheta(\sigma) \text { if } \sigma \text { is an individual constant } \\ g(\sigma) \text { if } \sigma \text { is a first-order variable }\end{array}\right.$

2. $v^{q}(\zeta)= \begin{cases}v(\zeta) & \text { if } \zeta \text { is an } n \text {-ary predicate letter } \\ g(\zeta) & \text { if } \zeta \text { is an } n \text {-ary second-order variable }\end{cases}$ 
3. $v^{g}\left(\sigma_{1}, \ldots, \sigma_{n} / \zeta\right)= \begin{cases}e=\left\langle\lambda t \in \mathscr{T} \cdot v^{q}\left(\sigma_{1}\right), \ldots, \lambda t \in \mathscr{T} \cdot v^{q}\left(\sigma_{n}\right), v^{q}(\zeta)\right\rangle & \text { if } e \in \mathscr{R}_{\kappa}^{n} \cap \mathscr{B}_{\kappa} \\ \text { undefined } & \text { Otherwise }\end{cases}$

4. $v^{g}(/ \zeta /)= \begin{cases}e=\langle m, \mathscr{X}\rangle \text { such that } \mathscr{X}=v^{\mathcal{g}}(\zeta) & \text { if } e \in \mathscr{V}_{\kappa} \cap \mathscr{B}_{\kappa} \\ \text { undefined } & \text { Otherwise }\end{cases}$

Satisfaction is defined as follows:

Definition 28 (Satisfaction) For every model $\mathscr{M}$ (based on some frame $\mathscr{F}$ ), $t \in \mathscr{T}$ and $\mathscr{q} \in A s_{\mathscr{F}}$ :

1. $M, t, g \vDash \zeta \sigma_{1} \ldots \sigma_{n}$ iff $\forall i: 1 \leq i \leq n\left(\mho^{q}\left(\sigma_{i}\right)\right.$ is defined), and $\left\langle v^{q}\left(\sigma_{1}\right), \ldots, v^{q}\left(\sigma_{n}\right)\right\rangle \in v^{q}(\zeta)(t)$

2. $M, t, q \vDash \operatorname{Loc}(s) \sigma$ iff $\vartheta^{q}(\sigma)$ is defined and $\vartheta^{q}(\sigma) \in d_{\kappa, t, q(s)}$;

3. $M, t, q \vDash \sigma_{1} M \sigma_{2}$ iff $\mho^{q}\left(\sigma_{1}\right)$ and $v^{g}\left(\sigma_{2}\right)$ are defined and $v^{q}\left(\sigma_{1}\right) M_{\mathscr{F}}^{t} v^{q}\left(\sigma_{2}\right)$;

4. $\mathscr{M}, t, q \vDash \sigma_{1}=\sigma_{2}$ iff $v^{q}\left(\sigma_{1}\right) \in d_{\kappa, t}$ and $u^{q}\left(\sigma_{1}\right)=u^{q}\left(\sigma_{2}\right)$;

5. $\mathscr{M}, t, q \vDash \zeta_{1}=\zeta_{2}$ iff $u^{q}\left(\zeta_{1}\right)=v^{q}\left(\zeta_{2}\right)$;

6. $\mathscr{M}, t, \mathcal{g} \vDash \rho_{1} \ll \rho_{2}$ iff $v^{q}\left(\rho_{1}\right)$ and $v^{q}\left(\rho_{2}\right)$ are defined and $v^{q}\left(\rho_{1}\right) \ll_{\mathscr{F}}^{t}$ $v^{q}\left(\rho_{2}\right)$

7. $\mathscr{M}, t, \mathcal{Q} \vDash \rho_{1}<\rho_{2}$ iff $\vartheta^{q}\left(\rho_{1}\right)$ and $v^{q}\left(\rho_{2}\right)$ are defined and $v^{q}\left(\rho_{1}\right)<_{\mathscr{F}}^{t}$ $v^{q}\left(\rho_{2}\right)$;

8. $\mathscr{M}, t, \mathcal{L} \vDash \rho_{1} \triangleleft \rho_{2}$ iff $\vartheta^{q}\left(\rho_{1}\right)$ and $v^{q}\left(\rho_{2}\right)$ are defined and $v^{q}\left(\rho_{1}\right) \triangleleft_{\mathscr{F}}^{t} v^{q}\left(\rho_{2}\right)$;

9. $\mathscr{M}, t, \mathscr{Q} \vDash \rho_{1} \prec \rho_{2}$ iff $\vartheta^{\mathscr{q}}\left(\rho_{1}\right)$ and $v^{q}\left(\rho_{2}\right)$ are defined and $v^{\mathscr{q}}\left(\rho_{1}\right) \prec_{\mathscr{F}}^{t}$ $v^{2}\left(\rho_{2}\right)$;

10. $\mathscr{M}, t, q \vDash \rho_{1} \rightsquigarrow \rho_{2}$ iff $\vartheta^{q}\left(\rho_{1}\right)$ and $\vartheta^{q}\left(\rho_{2}\right)$ are defined and $\vartheta^{q}\left(\rho_{1}\right) \rightsquigarrow \mathscr{F}$ $v^{2}\left(\rho_{2}\right)$;

11. $\mathscr{M}, t, \mathscr{q} \vDash \neg \varphi$ iff $\mathscr{M}, t, \mathscr{q} \not \models$;

12. $\mathscr{M}, t, \mathscr{g} \vDash \varphi \wedge \psi$ iff $\mathscr{M}, t, \mathscr{q} \vDash \varphi$ and $\mathscr{M}, t, \mathscr{g} \vDash \psi$;

13. $\mathscr{M}, t, \mathscr{g} \vDash \forall v \varphi$ iff for all $x \in d_{\kappa, t}: \mathscr{M}, t, \mathscr{q}[v / x] \vDash \varphi$;

14. $\mathscr{M}, t, \mathscr{Q} \vDash \forall V \varphi$ iff for all $\mathscr{X} \in \mathscr{D}_{\kappa}^{n}: \mathscr{M}, t, \mathscr{Q}[V / \mathscr{X}] \vDash \varphi$;

15. $\mathscr{M}, t, q \vDash \forall s \varphi$ iff for all $p \in \mathscr{P}: \mathscr{M}, t, q[s / p] \vDash \varphi$;

16. $\mathscr{M}, t, \mathscr{g} \vDash H \varphi$ iff for all $t^{\prime} \in \mathscr{T}$ s. t. $t^{\prime}<t: \mathscr{M}, t^{\prime}, \mathscr{g} \vDash \varphi$;

17. $\mathscr{M}, t, \mathscr{g} \vDash G \varphi$ iff for all $t^{\prime} \in \mathscr{T}$ s. t. $t^{\prime}>t: \mathscr{M}, t^{\prime}, \mathscr{g} \vDash \varphi$.

\section{Definition 29 (Consequence and Truth in a Model)}

For every model $\mathscr{M}$ (based on some frame $\mathscr{F}$ ), wff $\varphi$ and set of wffs $\Gamma$ :

1. $\varphi$ is a consequence of $\Gamma$ in $\mathscr{M}, \Gamma \vDash \mathscr{M} \varphi$, if and only if

$\forall g \in A s \mathscr{F} \forall t \in \mathscr{T}(\forall \gamma \in \Gamma(\mathscr{M}, t, \mathscr{g} \vDash \gamma) \Rightarrow \mathscr{M}, t, \mathfrak{g} \vDash \varphi)$.

2. $\varphi$ is true in $\mathscr{M}, \vDash_{\mathscr{M}} \varphi$, if and only if $\emptyset \vDash_{\mathscr{M}} \varphi$.

\section{Definition 30 ( $\mathrm{L}_{\mathrm{E}}$ Consequence and Truth)}

For every wff $\varphi$ and set of wffs $\Gamma$ :

1. $\varphi$ is an $\mathrm{L}_{\mathrm{E}}$-consequence of $\Gamma, \Gamma \vDash \varphi$, if and only if, for every model $\mathscr{M}: \Gamma \vDash \mathscr{M} \varphi$;

2. $\varphi$ is an $\mathrm{L}_{\mathrm{E}}$-truth, $\vDash \varphi$, if and only if $\emptyset \vDash \varphi$.

We will call the theory consisting of all $\mathrm{L}_{\mathrm{E}}$ truths the E-theory. 
This concludes the presentation of the model theory for $\mathrm{L}_{\mathrm{E}}$. In the next section we discuss a couple illustrative examples concerning the predictions made by the E-theory.

\section{Illustrative Examples}

The Ham Sandwich And now, "we can give a satisfactory account of the ham sandwich" [10, p. 67], which is a rigid embodiment $s=s_{1}, h, s_{2} / B$ consisting of two slices of bread, $s_{1}$ and $s_{2}$, and a slice of ham, $h$, and is structured by the 'betweenness' relation, $B$.

Let $\mathscr{M}=\left\langle\mathscr{T}, \mathscr{P}, d_{0}, \kappa, \leq, v\right\rangle$ be a model such that:

1. $\mathscr{T}=\{1,2,3\}$;

2. $d_{0,1}=d_{0,2}=d_{0,3}=\left\{\jmath_{1}, h, s_{2}\right\}$;

3. $1 \leq 2 \leq 3$;
4. $v\left(s_{1}\right)=s_{1}, v\left(s_{2}\right)=s_{2}$ and $v(h)=h$;

5. $\quad v(B)=\mathscr{B}$ s.t.: $\mathscr{B}(1)=\emptyset$; $\mathscr{B}(2)=\mathscr{B}(3)=\left\{\left\langle s_{1}, h, s_{2}\right\rangle\right\}$.

Then, for every $q \in A s_{\mathscr{F}}$ :

$$
\begin{aligned}
v^{g}\left(s_{1}, h, s_{2} / B\right) & =\left\langle\lambda t \cdot v^{q}\left(s_{1}\right), \lambda t \cdot v^{q}(h), \lambda t \cdot v^{q}\left(s_{2}\right), \mathscr{B}\right\rangle \\
& =\left\langle\lambda t . s_{1}, \lambda t . h, \lambda t . s_{2}, \mathscr{B}\right\rangle .
\end{aligned}
$$

Among the consequences of the model theory are the following:

1. (a) $\mathscr{M}, 1, \mathcal{g} \vDash \neg B s_{1} h s_{2}$, for every $\mathcal{g} \in A s_{\mathscr{F}}$;

(b) $\mathscr{M}, 1, \mathcal{L} \vDash \neg \exists x\left(x=\left(s_{1}, h, s_{2} / B\right)\right)$, for every $\mathscr{Z} \in A s_{\mathscr{F}}$ :

(c) $\mathscr{M}, 1, \mathcal{g} \vDash \neg s_{1} \triangleleft\left(s_{1}, h, s_{2} / B\right)$, for every $\mathscr{g} \in A s_{\mathscr{F}}$;

(d) $\mathscr{M}, 1, \mathscr{g} \vDash \neg h \triangleleft\left(s_{1}, h, s_{2} / B\right)$, for every $\mathscr{Z} \in A s \mathscr{F}$;

(e) $\mathscr{M}, 1, \mathcal{g} \vDash \neg s_{2} \triangleleft\left(s_{1}, h, s_{2} / B\right)$, for every $\mathscr{Z} \in A s_{\mathscr{F}}$;

2. (a) $\mathscr{M}, t, \mathscr{q} \vDash B s_{1} h s_{2}$, for every $\mathscr{q} \in A s_{\mathscr{F}}$ and every $t \in\{2,3\}$;

(b) $\mathscr{M}, t, \mathcal{g} \vDash \exists x\left(x=\left(s_{1}, h, s_{2} / B\right)\right)$, for every $\mathscr{g} \in A s_{\mathscr{F}}$ and every $t \in$ $\{2,3\}$

(c) $\mathscr{M}, t, \mathcal{L} \vDash s_{1} \triangleleft\left(s_{1}, h, s_{2} / B\right)$, for every $\mathscr{g} \in A s_{\mathscr{F}}$ and every $t \in\{2,3\}$;

(d) $\mathscr{M}, t, \mathscr{g} \vDash h \triangleleft\left(s_{1}, h, s_{2} / B\right)$, for every $\mathscr{g} \in A s_{\mathscr{F}}$ and every $t \in\{2,3\}$;

(e) $\mathscr{M}, t, \mathcal{g} \vDash s_{2} \triangleleft\left(s_{1}, h, s_{2} / B\right)$, for every $\mathscr{q} \in A s_{\mathscr{F}}$ and every $t \in\{2,3\}$.

The Car Similarly we can now "provide a more satisfactory account of the car" $[10$, p. 68]. We model the change in parts in the car by a variable embodiment / $A$ / consisting at each time of rigid embodiments composed of 'automotive' parts such as a chassis $(a)$, body $(b)$, engine $(e)$, etc. organized in the 'automotive way' $(R)$, and which undergoes a change in its carburettor (from $c$ to $d$ ) and then is disassembled.

Let $\mathscr{M}=\left\langle\mathscr{T}, \mathscr{P}, d_{0}, \kappa, \leq, v\right\rangle$ be a model such that:

1. $\mathscr{T}=\{1,2,3\}$;

2. $d_{0,1}=d_{0,2}=d_{0,3}=\{a, b, c, d, e\}$

3. $1 \leq 2 \leq 3$;

4. $v(a)=a, v(b)=b, v(c)=c, v(d)=d, v(e)=e$; 
5. $v(R)=\mathscr{R}$ such that $\mathscr{R}(1)=\{\langle a, b, c, e\rangle\}, \mathscr{R}(2)=\{\langle a, b, d, e\rangle\}$ and $\mathscr{R}(3)=\emptyset$;

6. $v(A)=\mathscr{A}$ such that $\mathscr{A}(1)=\{\langle\lambda t . a, \lambda t . \mathscr{b}, \lambda t . c, \lambda t . e, \mathscr{R}\rangle\}, \mathscr{A}(2)=$ $\{\langle\lambda t . a, \lambda t . b, \lambda t . d, \lambda t . e, \mathscr{R}\rangle\}$ and $\mathscr{A}(3)=\emptyset$.

Now, let $m: \mathscr{T} \rightarrow \bigcup_{t \in \mathscr{T}}\left(d_{\kappa, t}\right)$ be a function such that:

1. $m(1)=\langle\lambda t . a, \lambda t . \ell, \lambda t . c, \lambda t . e, \mathscr{R}\rangle$;

2. $m(2)=\langle\lambda t . a, \lambda t . b, \lambda t . d, \lambda t . e, \mathscr{R}\rangle$;

3. $m$ is undefined when $t=3$.

Then, for every $q \in A s \mathscr{F}$ :

$$
u^{q}(/ A /)=\left\langle m, v^{q}(A)\right\rangle=\langle m, \mathscr{A}\rangle \text {. }
$$

Among the consequences of the model theory are the following:

1. (a) $M, 1, q \vDash a \prec / A /$, for all $\mathscr{g} \in A s_{\mathscr{F}}$;

(b) $\mathscr{M}, 1, \mathscr{g} \vDash b \prec / A /$, for all $\mathscr{g} \in A s_{\mathscr{F}}$;

(c) $\mathscr{M}, 1, \mathscr{g} \vDash c \prec / A /$, for all $\mathscr{q} \in A s_{\mathscr{F}}$;

(d) $\mathscr{M}, 1, q \vDash \neg d</ A /$, for all $\mathscr{g} \in A s_{\mathscr{F}}$;

2. (a) $\mathscr{M}, 2, \mathscr{g} \vDash a \prec / A /$, for all $\mathscr{g} \in A s_{\mathscr{F}}$;

(b) $\mathscr{M}, 2, \mathscr{q} \vDash b \prec / A /$, for all $\mathscr{g} \in A s_{\mathscr{F}}$;

(c) $\mathscr{M}, 2, q \vDash d \prec / A /$, for all $\mathscr{q} \in A s \mathscr{F}$;

(d) $\mathscr{M}, 2, \mathscr{g} \vDash \neg c</ A /$, for all $\mathscr{g} \in A s_{\mathscr{F}}$.

We now turn to the proof that Fine's theory of embodiments is sound with respect to the semantics offered in Section 3.

\section{Soundness of the Theory of Embodiments}

We begin by formulating the postulates of the theory of embodiments in the language $\mathrm{L}_{\mathrm{E}}$.

\subsection{Fine's Postulates}

Let $\vec{x}_{n}$ and $\vec{y}_{n}$ be, respectively, the sequences of $x_{1}, \ldots, x_{n}$ and $y_{1}, \ldots, y_{n}$ of variables. Fine's postulates are regimented in $\mathrm{L}_{\mathrm{E}}$ as follows:

Rigid Embodiments:

(R1) $\exists x\left(x=\vec{y}_{n} / X^{n}\right) \leftrightarrow X^{n} \vec{y}_{n}$.

(R2) $\exists x\left(x=\vec{y}_{n} / X^{n}\right) \rightarrow \forall p\left(\operatorname{Loc}(p) \vec{y}_{n} / X^{n} \leftrightarrow \bigvee_{1<i<n}\left(\operatorname{Loc}(p) y_{i}\right)\right)$.

(R3) $\exists z\left(z=\vec{x}_{n} / X^{n}\right) \rightarrow\left(\vec{x}_{n} / X^{n}=\vec{y}_{m} / Y^{m} \leftrightarrow\left(\bigwedge_{1 \leq i \leq m}\left(x_{i}=y_{i}\right) \wedge X^{n}=Y^{m}\right)\right)$.

(R4) $\exists z\left(z=\vec{x}_{n} / X^{n}\right) \rightarrow \bigwedge_{1 \leq i \leq n} x_{i} \triangleleft \vec{x}_{n} / X^{n}$.

(R5) $\exists z\left(z=\vec{x}_{n} / X^{n}\right) \rightarrow X^{n} \triangleleft \overrightarrow{\vec{x}}_{n} / X^{n}$.

(R6) $\quad\left(x \triangleleft \vec{y}_{n} / X^{n} \rightarrow \bigvee_{1 \leq i \leq n}\left(x \unlhd y_{i}\right)\right) \wedge\left(Y^{m} \triangleleft \vec{y}_{n} / X^{n} \rightarrow \bigvee_{1 \leq i \leq n}\left(Y^{m} \triangleleft y_{i}\right) \vee Y^{m}=\right.$ $\left.X^{n}\right)$.

Variable Embodiments:

(V1) $\exists x(x=/ X /) \leftrightarrow \exists x(x M / X /)$. 
(V2) $\exists x(x=/ X /) \rightarrow \exists x(x M / X / \wedge \forall p(\operatorname{Loc}(p) / X / \leftrightarrow \operatorname{Loc}(p) x))$.

(V3) $\exists x(x=/ X /) \rightarrow(/ X /=/ Y / \leftrightarrow X=Y)$.

(V4) $\quad x M / X / \rightarrow x \prec / X /$.

(V5a) $\quad(\exists y(\exists u(x=u) \wedge x \triangleleft y \wedge y \prec z) \rightarrow x \prec z) \wedge\left(\exists y\left(\exists U^{n}\left(X^{n}=U^{n}\right) \wedge X^{n} \triangleleft\right.\right.$ $\left.y \wedge y \prec z) \rightarrow X^{n} \prec z\right)$.

(V5b) $\quad\left(\exists y(x \prec y \wedge(\exists u(z=u) \wedge y \triangleleft z) \rightarrow x \prec z) \wedge \exists y\left(X^{n} \prec y \wedge \exists u(z=\right.\right.$ u) $\left.\wedge y \triangleleft z) \rightarrow X^{n} \prec z\right)$.

(V6) $\quad(x \prec y \rightarrow x \rightsquigarrow y) \wedge\left(X^{n} \prec y \rightarrow X^{n} \rightsquigarrow y\right)$.

Before proceeding it is relevant to make a couple of observations on the present formulation of Fine's postulates. The first observation is that the formulation of principles (R3), (R4), (R5) and (V3) differs slightly from the one offered in Section 1. The present formulation qualifies the principles presented in Section 1 by making them conditional on the existence of the requisite rigid or variable embodiments. To see the need for such qualification, consider, for instance, principle (R3). In general, the parts of a past or future rigid embodiment may all exist, and so may all be self-identical, even though the rigid embodiment is not self-identical, as it does not currently exist (recall that in our models standing in a relation at a time implies existence at that time). The antecedent of (R3) rules out this scenario by requiring the existence of the rigid embodiment. Similar remarks apply to principles (R4), (R5) and (V3). Unqualified, the principles presented in Section 1 may fail to be true in hylomorphic models, owing to the fact that the requisite embodiments do not exist.

The second observation concerns principles (R6), (V5a), (V5b) and (V6). These are formulated as conjunctions in order to reflect the fact that not only individuals but also relations may be parts. In each case taking just the first conjunct would not do, since the variables of $\mathrm{L}_{\mathrm{E}}$ do not range over a common domain of individuals and relations.

\subsection{Preliminary Lemmas}

We now state a number of lemmas that will be appealed to in the proof that Fine's postulates are all $\mathrm{L}_{\mathrm{E}}$-truths.

Lemma 2 For every time $t \in \mathscr{T}$, parthood at $t$ is a partial order. That is, parthood at $t$ is reflexive, antisymmetric and transitive in $d_{\kappa, t} \cup \mathscr{D}_{\kappa}^{+}$.

The proof of Lemma 2 depends on Lemmas 3 and 4:

Lemma 3 For every $e=\left\langle m_{1}, \ldots, m_{n}, \mathscr{X}\right\rangle \in \mathscr{B}_{\kappa} \cap\left(\mathscr{R}_{\kappa}^{n} \cup \mathscr{V}_{\kappa}\right)$ and time $t \in \mathscr{T}$, every immediate part of $e$ at $t$ belongs to the transitive closure of e under membership.

Proof of Lemma 3 Suppose $t$ is an arbitrary member of $\mathscr{T}, e=\left\langle m_{1}, \ldots, m_{n}, \mathscr{X}\right\rangle$ is an arbitrary element of $\mathscr{B}_{\kappa} \cap\left(\mathscr{R}_{\kappa}^{n} \cup \mathscr{V}_{\kappa}\right)$ and $x$ is an arbitrary immediate part of $e$ at $t$. Either (a) $x=\mathscr{X}$, or (b) $\exists j \leq n\left(x=m_{j}(t)\right)$, by Definition 9. Clearly, if (a) is true, then $x \in T C(e)$ (where $T C(e)$ is the transitive closure of $e$ under membership). 
Furthermore, if (b) is true, then $\left\langle t, m_{j}(t)\right\rangle \in m_{j}$. So, $m_{j}(t) \in T C\left(m_{j}\right)$. But $m_{j} \in T C(e)$. Therefore, $m_{j}(t) \in T C(e)$. Hence, $x \in T C(e)$.

Lemma 4 For every $n \in \mathbb{Z}^{+}$and every immediate parthood at $t$ sequence $\vec{x}_{n}=$ $\left\langle x_{1}, \ldots, x_{n}\right\rangle$ :

$\forall j \in \mathbb{Z}^{+}: j<n\left(x_{j}\right.$ belongs to the transitive closure of $\left.x_{n}\right)$.

Proof of Lemma 4 The proof is by induction on the positive integers:

(Base Case): when $n=1$ there is no positive integer smaller than $n$. Therefore, the base case holds vacuously.

(Induction Case): By the induction hypothesis, $\forall j \in \mathbb{Z}^{+}: j<n\left(x_{j} \in T C\left(x_{n}\right)\right)$. But, $x_{n} \in T C\left(x_{n+1}\right)$, by Lemma 3. Therefore, $\forall j \in \mathbb{Z}^{+}: j \leq n\left(m_{j} \in\right.$ $\left.T C\left(x_{n+1}\right)\right)$.

We now prove that parthood is a partial order:

\section{Proof of Lemma 2}

(Reflexivity): Follows straightforwardly from Definition 12 , since every $x \in d_{\kappa, t} \cup$ $\mathscr{D}_{\mathcal{K}}^{+}$is such that $x=x$, and so $x \leq_{\mathscr{F}}^{t} x$.

(Antisymmetry): Suppose $x \leq_{\mathscr{F}}^{t} y$ and $y \leq_{\mathscr{F}}^{t} x$. Suppose, for reductio, that $x \neq y$. Then, by Definition 12:

$\exists n, m \in \mathbb{Z}^{+} \exists \vec{x}_{n} \exists \vec{y}_{m}\left(\ll_{\mathscr{F}}^{t}\left(\vec{x}_{n}\right) \& \ll_{\mathscr{F}}^{t}\left(\vec{y}_{m}\right) \& x=x_{1}=y_{m} \& y=y_{1}=\right.$ $\left.x_{n} \& x, y \in d_{\kappa, t}\right)$

By Lemma 4, $x \in T C(y)$ and $y \in T C(x)$. But this is impossible, as it would violate the axiom of foundation. Therefore, $x=y$. Hence, parthood at $t$ is antisymmetric.

(Transitivity): Suppose $x \leq_{\mathscr{F}}^{t} y$ and $y \leq_{\mathscr{F}}^{t} \varkappa$. Then,

$\exists n, m \in \mathbb{Z}^{+} \exists \vec{x}_{n} \exists \vec{y}_{m}\left(\ll_{\mathscr{F}}^{t}\left(\vec{x}_{n}\right) \& \ll_{\mathscr{F}}^{t}\left(\vec{y}_{m}\right) \& x=x_{1} \& y=x_{n}=y_{1} \& \%=\right.$ $\left.y_{m}\right)$, by Definitions 11,12 .

Now, consider the $n+m-1$-sequence $\vec{\varkappa}_{n+m-1}=\left\langle\varkappa_{1}, \ldots, \varkappa_{n}, \ldots, \varkappa_{m+n-1}\right\rangle$ such that:

- $\forall i: 1 \leq i \leq n\left(\varkappa_{i}=x_{i}\right) ;$ and

- $\forall l: 1 \leq l \leq m\left(\varkappa_{l+n-1}=y_{l}\right)$.

Clearly, $\ll_{\mathscr{F}}^{t}\left(\vec{\varkappa}_{n+m-1}\right), \varkappa_{1}=x$ and $\varkappa_{m+n-1}=\varkappa$, by Definitions 9 and 10 . Therefore, $x \leq_{\mathscr{F}}^{t} \approx$, by Definitions 11 and 12 . Thus, parthood at $t$ is transitive. Therefore, parthood at $t$ is a partial order.

\subsection{Soundness}

We now prove that Fine's postulates are sound with respect to the model-theoretic semantics offered in Section 3 by showing that each postulate is an $L_{E}$-truth.

Theorem 1 (Soundness) Fine's theory of embodiments is sound with respect to the semantics characterised in Section 3. 


\section{Proof of Theorem 1}

In what follows we prove that all postulates of Fine's theory are $\mathrm{L}_{\mathrm{E}}$-truths. We do so by showing that every one of Fine's postulates is true at an arbitrary model $\mathscr{M}$ (based on some frame $\mathscr{F}$ ), time $t \in \mathscr{T}$ and variable-assignment $\mathcal{g} \in A s \mathscr{F}$.

Proof of $\mathscr{M}, t, q \vDash(R I)$

$(\Rightarrow)$ : Suppose $\mathscr{M}, t, \mathcal{g} \vDash \exists x\left(x=\vec{y}_{n} / X^{n}\right)$. Then, $v^{q}\left(\vec{y}_{n} / X^{n}\right) \in d_{\kappa, t}$, by Definition 28. So, $v^{q}\left(\vec{y}_{n} / X^{n}\right)=\left\langle\lambda t \in \mathscr{T} \cdot v^{q}\left(y_{1}\right), \ldots, \lambda t \in \mathscr{T} \cdot v^{q}\left(y_{n}\right), v^{q}\left(X^{n}\right)\right\rangle \in$ $d_{\kappa, t} \cap \mathscr{R}_{\kappa}^{n}$, by Definition 27. Hence, $\left\langle v^{q}\left(y_{1}\right), \ldots, v^{q}\left(y_{n}\right)\right\rangle \in v^{q}\left(X^{n}\right)(t)$, by Definition 7. So, $\mathscr{M}, t, q \vDash X^{n} \vec{y}_{n}$, by Definition 28 .

$(\Leftarrow)$ : Suppose $\mathscr{M}, t, q \vDash X^{n} \vec{y}_{n}$. Then, $\left\langle v^{q}\left(y_{1}\right), \ldots, v^{q}\left(y_{n}\right)\right\rangle \in v^{q}\left(X^{n}\right)(t)$, by Definition 28. So, $\left\langle\lambda t \in \mathscr{T} \cdot v^{q}\left(y_{1}\right), \ldots, \lambda t \in \mathscr{T} \cdot v^{q}\left(y_{n}\right), v^{q}\left(X^{n}\right)\right\rangle \in d_{\kappa, t} \cap R_{\kappa}^{n}$ by Definition 7. But $\mho^{\mathscr{q}}\left(\vec{y}_{n} / X^{n}\right)=\left\langle\lambda t \in \mathscr{T} \cdot v^{q}\left(y_{1}\right), \ldots, \lambda t \in \mathscr{T} \cdot v^{q}\left(y_{n}\right), v^{q}\left(X^{n}\right)\right\rangle$, by Definition 27. Therefore, $v^{q}\left(\vec{y}_{n} / X^{n}\right) \in d_{\kappa, t}$. Hence, $\mathscr{M}, t, \mathcal{g} \vDash \exists x(x=$ $\left.\vec{y}_{n} / X^{n}\right)$, by Definition 28 .

Therefore, $\mathscr{M}, t, \mathfrak{g} \vDash \exists x\left(x=\vec{y}_{n} / X^{n}\right) \leftrightarrow X^{n} \vec{y}_{n}$, by Definition 28 .

Proof of $\mathscr{M}, t, q \vDash(R 2)$

Suppose $\mathscr{M}, t, \mathcal{Q} \vDash \exists x\left(x=\vec{y}_{n} / X^{n}\right)$. Then, $v^{q}\left(\vec{y}_{n} / X^{n}\right)=\langle\lambda t \in$ $\left.T . u^{q}\left(y_{1}\right), \ldots, \lambda t \in T . v^{q}\left(y_{n}\right), u^{q}\left(X^{n}\right)\right\rangle \in d_{\kappa, t}$, by Definitions 27 and 28. Let $q^{\prime}$ be any variable-assignment differing from $q$ at most in the value assigned to the spatial variable $p$. We show that $\mathscr{M}, t, g^{\prime} \vDash \operatorname{Loc}(p) \vec{y}_{n} / X^{n}$ iff $\mathscr{M}, t, g^{\prime} \vDash$ $\bigvee_{1 \leq i \leq n} \operatorname{Loc}(p) y_{i}$.

$\Leftrightarrow)$ Suppose that $\mathscr{M}, t, g^{\prime} \vDash \operatorname{Loc}(p) \vec{y}_{n} / X^{n}$. Then, $v^{\prime}\left(\vec{y}_{n} / X^{n}\right)=\langle\lambda t \in$ $\left.T \cdot v^{q^{\prime}}\left(y_{1}\right), \ldots, \lambda t \in T \cdot v^{q^{\prime}}\left(y_{n}\right), v^{q^{\prime}}\left(X^{n}\right)\right\rangle \in d_{\kappa, t, g^{\prime}(p)}$, by Definitions 27 and 28. So, $\exists 1 \leq i \leq n$ s. t. $\lambda t \in \mathscr{T} \cdot v^{q^{\prime}}\left(y_{i}\right)(t)=v^{q^{\prime}}\left(y_{i}\right) \in d_{\kappa, t, g^{\prime}(p)}$, by Definition 8 , since $v^{q}\left(\vec{y}_{n} / X^{n}\right) \in d_{\kappa, t}$, by assumption, and $v^{q}\left(\vec{y}_{n} / X^{n}\right)=v^{q^{\prime}}\left(\vec{y}_{n} / X^{n}\right)-$ as no spatial variable occurs in $\left\ulcorner\vec{y}_{n} / X^{n}\right\urcorner$. So, $\exists 1 \leq i \leq n$ s. t. $\mathscr{M}, t, \mathscr{g}^{\prime} \vDash \operatorname{Loc}(p) y_{i}$, by Definition 28. Hence, $\mathscr{M}, t, \mathcal{g}^{\prime} \vDash \bigvee_{1 \leq i \leq n} \operatorname{Loc}(p) y_{i}$, by Definition 28 .

$(\Leftarrow)$ Suppose $\mathscr{M}, t, \mathscr{g}^{\prime} \vDash \bigvee_{1 \leq i \leq n} \operatorname{Loc}(p) y_{i}$. Then, $\exists 1 \leq i \leq n$ s. t. $\mathscr{M}, t, g^{\prime} \vDash$ $\operatorname{Loc}(p) y_{i}$, by Definition 28. So, $\exists 1 \leq i \leq n$ s. t. $v^{q^{\prime}}\left(y_{i}\right)=\lambda t \in \mathscr{T} \cdot v^{q^{\prime}}\left(y_{i}\right)(t) \in$

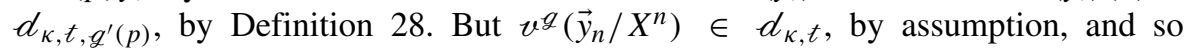
$v^{q^{\prime}}\left(\vec{y}_{n} / X^{n}\right)=\left\langle\lambda t \in T \cdot v^{q^{\prime}}\left(y_{1}\right), \ldots, \lambda t \in T \cdot v^{q^{\prime}}\left(y_{n}\right), v^{q^{\prime}}\left(X^{n}\right)\right\rangle \in d_{\kappa, t, g^{\prime}(p)}$, by Definition 8. Therefore, $\mathscr{M}, t, g^{\prime} \vDash \operatorname{Loc}(p) \vec{y}_{n} / X^{n}$, by Definition 28 .

So, $\mathscr{M}, t, g \vDash \forall p\left(\operatorname{Loc}(p) \vec{y}_{n} / X^{n} \leftrightarrow \bigvee_{1 \leq i \leq n} \operatorname{Loc}(p) y_{i}\right)$, by Definition 28 .

Therefore, $\mathscr{M}, t, \mathcal{F} \exists x\left(x=\vec{y}_{n} / X^{n}\right) \rightarrow \forall p\left(\operatorname{Loc}(p) \vec{y}_{n} / X^{n} \leftrightarrow\right.$ $\left.\bigvee_{1 \leq i \leq n} \operatorname{Loc}(p) y_{i}\right)$, by Definition 28 .

Proof of $\mathscr{M}, t, q \vDash(R 3)$

Suppose $\mathscr{M}, t, \mathcal{g} \vDash \exists z\left(z=\vec{x}_{n} / X^{n}\right)$. Then $v^{q}\left(\vec{x}_{n} / X^{n}\right)=\langle\lambda t \in$ $\left.\mathscr{T} . v^{q}\left(x_{1}\right), \ldots, \lambda t \in \mathscr{T} . v^{q}\left(x_{n}\right), v^{q}\left(X^{n}\right)\right\rangle \in d_{\kappa, t} \cap \mathscr{R}_{\kappa}^{n}$, by Definitions 27 and 28. Furthermore, for all $1 \leq i \leq n, \lambda t \in \mathscr{T}$. $v^{q}\left(x_{i}\right) \in d_{\kappa, t}$, by Definitions 2 and 7 . We show that $\mathscr{M}, t, g \vDash \vec{x}_{n} / X^{n}=\vec{y}_{m} / Y^{m}$ iff $\mathscr{M}, t, q \vDash \bigwedge_{1<i<m} x_{i}=y_{i} \wedge X^{n}=Y^{m}$. $(\Rightarrow)$ Suppose $\mathscr{M}, t, q \vDash \vec{x}_{n} / X^{n}=\vec{y}_{m} / Y^{m}$. Then, $m=n, \lambda t \in \mathscr{T}$. ${ }^{q}\left(x_{i}\right)=\lambda t \in$ $\mathscr{T} \cdot v^{q}\left(y_{i}\right) \in d_{\kappa, t}$, for all $1 \leq i \leq n$ and $\vartheta^{q}\left(X^{n}\right)=v^{q}\left(Y^{n}\right)$, by the assumption and 
Definition 27. So, $\mathscr{M}, t, q \vDash x_{i}=y_{i}$ for all $1 \leq i \leq m(=n), \mathscr{M}, t, q \vDash X^{n}=Y^{m}$, by Definition 28. So, $\mathscr{M}, t, q \vDash \bigwedge_{1 \leq i \leq m} x_{i}=y_{i} \wedge X^{n}=Y^{m}$, by Definition 28 .

$(\Leftarrow)$ Suppose $\mathscr{M}, t, q \vDash \bigwedge_{1 \leq i \leq m} x_{i}=y_{i} \wedge X^{n}=Y^{m}$. Then, $m=n, \lambda t \in$ $\mathscr{T} \cdot u^{q}\left(x_{i}\right)=\lambda t \in \mathscr{T} \cdot v^{q}\left(y_{i}\right) \in d_{\kappa, t}$, for all $1 \leq i \leq n$ and $v^{q}\left(X^{n}\right)=v^{q}\left(Y^{n}\right)$, Definition 28. So, $\left\langle\lambda t \in \mathscr{T} \cdot v^{q}\left(x_{1}\right), \ldots, \lambda t \in \mathscr{T} \cdot v^{q}\left(x_{n}\right), v^{q}\left(X^{n}\right)\right\rangle=\langle\lambda t \in$ $\left.\mathscr{T} \cdot v^{q}\left(y_{1}\right), \ldots, \lambda t \in \mathscr{T} \cdot v^{q}\left(y_{n}\right), v^{q}\left(Y^{n}\right)\right\rangle$. But, by assumption, $v^{q}\left(\vec{x}_{n} / X^{n}\right)=$ $\left\langle\lambda t \in \mathscr{T} \cdot v^{q}\left(x_{1}\right), \ldots, \lambda t \in \mathscr{T} \cdot v^{q}\left(x_{n}\right), v^{q}\left(X^{n}\right)\right\rangle \in d_{\kappa, t} \cap \mathscr{R}_{\kappa}^{n}$. So, $v^{q}\left(\vec{x}_{n} / X^{n}\right)=$ $v^{q}\left(\vec{y}_{m} / Y^{m}\right) \in d_{\kappa, t}$, by Definition 27. So, $\mathscr{M}, t, \mathcal{g} \vDash \vec{x}_{n} / X^{n}=\vec{y}_{m} / Y^{m}$, by Definition 28.

Therefore, $\mathscr{M}, t, g \vDash \vec{x}_{n} / X^{n}=\vec{y}_{m} / Y^{m} \leftrightarrow \bigwedge_{1<i<m} x_{i}=y_{i} \wedge X^{n}=Y^{m}$, by Definition 28.

Hence, $\mathscr{M}, t, q \vDash \exists z\left(z=\vec{x}_{n} / X^{n}\right) \rightarrow \vec{x}_{n} / X^{n}=\vec{y}_{m} / Y^{m} \leftrightarrow \bigwedge_{1 \leq i \leq m} x_{i}=y_{i} \wedge$ $X^{n}=Y^{m}$, by Definition 28 .

Proof of $\mathscr{M}, t, q \vDash(R 4)$ and $(R 5)$

Suppose $\mathscr{M}, t, \mathcal{L} \vDash \exists z\left(z=\vec{x}_{n} / X^{n}\right)$. Then $v^{q}\left(\vec{x}_{n} / X^{n}\right)=\langle\lambda t \in$ $\left.\mathscr{T} . v^{q}\left(x_{1}\right), \ldots, \lambda t \in \mathscr{T} . v^{q}\left(x_{n}\right), v^{q}\left(X^{n}\right)\right\rangle \in d_{\kappa, t} \cap \mathscr{R}_{\kappa}^{n}$, by Definitions 27 and 28. Furthermore, $v^{q}\left(x_{i}\right)=\lambda t \in \mathscr{T}$. $v^{q}\left(x_{i}\right)(t)$, for all $t \in T$ and each $1 \leq i \leq n$. Since $v^{q}\left(\vec{x}_{n} / X^{n}\right) \in d_{\kappa, t} \cap \mathscr{R}_{\kappa}^{n}$, it follows by Definitions 2 and 7 that $v^{\bar{q}}\left(x_{i}\right) \in d_{\kappa, t}$, for all $t \in T$ and each $1 \leq i \leq n$. Furthermore, $v^{q}\left(X^{n}\right) \in \mathscr{D}_{\kappa}^{n}$. So, by Definition 9, for each $1 \leq i \leq n, v^{q}\left(x_{i}\right)$ and $v^{q}\left(X^{n}\right)$ are immediate parts at $t$ of $\left\langle\lambda t \in \mathscr{T} \cdot v^{q}\left(x_{1}\right), \ldots, \lambda t \in \mathscr{T} \cdot v^{q}\left(x_{n}\right), v^{q}\left(X^{n}\right)\right\rangle \in \mathscr{R}_{\kappa}^{n}$. So:

For each $1 \leq i \leq n \mathscr{M}, v^{q}\left(x_{i}\right) \triangleleft_{\mathscr{F}}^{t} v^{q}\left(\vec{x}_{n} / X^{n}\right)$, by Definition 13. So, for each $1 \leq i \leq n, \bar{M}, t, q \vDash \bigwedge_{1 \leq i \leq n} x_{i} \triangleleft \vec{x}_{n} / X^{n}$, by Definition 28. Therefore, $\mathscr{M}, t, q \vDash$ $\exists z\left(z=\vec{x}_{n} / X^{n}\right) \rightarrow \bigwedge_{1 \leq i \leq n} x_{i} \triangleleft \vec{x}_{n} / X^{n}$, by Definition 28. This establishes that $\mathscr{M}, t, q \vDash(\mathrm{R} 4)$.

$\mathscr{M}, t, g \vDash X^{n} \triangleleft \vec{x}_{n} / X^{n}$, by Definition 28. Therefore, $\mathscr{M}, t, g \vDash \exists z\left(z=\vec{x}_{n} / X^{n}\right) \rightarrow$ $X^{n} \triangleleft \vec{x}_{n} / X^{n}$, by Definition 28. This establishes that $\mathscr{M}, t, g \vDash(\mathrm{R} 5)$.

Proof of $\mathscr{M}, t, q \vDash(R 6)$

Without loss of generality, we show only that $\mathscr{M}, t, \mathcal{g} \vDash x \triangleleft \vec{y}_{n} / X^{n} \rightarrow$ $\bigvee_{1 \leq i \leq n}\left(x \unlhd y_{i}\right)$. Suppose $\mathscr{M}, t, q \vDash x \triangleleft \vec{y}_{n} / X^{n}$. Then, $v^{q}(x) \triangleleft_{\mathscr{F}}^{t} v^{q}\left(\vec{y}_{n} / X^{n}\right)$, by Definition 28. By Definition 27, $v^{q}\left(\vec{y}_{n} / X^{n}\right)=\left\langle\lambda t \in \mathscr{T} \cdot v^{q}\left(y_{1}\right), \ldots, \lambda t \in\right.$ $\left.\mathscr{T} \cdot v^{q}\left(y_{n}\right), v^{q}\left(X^{n}\right)\right\rangle \in d_{\kappa, t} \cap \mathscr{R}_{\kappa}^{n}$. So, by Definition 9 , the only immediate parts at $t$ of $v^{q}\left(\vec{y}_{n} / X^{n}\right)$ are $v^{q}\left(y_{i}\right)$, for each $i: 1 \leq i \leq n$, and $v^{q}\left(X^{n}\right)$. Since $v^{q}(x) \triangleleft_{\mathscr{F}}^{t} v^{q}\left(\vec{y}_{n} / X^{n}\right)$, there is an immediate parthood at $t$ sequence $\vec{x}_{m+1}$ linking $v^{q}(x)$ to $v^{q}\left(\vec{y}_{n} / X^{n}\right)$ and such that, for each $1<i<n, x_{i} \in \mathscr{D}_{\kappa}^{+}$or $x_{i+1} \in \mathscr{R}_{\kappa}$, by Definition 11. So, clearly, there is some $i: 1 \leq i \leq n$ such that $v^{q}\left(y_{i}\right)$ is the $m$ th-element of $\vec{x}_{m+1}$, and so $v^{q}\left(y_{i}\right) \in d_{\kappa, t} \cap \mathscr{R}_{\kappa}$. But then, there is some $i: 1 \leq i \leq n$ such that either $\mho^{q}\left(y_{i}\right)=\mho^{q}(x)$, or else $\vec{x}_{m}$ is an immediate parthood at $t$ sequence linking $v^{q}(x)$ to $v^{q}\left(y_{i}\right)$, and such that, for each $1<i<n, x_{i} \in \mathscr{D}_{\kappa}^{+}$ or $x_{i+1} \in \mathscr{R}_{\kappa}$. So, there is an $i: 1 \leq i \leq n$ such that either $\mathscr{M}, t, \mathcal{g} \vDash x=y_{i}$ or else $v^{q}(x) \triangleleft_{\mathscr{F}}^{t} v^{q}\left(y_{i}\right)$, by Definitions 13 and 28. So, $\mathscr{M}, t, g \vDash \bigvee_{1<i<n}\left(x \unlhd y_{i}\right)$, by Definition 28. Therefore, $\mathscr{M}, t, q \vDash x \triangleleft \vec{y}_{n} / X^{n} \rightarrow \bigvee_{1 \leq i \leq n}\left(x \unlhd y_{i}\right)$. 
Proof of $\mathscr{M}, t, q \vDash(V 1)$

$(\Rightarrow)$ : Suppose $\mathscr{M}, t, q \vDash \exists x(x=/ X /)$. Then, $v^{q}(/ X /) \in d_{\kappa, t}$, by Definition 28 . So, $\exists m\left(v^{q}(/ X /)=\left\langle m, v^{q}(X)\right\rangle \in \mathscr{V}_{\kappa} \cap d_{\kappa, t}\right)$, by Definition 27. Therefore, $\exists x \in$ $d_{\kappa, t}\left(m(t)=x \& x M_{\mathscr{F}}^{t} v^{q}(/ X /)\right)$, by Definitions 2,7 and 15 . So, $\mathscr{M}, t, q \vDash$ $\exists x(x M / X /))$, by Definition 28 .

$(\Leftarrow)$ : Suppose $\mathscr{M}, t, q \vDash \exists x(x M / X /))$. Then, $\exists x \in d_{\kappa, t}\left(x M_{\mathscr{F}}^{\mathscr{T}} \mho^{q}(/ X /)\right)$. So, $u^{q}(/ X /) \in d_{\kappa, t}$, by Definition 15. So, $\mathscr{M}, t, q \vDash \exists x(x=/ X /)$, by Definition 28 . Therefore, $\mathscr{M}, t, \mathscr{g} \vDash \exists x(x M / X /)) \leftrightarrow \exists x(x=/ X /)$, by Definition 28 .

Proof of $\mathscr{M}, t, q \vDash(V 2)$

Suppose $\mathscr{M}, t, \mathcal{g} \vDash \exists x(x=/ X /)$. Then, as shown in the proof of $\mathscr{M}, t, g \vDash(\mathrm{V} 1)$, $\exists x \in d_{\kappa, t}\left(m(t)=x \& x M_{\mathscr{F}}^{t} v^{q}(/ X /) \& v^{q}(/ X /) \in \mathscr{V}_{\kappa} \cap d_{\kappa, t}\right)$. Now, let $g^{\prime}$ be any variable-assignment differing from the variable-assignment $g[x / m(t)]$ at most in the value assigned to the spatial variable $p$. We show that $\mathscr{M}, t, \mathscr{q}^{\prime} \vDash \operatorname{Loc}(p) / X /$ iff $\mathscr{M}, t, q^{\prime} \vDash \operatorname{Loc}(p) x$.

$(\Rightarrow)$ : Suppose $\mathscr{M}, t, \mathscr{q}^{\prime} \vDash \operatorname{Loc}(p) / X /$. Then, $v^{q^{\prime}}(/ X /) \in d_{\kappa, t, q^{\prime}(p)}$, by Definition 28. So, $m(t) \in d_{\kappa, t, g^{\prime}(p)}$, by Definition 8 , since $v^{q^{\prime}}(/ X /) \in \mathscr{V}_{\kappa}$. So, $\mathscr{M}, t, g^{\prime} \vDash \operatorname{Loc}(p) x$, by Definitions 27 and 28, since $q[x / m(t)](x)=m(t)$.

$(\Leftarrow)$ : Suppose $\mathscr{M}, t, \mathscr{q}^{\prime} \vDash \operatorname{Loc}(p) x$. Then $m(t) \in d_{\kappa, t, g^{\prime}(p)}$, by Definitions 27 and 28. But $v^{q}(/ X /)=\left\langle m, v^{q^{\prime}}(X)\right\rangle \in \mathscr{V}_{\kappa} \cap d_{\kappa, t}$, by the main assumption. So, by Definition $8, v^{q}(/ X /) \in d_{\kappa, t, g^{\prime}(p)}$. Hence, $\mathscr{M}, t, g^{\prime} \vDash \operatorname{Loc}(p) / X /$, by Definition 28.

So, $\mathscr{M}, t, \mathscr{q}^{\prime} \vDash \operatorname{Loc}(p) / X / \leftrightarrow \operatorname{Loc}(p) x$, by Definition 28. Hence, $\mathscr{M}, t, g[x / m(t)] \vDash \forall p(\operatorname{Loc}(p) / X / \leftrightarrow \operatorname{Loc}(p) x)$, by Definition 28. So, $\mathscr{M}, t, q[x / m(t)] \vDash x M / X / \wedge \forall p(\operatorname{Loc}(p) / X / \leftrightarrow \operatorname{Loc}(p) x)$, by Definition 28 and the main assumption. Therefore, $\mathscr{M}, t, q \vDash \exists x(x M / X / \wedge \forall p(\operatorname{Loc}(p) / X / \leftrightarrow$ $\operatorname{Loc}(p) x))$, by Definition 28. Hence, $\mathscr{M}, t, q \vDash \exists x(x=/ X /) \rightarrow \exists x(x M / X / \wedge$ $\forall p(\operatorname{Loc}(p) / X / \leftrightarrow \operatorname{Loc}(p) x))$, by Definition 28 .

Proof of $\mathscr{M}, t, q \vDash(V 3)$

Suppose $\mathscr{M}, t, q \vDash \exists x(x=/ X /)$. Then, $\boldsymbol{v}^{q}(/ X /) \in d_{\kappa, t}$ by Definition 28. Furthermore, there is an $m$ such that $v^{q}(/ X /)=\left\langle m, v^{q}(X)\right\rangle \in \mathscr{V}_{\kappa}$, by Definition 27. We now show that $\mathscr{M}, t, q \vDash / X /=/ Y /$ iff $\mathscr{M}, t, q \vDash X=Y$, by Definition 28 . $(\Rightarrow)$ : Suppose $\mathscr{M}, t, q \vDash / X /=/ Y /$. Then, $v^{q}(/ X /)=v^{q}(/ Y /) \in d_{\kappa, t}$, by Definition 28. So, $\left\langle m, v^{q}(Y)\right\rangle=v^{q}(/ Y /)=v^{q}(/ X /)=\left\langle m, v^{q}(X)\right\rangle \in d_{\kappa, t} \cap$ $\mathscr{V}_{\kappa}$, by Definitions 27 and 28. So, $v^{\mathscr{q}}(X)=v^{\mathscr{q}}(Y) \in \mathscr{D}_{\kappa}^{1}$, by Definition 27. Thus, $\mathscr{M}, t, q \vDash X=Y$, by Definition 28 .

$(\Leftarrow)$ : Suppose that $\mathscr{M}, t, q \vDash X=Y$. Then $v^{q}(X)=v^{q}(Y) \in \mathscr{D}_{\kappa}^{1}$, by Definitions 28 and 27. By assumption, there is an $m$ such that $v^{q}(/ X /)=\left\langle m, v^{q}(X)\right\rangle \in$ $d_{\kappa, t} \cap \mathscr{V}_{\kappa}$. So, $v^{q}(/ X /)=\left\langle m, v^{q}(Y)\right\rangle \in d_{\kappa} \cap \mathscr{V}_{\kappa}$. But then, $v^{q}(/ Y /)=$ $\left\langle m, v^{q}(Y)\right\rangle \in d_{\kappa} \cap \mathscr{V}_{\kappa}$, by Definition 27. So, $v^{q}(/ X /)=v^{q}(/ Y /) \in d_{\kappa, t}$. Therefore, $\mathscr{M}, t, q \vDash / X /=/ Y /$, by Definition 28 .

Therefore, $\mathscr{M}, t, g \vDash / X /=/ Y / \leftrightarrow X=Y$, by Definition 28. Hence, $\mathscr{M}, t, g \vDash$ $\exists x(x=/ X /) \rightarrow(/ X /=/ Y / \leftrightarrow X=Y)$, again by Definition 28 . 
Proof of $\mathscr{M}, t, q \vDash(V 4)$

Suppose $\mathscr{M}, t, \mathscr{g} \vDash x M / X /$. Then, $\mho^{q}(x) M_{\mathscr{F}}^{t} \mho^{\mathscr{q}}(/ X /)$, by Definition 28 , and $u^{q}(x), v^{q}(/ X /) \in d_{\kappa, t}$, by Definition 16. So, $v^{q}(x) \prec_{\mathscr{F}}^{t} u^{q}(/ X /)$, by Definition 15. So, $\mathscr{M}, t, \mathcal{g} \vDash x \prec / X /$, by Definition 28. Therefore, $\mathscr{M}, t, \mathscr{q} \vDash$ $x M / X / \rightarrow x \prec / X /$, by Definition 28 .

Proof of $\mathscr{M}, t, \mathfrak{q} \vDash(V 5)$

Without loss of generality, we consider only the proof of the first conjunct of (V5a). Suppose $\mathscr{M}, t, \mathcal{g} \vDash \exists y(\exists u(x=u) \wedge x \triangleleft y \wedge y \prec z)$. Then, by Definition 28, there is a $y \in d_{\kappa, t}$ s. t.: (i) $v^{q}(x) \in d_{\kappa, t}$; (ii) $v^{q}(x) \triangleleft_{\mathscr{F}}^{t} y$; and (iii) $y \prec_{\mathscr{F}}^{t} v^{q}(z)$. From (iii) it follows that there are $u, w \in d_{\kappa, t}$ such that $u M_{\mathscr{F}}^{t} w, y \leqslant_{\mathscr{F}}^{t} u$ and $w \leqslant_{\mathscr{F}}^{t} u^{q}(z)$, by Definition 16. From (ii) it follows that $v^{q}(x)<_{\mathscr{F}}^{t} y$, by Definitions 11 and 13 . So, from Lemma 2 it follows that $u^{q}(x)<_{\mathscr{F}}^{t} u$. But then there are $u, w \in d_{\kappa, t}$ such that $u M_{\mathscr{F}}^{t} w, v^{q}(x) \leqslant_{\mathscr{F}}^{t} u$ and $w \leqslant_{\mathscr{F}}^{t} v^{q}(z)$. Therefore, $v^{q}(x) \prec_{\mathscr{F}}^{t} v^{q}(z)$, by Definition 16. So, $\mathscr{M}, t, q \vDash x \prec z$, by Definition 28. Hence, $\mathscr{M}, t, q \vDash \exists y(\exists u(x=$ u) $\wedge x \triangleleft y \wedge y \prec z) \rightarrow x \prec z$, by Definition 28 .

\section{Proof of $\mathscr{M}, t, q \vDash(V 6)$}

Without loss of generality, we consider only the proof of the first conjunct of (V6). Suppose $\mathscr{M}, t, \mathscr{q} \vDash x \prec y$. Then, $v^{q}(x) \prec_{\mathscr{F}}^{t} v^{q}(y)$, by Definition 28. So, $\exists u, z \in d_{\kappa, t}\left(u^{q}(x) \leqslant_{\mathscr{F}}^{t} u \& u M_{\mathscr{F}}^{t} z \& \approx \leqslant_{\mathscr{F}}^{t} u^{q}(y)\right)$, by Definition 16. Since $z \leqslant \leqslant_{\mathscr{F}}^{t} u^{q}(y)$, there is a sequence $\vec{z}_{n}$ such that either $\vec{z}_{n}$ is an immediate parthood at $t$ sequence, $\varkappa_{1}=\varkappa$ and $\varkappa_{n}=v^{q}(y)$, or else $\vec{\varkappa}_{n}$ is a one element sequence whose

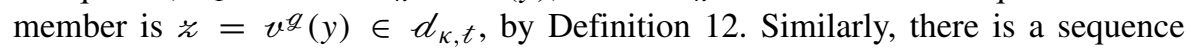
$\vec{u}_{m}$ such that either $\vec{u}_{m}$ is an immediate parthood at $t$ sequence $\vec{u}_{m}$ such that $u_{1}=v^{q}(x)$ and $u_{m}=u$, or else $\vec{u}_{m}$ is a one element sequence whose only member is $v^{q}(x)=u \in d_{\kappa, t}$, by Definition 12. Now, consider the sequence $\vec{x}_{m+n}$ such that $x_{i}=u_{i}$, for all $i: 1 \leq i \leq m$ and $x_{i+m}=\varkappa_{i}$ for all $i: 1 \leq i \leq m$. Clearly, $\vec{x}_{m+n}$, is an immediate parthood sequence at $t$, by Definitions 9,10 and 15 . Furthermore, for every $1 \leq i<m+n, x_{i}=\mathscr{X}$ or $x_{i}=m_{j}(t)$, where $x_{i+1}=\left\langle m_{1}, \ldots, m_{l}, \mathscr{X}\right\rangle$, for some $l \in \mathbb{Z}^{+}, m_{1}, \ldots, m_{l}$ and $\mathscr{X}$. If $x_{i}=\mathscr{X}$ or $x_{i+1}$ is a rigid embodiment, then $x_{i} \triangleleft_{\mathscr{F}}^{t} x_{i+1}$, by Definition 13. Otherwise, $x_{i} M_{\mathscr{F}}^{t} x_{i+1}$, by Definitions 9 and 15. Furthermore, $u=x_{m} M_{\mathscr{F}}^{t} x_{m+1}=\varkappa$. So, $v^{q}(x) \rightsquigarrow_{\mathscr{F}}^{t} v^{q}(y)$, by Definitions 18 and 19. Therefore, $\mathscr{M}, t, q \vDash x \rightsquigarrow y$, by Definition 28. Hence, $\mathscr{M}, t, q \vDash x \prec y \rightarrow x \rightsquigarrow y$, by Definition 28 .

This concludes the proof of Theorem 1. Fine's theory of embodiments is sound with respect to the model-theory offered in Section 3.

\section{Expressibility in the Theory of Embodiments}

In this section we consider a number of results concerning the definability of mereological notions. The two main results of the section are the following: 
timeless parthood and immediate parthood are not definable in terms of parthood; all the mereological primitives of $\mathrm{L}_{\mathrm{E}}$ are definable in terms of immediate parthood.

We begin by showing the exact sense in which timeless parthood and immediate parthood are not definable in terms of parthood. In order to do so we will first define the temporal operators ' $A$ ' (always) and ' $S$ ' (sometimes) in terms of the primitives of $\mathrm{L}_{\mathrm{E}}$ :

\section{Definition 31}

$$
\begin{aligned}
& A \varphi:=H \varphi \wedge \varphi \wedge G \varphi \text { (Always) } \\
& S \varphi:=\neg A \neg \varphi \text { (Sometimes) }
\end{aligned}
$$

According to Definition 31, it is always the case that $\varphi$ if and only if (i) $\varphi$ was the case in the past, (ii) $\varphi$ is the case, and (iii) $\varphi$ will always be the case in the future. Furthermore, it is sometimes the case that $\varphi$ just in case $\neg \varphi$ is not always the case.

Now, Let $\mathrm{L}_{\mathrm{E}}^{\mathrm{R}_{1}, \ldots, \mathrm{R}_{\mathrm{n}}}$ be a language just like $\mathrm{L}_{\mathrm{E}}$ except that its only mereological primitives are $R_{1}, \ldots, R_{n}$, for some natural number $n$ (where each $R_{i}$ is one of ' $<$ ',

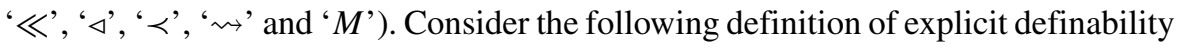
in the $E$-theory:

\section{Definition 32 (Explicit Definability)}

A notion expressed by a formula $\zeta$ containing as free variables the individual or second-order variables $v_{1}, \ldots, v_{n}$, is explicity definable in the E-theory solely in terms of the mereological primitives $R_{1}, \ldots, R_{n}$ if and only if there is some formula $\varphi$ of $\mathrm{L}_{\mathrm{E}}^{\mathrm{R}_{1}, \ldots, \mathrm{R}_{\mathrm{n}}}$, whose only free variables are $v_{1}, \ldots, v_{n}$, such that the following is an LE-truth:

$$
A \forall v_{1} \ldots \forall v_{n}\left(\varphi \leftrightarrow \zeta v_{1} \ldots v_{n}\right)
$$

Timeless parthood and immediate parthood are thus not definable in terms of parthood in the following sense:

\section{Theorem 2 (Undefinability of immediate parthood and timeless parthood in} terms of parthood) Timeless parthood and immediate parthood are not explicitly definable in terms of parthood in the E-theory.

We offer a proof of Theorem 2 in the appendix. We now turn to the positive result that all primitive mereological notions of $\mathrm{L}_{\mathrm{E}}$ are definable in the E-theory solely in terms of immediate parthood.

We begin by defining parthood as the ancestral of immediate parthood:

\section{Definition 33 (Parthood)}

Let $\sigma, \sigma_{1}, \sigma_{2}$ be any individual terms and $\zeta$ be any $n$-ary second-order term of $\mathrm{L}_{\mathrm{E}}$. Then:

- $\sigma_{1}<^{*} \sigma_{2}:=\forall Y\left(\left(\forall x\left(\sigma_{1} \ll x \rightarrow Y x\right) \wedge \forall z \forall w((Y z \wedge z \ll w) \rightarrow Y w)\right) \rightarrow Y \sigma_{2}\right)$;

$-\zeta<^{*} \sigma:=\forall Y\left((\forall x(\zeta \ll x \rightarrow Y x) \wedge \forall z \forall w((Y z \wedge z \ll w) \rightarrow Y w)) \rightarrow Y \sigma_{2}\right)$ 
That is, for $x$ to be a part of $y$ just is for $y$ to have all the immediate parthoodhereditary properties had by all objects of which $x$ is an immediate part.

Lemma 5 The following are $\mathrm{L}_{\mathrm{E}}$ truths:

1. $A \forall x \forall y\left(x<^{*} y \leftrightarrow x<y\right)$

2. $A \forall X \forall y\left(X<^{*} y \leftrightarrow X<y\right)$

Proof of Lemma 5 The proof of Lemma 5 is a straightforward consequence of the equivalence between the definitions of ancestral and transitive closure.

We now define what it is to be a principle of variable embodiment.

\section{Definition 34 (Principle of Variable Embodiment)}

Let $\sigma$ be any individual term and $\zeta$ be any monadic second-order term. Then:

$$
\zeta V^{*} \sigma:=\zeta \ll y \wedge S(\exists y(y \ll \sigma \wedge S \exists z(z \ll \sigma \wedge x \neq y)))
$$

That is, for a property $\mathscr{X}$ to be a principle of variable embodiment $y$ is for $\mathscr{X}$ to be a part of $y$ and for $y$ to have different immediate parts at different times.

Lemma 6 For every $\mathscr{M}, t \in \mathscr{T}$ and for every variable-assignment $g$ :

$\mathscr{M}, t, g[X / \mathscr{X}, y / y] \vDash X V^{*} y$ iff $y=\langle m, \mathscr{X}\rangle$ is a variable embodiment in $d_{\kappa, t}$, for some $m$.

\section{Proof of Lemma 6}

$\mathscr{M}, t, \mathscr{g}[X / \mathscr{X}, y / y] \vDash X V^{*} y$ if and only if (i) $\mathscr{X} \ll_{\mathscr{F}}^{t} y$, and (ii) $\exists t^{\prime} \in \mathscr{T}$, $x \in d_{\kappa, t^{\prime}}$ s. t. $x \ll_{\mathscr{F}}^{t^{\prime}} y$ and $\exists t^{\prime \prime} \in \mathscr{T}$, $\approx \in d_{\kappa, t^{\prime \prime}}$ s. t. $\approx \ll_{\mathscr{F}}^{t^{\prime \prime}} y$ and $\varkappa \neq x$, by Definition 28. Moreover, (i) is the case if and only if $y=\langle m, \mathscr{X}\rangle$ is an embodiment in $d_{\kappa, t}$, for some $m$, by Definition 9 , and (ii) is the case if and only if $y \in \mathscr{V}_{\kappa}$, by Definitions 9 and 6. So, $\mathscr{M}, t, g[X / \mathscr{X}, y / y] \vDash X V^{*} y$ if and only if $y=\langle m, \mathscr{X}\rangle$ is a variable embodiment in $d_{\kappa, t}$, for some $m$.

We will define timeless parthood in terms of the notions of immediate parthood and of being a rigid embodiment. The latter notion is defined as follows:

Definition 35 (Rigid Embodiment) Let $\sigma$ be any individual term. Then:

$R^{*} \sigma:=\exists x(x \ll \sigma) \wedge \neg \exists X^{1}\left(X^{1} \ll \sigma \wedge X^{1} V^{*} \sigma\right)$

That is, to be a rigid embodiment just is to have any immediate part and not to have a principle of variable embodiment as an immediate part.

Lemma 7 For every $\mathscr{M}, t \in \mathscr{T}$ and for every variable-assignment $q$ : $\mathscr{M}, t, g[x / x] \vDash R^{*} x$ iff $x$ is a rigid embodiment in $d_{\kappa, t}$. 
We are now in a position to define timeless parthood as the ancestral of immediate timeless parthood.

\section{Definition 36 (Timeless Parthood)}

Let $\sigma, \sigma_{1}, \sigma_{2}$ be any individual terms and $\zeta$ be any $n$-ary second-order term. Then:

- $\sigma_{1} \triangleleft^{*} \sigma_{2}:=\forall Y\left(\left(\forall x\left(\left(\sigma_{1} \ll^{*} x \wedge R^{*} x\right) \rightarrow Y x\right) \wedge \forall z \forall w\left(\left(Y z \wedge\left(z \ll^{*} w \wedge R^{*} z\right)\right) \rightarrow\right.\right.\right.$ $\left.Y w)) \rightarrow Y \sigma_{2}\right)$

$-\zeta \triangleleft^{*} \sigma:=\exists x\left(\zeta \ll^{*} x \wedge\left(x \triangleleft^{*} \sigma \vee x=\sigma\right)\right)$

Say that $x$ is an immediate rigid part of $y$ just in case $x$ is an immediate part of $y$ and $y$ is a rigid embodiment. Then, for $x$ to be a timeless part of $y$ just if for $y$ to have all the immediate and rigid-parthood hereditary properties had by all objects of which $x$ is an immediate and rigid part.

Lemma 8 The following are $\mathrm{L}_{\mathrm{E}}$-truths:

1. $A \forall x \forall y\left(x \triangleleft^{*} y \leftrightarrow x \triangleleft y\right)$;

2. $A \forall X \forall y\left(X \triangleleft^{*} y \leftrightarrow X \triangleleft y\right)$.

Proof of Lemma 8 As was the case with Lemma 5, Lemma 8 is a straightforward consequence of the equivalence between the definitions of ancestral and transitive closure.

We now turn to the definitions of manifestation and of temporary parthood.

\section{Definition 37 (Manifestation)}

Let $\sigma_{1}$ and $\sigma_{2}$ be any individual terms. Then:

$\sigma_{1} M^{*} \sigma_{2}:=\exists X\left(X V^{*} \sigma_{2} \wedge X \sigma_{1}\right)$

That is, for $x$ to be a manifestation of $y$ just is for $x$ to fall under $y$ 's principle of variable of embodiment.

Lemma 9 The following is an $\mathrm{L}_{\mathrm{E}-\text { truth: }}$

$A \forall x \forall y\left(x M^{*} y \leftrightarrow x M y\right)$.

Proof The explicit definability of manifestation is an immediate consequence of Definition 15, Definition 28 and Lemma 6.

Finally, temporary parthood is defined as expected in terms of parthood and manifestation.

\section{Definition 38 (Temporary Parthood)}

Let $\sigma, \sigma_{1}, \sigma_{2}$ be any individual terms of $\mathrm{L}_{\mathrm{E}}$ and $\zeta$ be any $n$-ary second-order term of LE. Then:

$-\sigma_{1} \prec^{*} \sigma_{2}:=\exists x \exists y\left(\sigma_{1} \leq^{*} x \wedge x M^{*} y \wedge y \leq^{*} \sigma_{2}\right)$; 
$-\zeta \prec^{*} \sigma:=\exists x \exists y\left(\zeta \leq^{*} x \wedge x M^{*} y \wedge y \leq^{*} \sigma_{2}\right)$

Lemma 10 The following are $\mathrm{L}_{\mathrm{E}}$ truths:

1. $A \forall x \forall y(x \prec * y \leftrightarrow x \prec y)$;

2. $A \forall X \forall y(X \prec * y \leftrightarrow X \prec y)$.

Proof of Lemma 10 Lemma 10 is a straightforward consequence of Lemmas 5 and 9 and Definition 28.

This last result also reveals that the notion of being mereologically chained is explicitly definable solely in terms of immediate parthood, given Lemma 1 .

Thus, as a corollary of Lemmas 5, 8, 9 and 10 we have that:

Theorem 3 (Definability of all mereological primitives in terms of immediate parthood) The notions of parthood, timeless parthood, temporary parthood, being mereologically chained and being a manifestation are all explicitly definable in terms of immediate parthood in the E-theory.

We now turn to the presentation of some mereological consequences of the Etheory.

\section{Mereological Results}

In this section we give some mereological results falling out of the formal semantics. These results should not be thought of entailments of Fine's postulates since we do not have anything like Completeness to go along with Soundness. There may well be other models of the theory of embodiments that fail to validate some of the results that follow.

Though there are many parthood notions available to consider, we focus on the main mereological notion of our semantics: parthood at $t$ given in Definition 12 as expressed by our binary predicate $<$ (Definition 28.7).

We already have some preliminary results for parthood at $t$ : Lemma 2 proves that this relation is a strict partial order (i.e. the E-theory counts as a Ground Mereology in the sense of Casati and Varzi [1, p. 36]). This is somewhat unremarkable, as most mereological theories meet this constraint. ${ }^{9}$

\subsection{Decomposition}

Here we address some questions about how embodiments are decomposed. A particular point of contention amongst hylomorphists regards the Weak Supplementation principle (WSP).

\footnotetext{
${ }^{9}$ Though there are exceptions; see e.g. Cotnoir and Bacon [4].
} 
WSP $\forall x \forall y(x<y \rightarrow \exists z(z<y \wedge \neg z \circ x))$

(Here $x \circ z$ is the mereological notion of overlap, defined as $\exists w(w \leqslant x \wedge w \leqslant z)$.) Many authors claim that Weak Supplementation is analytically true, indeed constitutive of the concept of proper parthood. ${ }^{10}$ Casati and Varzi [1, p. 39] go so far as to call any theory that satisfies the partial order axioms together with WSP 'Minimal Mereology'. By contrast Johnston [15] is a hylomorphist who rejects WSP.

WSP fails trivially here, due to the formulation using only the first-order quantifier. The theory of embodiments allows for distinct embodiments with exactly the same objects as material parts; $a / F \neq a / G$, where $F \neq G$ even though they aren't in the range of the first-order quantifier. It is natural then, to formulate more general versions of WSP as follows:

$\begin{array}{ll}\text { WSP 2a } & \forall x \forall y\left(x<y \rightarrow\left(\exists z(z<y \wedge \neg z \circ x) \vee \exists Z^{n}\left(Z^{n}<y \wedge \neg Z^{n} \circ x\right)\right)\right) \\ \text { WSP 2b } & \forall X^{m} \forall y\left(X^{m}<y \rightarrow\left(\exists z\left(z<y \wedge \neg z \circ X^{m}\right) \vee \exists Z^{n}\left(Z^{n}<y \wedge \neg Z^{n} \circ X^{m}\right)\right)\right.\end{array}$

Here the disjunctive consequent allows for parts of both first-order and second-order. (These principles require a similar generalization of the definition of $\circ$ ).

Koslicki [16, p. 155] presses the failures of Weak Supplementation as an objection to Fine's theory of embodiments. The worry is to do with variable embodiments and their manifestations. If the manifestation at $t$ of a variable embodiment is the only part-at- $t$ of that variable embodiment, then it looks as though parthood at $t$ will fail to satisfy WSP 2. Following Fine [13, p. 162], our formal semantics in Definition 9 treats the principle of a variable embodiment as a part at $t$ of that embodiment. We can easily respond to Koslicki's worry by proving that WSP 2 holds.

Theorem 4 (Weak Supplementation) For every model $\mathscr{M}$, time $t \in \mathscr{T}$ and variable-assignment $\mathcal{Q}$ :

$\mathscr{M}, t, q \vDash \forall x \forall y\left(x<y \rightarrow\left(\exists z(z<y \wedge \neg z \circ x) \vee \exists Z^{n}\left(Z^{n}<y \wedge \neg Z^{n} \circ x\right)\right)\right)$

$\mathscr{M}, t, q \vDash \forall X^{m} \forall y\left(X^{m}<y \rightarrow\left(\exists z\left(z<y \wedge \neg z \circ X^{m}\right) \vee \exists Z^{n}\left(Z^{n}<y \wedge \neg Z^{n} \circ X^{m}\right)\right)\right.$

To prove Theorem 4, we need a the following Lemma:

Lemma 11 Let $e=\left\langle m_{1}, \ldots m_{n}, \mathscr{X}\right\rangle$ be an embodiment in $\mathscr{B}_{\kappa} \cap\left(\mathscr{R}_{\kappa}^{n} \cup \mathscr{V}_{\kappa}\right)$, then for all $x$ s.t. $x$ is part of $e$ at $t, \mathscr{X}$ is not part of $x$ at $t$.

Proof of Lemma 11 Suppose $x<_{\mathscr{F}}^{t} \quad e$. Then $\exists n \in \mathbb{Z}^{+} \exists \vec{x}_{n}\left(\ll_{\mathscr{F}}^{t}\left(\vec{x}_{n}\right) \& x=\right.$ $x_{1} \& e=x_{n}$ ) by Definition 11. By Lemma 2, $x<_{\mathscr{F}}^{t} x_{n-1}$. Since $\mathscr{X}$ is simple (by construction), $x_{n-1} \neq X$ and $x_{n-1}=m_{j}(t)$ for some $j$ by Definition 3. Let ordinal $\alpha$ be the first stage such that $x \in \mathscr{B}_{\alpha}$. Since $x<<_{\mathscr{F}}^{t} m_{j}(t), m_{j}(t)$ must first appear in $\mathscr{B}_{\beta}$ for $\beta>\alpha$ (Definitions 9,11 ). But $\mathscr{X}$ is an $n$-ary relation (Definition 2) such that $\left\langle\ldots m_{j}(t) \ldots\right\rangle \in X(t)$ by Definition 7 . Hence $\mathscr{X}$ is an $n$-ary relation that first appears at stage $\beta$ by Definition 3. But since $\beta>\alpha, \mathscr{X}$ is not part of $x$ at $t$.

\footnotetext{
${ }^{10}$ Varzi [19, p. 110], Simons [18, p. 116, p. 26], Koslicki [17, p. 167-168], and Evnine [5, p. 58].
} 
Proof of Theorem 4 For WSP $2 \mathrm{a}$, assume for all $x, y \in d_{\kappa, t}, \mathscr{M}, t, q[x / x, y / y] \vDash$ $x<y$ [Definition 28.13]. Then $x<_{\mathscr{F}}^{t} y$ by Definition 28.7. By Definition $11 \exists n \in$ $\mathbb{Z}^{+} \exists \vec{x}_{n}\left(\ll_{\mathscr{F}}^{t}\left(\vec{x}_{n}\right) \& x=x_{1} \& y=x_{n}\right)$. By Definition $9, y=\left\langle m_{1} \ldots m_{m}, x\right\rangle$ and: either (i) $\exists i: 1 \leq i \leq m\left(x_{n-1}=m_{i}(t)\right) \& x_{n-1} \in d_{\kappa, t}$, or (ii) $x_{m-1}=\mathscr{X}$.

If (i) $x_{n-1}=m_{i}(t)$ we fix $g[Z / \mathscr{X}]$. Note that $\mathscr{X}<_{\mathscr{F}}^{t} y$ by Definitions 9 and 11 ; so $\mathscr{M}, t, \mathscr{q}\left[Z^{n} / \mathscr{X}\right] \vDash Z^{n}<y$. Note also, $\mathscr{X}$ is mereologically simple by construction, and so $\mathscr{X}$ and $x$ share a part at $t$ iff $\mathscr{X}<<_{\mathscr{F}}^{t} x$. By Lemma $11, \mathscr{X}$ is not part of $x$ at $t$, and hence have no parts in common. Hence $\mathscr{M}, t, q\left[Z^{n} / \mathscr{X}\right] \vDash$ $\neg Z^{n} \circ x$ by def. of $\circ$ and Definition 28.7. So $\mathscr{M}, t, q \vDash \exists Z^{n}\left(Z^{n}<y \wedge \neg Z^{n} \circ x\right)$ by Definitions 28.12 and 28.13.

If (ii) $x_{m-1}=\mathscr{X}$, either $m_{i}(t)=x$ for some $i$ or not. If so, we fix $g[Z / \mathscr{X}]$, and proceed as in case (i). If $m_{i}(t) \neq x$ for all $i$, there's some $e \in d_{\kappa, t}$ s.t. $x \ll_{\mathscr{F}}^{t} e$, and $e \leqslant_{\mathscr{F}}^{t} m_{i}(t)$ by Definitions 9 and 11. By Definition $9, e=\left\langle m_{1}^{e} \ldots m_{j}^{e}, \mathscr{X}\right\rangle$. Since $\mathscr{X}<_{\mathscr{F}}^{t} e \leqslant_{\mathscr{F}}^{t} m_{i}(t)<_{\mathscr{F}}^{t} y$, by Lemma $2 \mathscr{X}<_{\mathscr{F}}^{t} y$. Fix $g[Z / \mathscr{X}]$; then $\mathscr{M}, t, \mathscr{g}\left[Z^{n} / \mathscr{X}\right] \vDash Z^{n}<y$. Similarly, $\mathscr{X}$ has no parts in common with $x$ by Lemma 11. So $\mathscr{M}, t, g\left[Z^{n} / \mathscr{X}\right] \vDash \neg Z^{n} \circ x$, and so $\mathscr{M}, t, \mathscr{g} \vDash \exists Z^{n}\left(Z^{n}<\right.$ $\left.y \wedge \neg Z^{n} \circ x\right)$ ) by Definitions 28.12 and 28.13.

The proof of WSP $2 \mathrm{~b}$ is similar.

It is important to note that this result relies crucially on the assumption that any object that has properties or stands in relations must exist. Were it possible for $a$ to be $F$ without existing, there could be a rigid embodiment $a / F$ existing at $t$ (e.g. Aristotle qua the greatest philosopher of Antiquity) with only one part existing at $t$, namely $F$. These would be models that do not satisfy WSP $2 .{ }^{11}$

Are there any models of mereological gunk — objects with no atomic parts - or is the mereology atomistic? We begin by defining these notions.

Definition $39\left(\mathrm{~L}_{\mathrm{E}}\right.$ : Atoms) $A t(x):=\neg \exists y(y<x)$

Definition $40\left(\mathrm{~L}_{\mathrm{E}}\right.$ : Gunk) $G u(x):=\forall y(y<x \rightarrow \neg A t(y))$

Atomism $\forall x \exists y(y \leqslant x \wedge A t(y))$

Atomlessness $\forall x \exists y(y<x)$

Our formulation here does not quantify over formal parts of rigid or variable embodiments, since they are always mereologically simple by construction. Atomism requires that every object have a material part with no proper parts; and Atomlessness requires that every object have some material proper part.

\footnotetext{
${ }^{11}$ To get a better sense of what such a countermodel would look like: let $\mathscr{M}$ be such that $\mathscr{T}=\{1,2\}$ with $1 \leq 2, \mathscr{B}_{0}=d_{0,1}=\{a, b\}$ and $d_{0,2}=\{b\}, v(a)=a$ and $v(G)=\mathscr{G}$ s.t. $\mathscr{G}(1)=\mathscr{G}(2)=\{a\}$. Then:

$-\quad v^{q}(a / G)=\langle\lambda t \in \mathscr{T} . a, \mathscr{G}\rangle$

- $M, 2, g \vDash G<a / G$

- $\mathscr{M}, 2, \mathfrak{g} \not \models \exists z(z<a / G \wedge \neg z \circ G)$

- $\mathscr{M}, 2, \mathscr{g} \not \models \exists Z(Z<a / G \wedge \neg Z \circ G)$
} 
We can see intuitively that the mereology here satisfies Atomism, since all objects in the model are generated from the base-level entities $B_{0}$, which are assumed to be mereologically simple. ${ }^{12}$ We can actually show something stronger: parthood at $t$ is a well-founded partial order.

Lemma 12 For every time $t \in \mathscr{T}$, parthood at $t$ is well-founded in $\mathscr{B}_{\kappa} \cup \mathscr{D}_{\kappa}^{+}$.

Proof of Lemma 12 Let $\delta$ be an arbitrary nonempty subset of $\mathscr{B}_{\kappa} \cup \mathscr{D}_{\kappa}^{+}$and $\alpha$ be the least ordinal such that there is an $x \in \mathcal{S}$ such that $x \in \mathscr{B}_{\alpha} \cup \bigcup_{n \in \mathbb{N}} \mathscr{D}_{\alpha}^{n}$. Take any $x \in \mathcal{S} \cap\left(\mathscr{B}_{\alpha} \cup \bigcup_{n \in \mathbb{N}} \mathscr{D}_{\alpha}^{n}\right)$. Suppose, for reductio, that $\exists y \in \mathcal{S}\left(y<<_{\mathscr{F}}^{t} x\right)$. Then, there is an ordinal $\beta<\alpha$ such that $y \in \mathscr{B}_{\beta} \cup \bigcup_{n \in \mathbb{N}} \mathscr{D}_{\beta}^{n}$, by Definitions 3, 9 and 12, and so $\alpha$ is not the least ordinal such that there is an $x \in \mathcal{S}$ such that $x \in \mathscr{B}_{\alpha} \cup \bigcup_{n \in \mathbb{N}} \mathscr{D}_{\alpha}^{n}$. Contradiction. Hence, $\forall y \in \mathcal{S}: y \leq_{\mathscr{F}}^{t} x(y=x)$. Therefore, $\forall \mathcal{S} \subseteq \mathscr{B}_{\kappa} \cup \mathscr{D}_{\kappa}^{+}: \mathcal{S} \neq \emptyset\left(\exists x \in \mathcal{S}\left(\forall y \in \mathcal{S}: y \leq_{\mathscr{F}}^{t} x(y=x)\right)\right)$.

Thus, parthood at $t$ is well-founded in $\mathscr{B}_{\kappa} \cup \mathscr{D}_{\kappa}^{+}$.

This gives atomism as a clear corollary:

Theorem 5 For all $\mathscr{M}, t$, and $\mathcal{q}, \mathscr{M}, t, q \vDash \forall x \exists y(y \leqslant x \wedge A t(y))$.

Proof of Theorem 5 We show for arbitrary $q$ that $\mathscr{M}, t, q \vDash \exists y(y \leqslant x \wedge A t(y))$. By Lemma 12, every parthood at $t$ chain beginning at $\vartheta^{q}(x)$ has an <-minimal element; call it $y$. So $\mathscr{M}, t, q[y / y] \vDash y \leqslant x \wedge A t(y)$ by Definition 28.7. So $\mathscr{M}, t, q \vDash$ $\exists y(y \leqslant x \wedge A t(y))$ by Definition 28.13, and since $q$ was arbitrary we have the result.

In fact, Lemma 12 shows not only that the mereology is atomistic, it is also superatomistic in the sense of Cotnoir [2] — there is no infinite descent of parthood at all.

\subsection{Composition}

What about symmetric considerations involving composition? As should be clear enough, the theory is plenitudinous in that it permits mereological composition in a wide variety of cases. In classical mereology, fusion is defined in terms of parthood; but in Fine's theory, the operations of embodiment are not defined in terms of parthood in the usual way, and so principles regarding composition will be given directly about the operations of embodiment. (This is in keeping with Fine's [14] operationalism.)

\footnotetext{
${ }^{12}$ Again, we might supplement this framework following Fine's Segmentation approach, which would allow further decompositions of structural atoms into spatial parts corresponding to subregions. See Fine $[14$, p. 578]. Such an approach need not assume that objects are always segmented into spatial atoms, and so would be compatible with a gunky view.
} 
From this perspective, we can see that the regimented versions (R1) and (V1) are unrestricted composition principles. (R1) says that, given any things $\overrightarrow{\mathbf{y}}_{n}$ and relation $X^{n}$ holding of them, there is something (the rigid embodiment) that has those elements as its immediate parts. And we know from Theorem 1 that this principle holds in all models. It is worth noting another limitation which is merely an artefact of our choice of object language. In the case of rigid embodiments, the principle of embodiment is always an $n$-ary relation; because our language is finitary, the arity of any principle of embodiment can only ever be finite. It turns out, then, that a class of objects $C$ compose something if and only if $C$ is finite. ${ }^{13}$

Similarly, (V1) says that any individual concept has an embodiment existing (at $t$ ) iff it has a manifestation (at $t$ ), and again we know from Theorem 1 that this principle holds in all models. The constraint that a variable embodiment must have a manifestation might be thought of as a 'restriction' on composition; but it is no more a restriction than the corresponding unrestricted fusion principle in classical mereology, which permits only satisfiable conditions $\varphi$ to have fusions.

Mereological extensionality principles purport to show that objects that are equivalent with respect to some mereological property/relation are identical. One standard test of extensionality is called the 'Uniqueness of Composition', according to which taking the fusion of the same things always results in the same thing. Remember that the operations of embodiment are serving as our fusion operation, so one might be tempted to think that the regimented versions of (R3) and (V3) state (in the object language) the uniqueness of those modes of composition. However, that would be incorrect. They do not state that the result of applying the operation of rigid embodiment and variable embodiment to the same things (whether material parts under a relation or an individual concept), is always the same. After all, take some $a$ and $b$ and $R$ such that $R a b$ and $R b a$ both hold. Then $\langle a, b / R\rangle \neq\langle b, a / R\rangle$, and hence the operation of rigid embodiment is not unique on the same components - it is only unique on the same components in the same order, as stated in (R3).

What about so-called 'junk' - the dual of gunk? Is everything a proper part of something else? Or are there co-atoms - objects which are not proper parts of anything - in every model?

Definition $41\left(\mathrm{~L}_{\mathrm{E}}\right.$ : Junk) $J u(x):=\forall y(x<y \rightarrow \neg C o(y))$

Definition $42\left(\mathrm{~L}_{\mathrm{E}}\right.$ : Co-atoms) $\operatorname{Co}(x):=\neg \exists y(x<y)$

Co-atomism $\forall x \exists y(x \leqslant y \wedge \operatorname{Co}(y))$

Co-atomlessness $\forall x \exists y(x<y)$

Perhaps somewhat surprisingly, the mereology of embodiments satisfies coatomlessness.

Theorem 6 For all $\mathscr{M}, t$, and $q, \mathscr{M}, t, q \vDash \forall x \exists y(x<y)$.

\footnotetext{
${ }^{13}$ This restriction of composition, and its relation to 'junk', is discussed further in Cotnoir [3].
} 
Proof of Theorem 6 We show for arbitrary $\mathcal{g}$ that $\mathscr{M}, t, \mathcal{Z} \vDash \exists y(x<y)$. Call $v^{q}(x)=x$ and $x \in \mathscr{B}_{\kappa} \in \mathscr{M}$ by Definition 25. Then $x \in d_{0, t}$ or $x \in \mathscr{R}_{\kappa} \cup \mathscr{V}_{\kappa}$ by Definition 7. But $\mathscr{R}_{\kappa}=\bigcup_{j<\kappa} \mathscr{R}_{j}^{n}$ by Definition 4 and $\mathscr{V}_{\kappa}=\bigcup_{j<\kappa} \mathscr{V}_{j}$ by Definition 6. Hence in either case $x$ is some object appearing at some prior stage $i<\kappa$ (either an embodiment at some stage $j$, or base-level object appearing at stage 0 ). But then $x \in \mathscr{X}(t)$ for some $\mathscr{X} \in \mathscr{D}_{i}^{1}$ by Definition 2, and hence $\langle x, \mathscr{X}\rangle \in \mathscr{R}_{i+1} \cup \mathscr{V}_{i+1}$ by Definition 3. Call this embodiment $y$. Then $x<<_{\mathscr{F}}^{t} y$ by Definitions $9,11$. Hence $\mathscr{M}, t, \mathcal{Q}[y / y] \vDash x<y$ by Definition 28.7; and $\mathscr{M}, t, \mathcal{Q} \vDash \exists y(x<y)$ by Definition 28.13.

The universe of these models is 'junky' - it indefinitely extends from stage to stage. Not only is every model indefinitely extending, it is indefinitely extensible: for any model $\mathscr{M}_{\kappa}$, there is always another model $\mathscr{M}_{\mu}$ containing a higher 'cap', a limit ordinal $\mu>\kappa$, such that $\mathscr{B}_{\kappa}$ of $\mathscr{M}_{\mu}$ is identical to $\mathscr{B}_{\kappa}$ of $\mathscr{M}_{\kappa}$.

This fact fits neatly with the methodology that Fine [11,12] develops, called 'procedural postulationism' for mathematical entities. According to this view, the domain of the universal quantifier may always be extended to accommodate further objects, and the postulates of a given theory are imperatives for constructing a domain. In the case of mereology, Fine [13, p. 164] suggests that, while some objects are given, only those embodiments that are introduced via postulation will exist. The postulates of the theory of embodiments (R1)-(R6) and (V1)-(V6), then are instructions for introducing new individuals. In Fine [8, p. 103], Fine claims that rigid embodiments involving single individuals — 'qua objects' — are derivative entities; qualified individuals are posterior to the individuals which are qualified. The stages in our models give a definite content to that claim; here we understand the priority of stages not in terms of temporal precedence, nor in terms of ontological dependence, but rather they are to be understood postulationally. The mathematical structure of our models as constructed in stages, then, connects nicely with the underlying picture of ontological commitment.

\section{Primitive Ideology and Koslicki's Objections}

Aside from stabilizing the theory, and highlighting the various choice points for the overall philosophical view, our formal semantics serves another useful purpose: it provides an avenue of reply to one of the main objections that has been levelled against Fine's theory. Koslicki's [17] conception of objects shares a lot of commonalities to Fine's; they are both broadly neo-Aristotelian hylomorphists, who accept that forms are parts of the material objects they characterize. Koslicki proceeds with a single primitive notion of parthood for which she provides a theory, and she objects to Fine's theory for requiring multiple primitive notions of parthood. It is worth reproducing her objection here at length.

From a methodological point of view, Fine's analysis raises the worry that it leads to a proliferation of primitive, sui generis relations of parthood and composition. $[\ldots]$ the domain of ordinary material objects alone, in Fine's view, already calls 
for two distinct, primitive, sui generis relations of parthood and composition: the relations of composition by which rigid embodiments and variable embodiments are formed out of their respective components; as well as the relations of timeless part and temporary part that go along with these. [...]

Whatever connections there are between the two notions of composition and parthood thus do not follow from the general formal properties of the basic mereological vocabulary, independently of the domain of objects to which this vocabulary is currently applied; rather, they are explicitly imposed on these relations via postulates specifically tailored to the realm of ordinary material objects. Thus, even within this single domain of objects, Fine's strategy already leads on a (comparatively) small scale to a proliferation of distinct, primitive relations, which are not obviously needed in order to capture the conditions of existence, identity, location, character and part/whole structure of ordinary material objects. Since mereological vocabulary also applies outside of the realm of ordinary material objects, however, Fine's strategy would appear to lead to further distinct, primitive, sui generis relations of composition and parthood for each such domain of objects, accompanied by a system of postulates specifically tailored to the particular kinds of objects at issue. Such an approach takes on an overly stipulative and fractured air. $[17$, p. $82 \mathrm{f}]$

We can separate out two strands of Koslicki's complaint here: a main concern, and a subsidiary one. The main concern is that the notions of rigid and variable embodiments require distinct primitive notions of parthood, and hence a range of post hoc principles to bridge their interactions. This is, according to Koslicki, an objectionable proliferation of mereological ideology. The subsidiary concern is that these additional 'bridging' principles are not motivated by general considerations of formal mereology, but rather by domain-specific considerations of material constitution. ${ }^{14}$

As to the subsidiary concern, we think it is already sufficiently answered by Fine [10] who explicitly motivates rigid and variable embodiments by appeal not just to concrete material objects, but also to abstract objects. For example, he considers actions (p. 68), events (p. 72), musical works (p. 68), bodies of law (p. 72), word tokens and types (p. 68), tropes and universals (p. 68), sets (p. 73), propositions (p. 74 ), etc. Indeed, bringing the theory of material objects closer to theories of abstract objects was part of Fine's stated aim: "We are led, by these considerations, to a picture of the material world that has much more in common with the abstract realms of sets or of propositions than with the realms of concreta envisaged by the mereologist. (p. 74), 15

\footnotetext{
${ }^{14}$ See also Koslicki [16]

${ }^{15}$ In reply to Koslicki, Fine [13] writes:
} 
As to the main concern - that Fine's theory proliferates primitive sui generis parthood relations - the results of Section 6 reveal that, even though timeless parthood $(\triangleleft)$ and temporary parthood $(\prec)$ are not definable in terms of the natural notion of parthood defined as the ancestral of immediate parthood (Theorem 2), these notions are not primitive sui generis parthood relations; they are defined in a systematic way from the single general notion of immediate parthood $(\ll)$, as shown in Theorem 3. From the perspective of the model theory, Fine's theory requires exactly one primitive mereological relation. The ideology of this model-theoretic implementation of Fine's theory is no more onerous than Koslicki's own.

Acknowledgements This paper arose out of the weekly Metaphysics Research Group seminars at the Arché Philosophical Research Centre at the University of St Andrews. We are thankful to participants for their contributions. Thanks also to audience members at the the Metaphysics of Totality workshop and the University of Glasgow, especially Phillip Blum, Louis deRosset, Berta Grimau, Nathan Kirkwood, Anna-Sophia Maurin, Stephan Leuenberger, Alex Skiles, Naomi Thompson, Bruno Whittle, and Nathan Wildman. Thanks also to the Argument Clinic Group of the University of Lisbon, especially to Raimundo Henriques, José Mestre, Ricardo Miguel, and Diogo Santos. Thanks to two anonymous referees for this journal for their valuable comments. Special thanks to Gabriel Uzquiano and Maegan Fairchild for their comments on an earlier draft of this paper that led to many improvements. The research and writing of this paper was supported in part by a 2017-2018 Leverhulme Research Fellowship from the Leverhulme Trust.

Open Access This article is distributed under the terms of the Creative Commons Attribution 4.0 International License (http://creativecommons.org/licenses/by/4.0/), which permits unrestricted use, distribution, and reproduction in any medium, provided you give appropriate credit to the original author(s) and the source, provide a link to the Creative Commons license, and indicate if changes were made.

\section{Appendix}

\section{A.1 <-bisimulation}

In this appendix we offer a proof of Theorem 2. The proof of this theorem will appeal to a notion of model indistinguishability, <-bisimulation. We begin by formulating this notion and proving that it indeed captures a sense in which models are indistinguishable vis-á-vis parthood.

\section{Definition 43 ( $<$-Bisimulation)}

Where $\mathscr{M}=\left\langle\mathscr{T}, \mathscr{P}, d_{0}, \kappa, \leq, v\right\rangle$ and $\mathscr{M}^{\star}=\left\langle\mathscr{T} \star, \mathscr{P} \star, d_{0}^{\star}, \kappa^{\star}, \leq^{\star}, v^{\star}\right\rangle$ are any $\mathrm{L}_{\mathrm{E}}$ models (based, respectively, on frames $\mathscr{F}$ and $\mathscr{F} \star$ ), a function $\pi$ : $\mathscr{B}_{\kappa} \cup \bigcup_{n \in \mathbb{N}} \mathscr{D}_{\kappa}^{n} \cup \mathscr{T} \cup P \rightarrow \mathscr{B}_{\kappa}^{\star} \cup \bigcup_{n \in \mathbb{N}} \mathscr{D}_{\kappa}^{\star, n} \cup \mathscr{T} \star \cup P^{\star}$ is a <-bisimulation between $\mathscr{M}$ and $\mathscr{M}^{\star}, \mathscr{M} \stackrel{\pi}{\leftrightarrows} \mathscr{M}^{\star}$, if and only if:

1. For every $t \in \mathscr{T}: \pi\lceil\mathscr{T}$ is a bijection between $\mathscr{T}$ and $\mathscr{T} \star$;

2. For every $t, t^{\prime} \in \mathscr{T}: t \leq t^{\prime}$ if and only if $\pi(t) \leq \star \pi\left(t^{\prime}\right)$;

3. For every $p \in \mathscr{P}: \pi \uparrow \mathscr{P}$ is a bijection between $\mathscr{P}$ and $\mathscr{P} \star$;

4. For every $t \in \mathscr{T}: \pi \uparrow_{d_{\kappa, t}}$ is a bijection between $d_{\kappa, t}$ and $d_{\kappa, \pi(t)}^{\star}$;

5. For every $t \in \mathscr{T}$ and $p \in \mathscr{P}: \pi \Gamma_{d_{\kappa, t, p}}$ is a bijection between $d_{\kappa, t, p}$ and $d_{\kappa^{\star}, \pi(t), \pi(p)}^{\star}$ 
6. For every $n \in \mathbb{N}$ :

(a) $\pi \uparrow_{\mathscr{D}_{\kappa}^{\star}, n}$ is a bijection between $\mathscr{D}_{\kappa}^{n}$ and $\mathscr{D}_{\kappa^{\star}}^{\star, n}$;

(b) For every $\mathscr{X} \in \mathscr{D}_{\kappa}^{n}: \pi(\mathscr{X})(\pi(t))=\left\{\left\langle\pi\left(x_{1}\right), \ldots, \pi\left(x_{n}\right)\right\rangle\right.$ : $\left.\left\langle x_{1}, \ldots, x_{n}\right\rangle \in \mathscr{X}(t)\right\}$

7. For every individual constant $\sigma: \pi(\vartheta(\sigma))=u^{\star}(\sigma)$;

8. For every $n$-ary predicate $\zeta$, for every $n \in \mathbb{N}: \pi(v(\zeta))=v^{\star}(\zeta)$;

9. For every $t \in \mathscr{T}$ :

$$
\pi\left(<_{\mathscr{F}}^{t}\right)=\left\{\langle\pi(x), \pi(y)\rangle:\langle x, y\rangle \in<_{\mathscr{F}}^{t}\right\}=<_{\mathscr{F}}^{\pi(t)} .
$$

A first important result of the appendix is a theorem to the effect that $<$-bisimilar models are indiscernible vis-à-vis parthood, in the minimal sense that the same formulae of $\mathrm{L}_{\mathrm{E}}^{<}$are true in <-bisimilar models:

Theorem 7 For all models $\mathscr{M}$ and $\mathscr{M} \star$ (based, respectively, on frames $\mathscr{F}$ and $\mathscr{F} \star$ ) and function $\pi$ such that $\mathscr{M} \stackrel{\pi}{\leftrightarrows} \mathscr{M}^{\star}$, and for every formula $\varphi$ of $\mathrm{L}_{\mathrm{E}}^{<}$:

$\mathscr{M}, t, g \vDash \varphi$ if and only if $\mathscr{M}^{\star}, \pi(t), \pi(q) \vDash \varphi$,

The proof of this theorem will rely on some auxiliary definitions and lemmas. We start by defining the image of a variable-assignment under a bisimulation.

Definition 44 For all models $\mathscr{M}$ and $\mathscr{M}^{\star}$ (based, respectively, on frames $\mathscr{F}$ and $\mathscr{F} \star$ ), if $\mathscr{M} \stackrel{\pi}{\leftrightarrows} \mathscr{M}^{\star}$, then, for every variable-assignment $\mathcal{g}$ based on $\mathscr{F}, \pi(\mathscr{g})$ is that function such that, for every individual variable, second-order variable or spatial variable $\rho$,

$\pi(\mathscr{g})(\rho)=\pi(\mathscr{g}(\rho))$.

The following lemma shows that the image of a variable-assignment under a $<-$ bisimulation is itself a variable-assignment:

Lemma 13 For all models $\mathscr{M}$ and $\mathscr{M}^{\star}$ (based, respectively, on frames $\mathscr{F}$ and $\mathscr{F} \star$ ), if $\mathscr{M} \stackrel{\pi}{\leftrightarrows} \mathscr{M}^{\star}$, then $\pi(\mathscr{g})$ is a variable-assignment based on $\mathscr{F} \star$, for every variableassignment $\mathcal{Q}$ of $\mathscr{F}$.

Proof of Lemma 13 Suppose that $\mathscr{M} \stackrel{\pi}{\leftrightarrows} \mathscr{M}^{\star}$ where, $\mathscr{M}$ is based on $\mathscr{F}$ and $\mathscr{M}^{\star}$ is based on $\mathscr{F} \star$. Let $\mathcal{g}$ be an arbitrary variable-assignment based on $\mathscr{F}$.

Take an arbitrary individual variable $v$. We have that $\pi(\mathscr{g})(v)=\pi(\mathscr{g}(v))$. Since $g(v) \in \mathscr{B}_{\kappa}$, by Definition 26, it follows that $g(v) \in d_{\kappa, t}$, for some $t \in \mathscr{T}$, by Definition 7. So, $\pi(g)(v)=\pi(g(v)) \in d_{\kappa^{\star}, \pi(t)}^{\star}$, by Definition 43. Therefore, $\pi(g)(v) \in \mathscr{B}_{\kappa}^{\star}$, by Definition 7 .

Take an arbitrary $n$-ary second-order variable $V$. We have that $\pi(q)(V)=$ $\pi(g(V))$. Since $\mathscr{g}(V) \in \mathscr{D}_{\kappa}^{n}$, by Definition 26, it follows that $\pi(g)(V)=$ $\pi(g(V)) \in \mathscr{D}_{\kappa}^{\star}, n$, by Defn. 7 . 
Take an arbitrary spatial variable $s$. We have that $\pi(\mathscr{g})(s)=\pi(\mathscr{g}(s))$. Since $\mathscr{g}(s) \in \mathscr{P}$, by Definition 26, it follows that $\pi(\mathscr{g})(s)=\pi(\mathscr{g}(s)) \in \mathscr{P} \star$, by Definition 7.

Therefore, $\pi(\mathscr{g})$ is a variable-assignment based on $\mathscr{F} \star$.

The next lemma shows that values under variable-assignments are preserved under <-bisimulations:

Lemma 14 For all models $\mathscr{M}$ and $\mathscr{M}^{\star}$ (based, respectively, on frames $\mathscr{F}$ and $\mathscr{F} \star)$, if $\mathscr{M} \stackrel{\pi}{\leftrightarrows} \mathscr{M}^{\star}$, then, for every individual constant, individual variable, $n$-ary predicate or $n$-ary second-order variable $\sigma$ of $\mathrm{L}_{\mathrm{E}}^{<}$:

$\pi\left(u^{q}(\sigma)\right)=u^{\star}, \pi(q)(\sigma)$.

Proof of Lemma 14 Suppose $\sigma$ is an arbitrary individual constant or $n$-ary predicate. Then, $v(\sigma)=v^{q}(\sigma)$ and $v^{\star}(\sigma)=v^{\star}, \pi(q)(\sigma)$, by Definition 27. By Definition 43, $\pi(v(\sigma))=v^{\star}(\sigma)$. So, $\pi\left(u^{q}(\sigma)\right)=v^{\star}, \pi(q)(\sigma)$.

Suppose $\sigma$ is an individual variable or an $n$-ary second-order variable. Then, $q(\sigma)=v^{q}(\sigma)$, by Definition 27. By Lemma 13, $\pi(\mathscr{g})$ is a variable-assignment based on $\mathscr{F}^{\star}$. Furthermore, by Definition $27, \pi(\mathscr{g})(\sigma)=v^{\star}, \pi(g)(\sigma)$. Hence, $\pi\left(u^{q}(\sigma)\right)=v^{\star}, \pi(g)(\sigma)$.

So, either way, $\pi\left(v^{q}(\sigma)\right)=v^{\star}, \pi(q)(\sigma)$. This concludes the proof of Lemma 14 .

We now turn to the proof of Theorem 7:

Proof of Theorem 7 The proof is by induction on the complexity of a formula of $\mathrm{L}_{\mathrm{E}}^{<}$.

- $\varphi$ is $\zeta \sigma_{1} \ldots \sigma_{n}$ :

$\mathscr{M}, t, q \vDash \zeta \sigma_{1}, \ldots, \sigma_{n}$ iff $\left\langle v^{q}\left(\sigma_{1}\right), \ldots, v^{q}\left(\sigma_{n}\right)\right\rangle \in v^{q}(\zeta)(t)$, by Definition 28, iff $\left\langle\pi\left(v^{q}\left(\sigma_{1}\right)\right), \ldots, \pi\left(v^{q}\left(\sigma_{n}\right)\right)\right\rangle \in \pi\left(v^{q}(\zeta)\right)(\pi(t))$, by Definition 43, iff $\left.\left.\left.\left\langle v^{\star}, \pi(g)\left(\sigma_{1}\right)\right), \ldots, v^{\star, \pi(g)}\left(\sigma_{n}\right)\right)\right\rangle \in v^{\star, \pi(q)}(\zeta)\right)(\pi(t))$, by Lemma 14, iff $\mathscr{M}^{\star}, \pi(t), \pi(q) \vDash \zeta \sigma_{1} \ldots \sigma_{n}$, by Definition 28;

- $\varphi$ is $\operatorname{Loc}(s) \sigma$ :

$\mathscr{M}, t, q \vDash \operatorname{Loc}(s) \sigma$ iff $\vartheta^{q}(\sigma) \in d_{\kappa, t, q(s)}$, by Definition 28, iff $\pi\left(\vartheta^{q}(\sigma)\right) \in$ $\left\{\pi(x): x \in d_{\kappa, t, q(s)}\right\}$, by Definition 43, iff $v^{\star, \pi(g)}(\sigma) \in d_{\kappa^{\star}, \pi(t), \pi(g)(s)}^{\star}$, by Lemma 14, iff $\mathscr{M}^{\star}, \pi(t), \pi(g) \vDash \operatorname{Loc}(s) \sigma$, by Definition 28;

$-\varphi$ is $\sigma_{1}=\sigma_{2}$ :

$\mathscr{M}, t, \mathfrak{g} \vDash \sigma_{1}=\sigma_{2}$ iff $\vartheta^{q}\left(\sigma_{1}\right) \in d_{\kappa, t}$ and $u^{q}\left(\sigma_{1}\right)=v^{q}\left(\sigma_{2}\right)$, by Definition 28, iff $\pi\left(u^{q}\right)\left(\sigma_{1}\right) \in\left\{\pi(x): x \in d_{\kappa, t}\right\}$ and $\pi\left(u^{q}\left(\sigma_{1}\right)\right)=\pi\left(u^{q}\left(\sigma_{2}\right)\right)$, by Definition 43, iff $v^{\star}, \pi(g)(\sigma) \in d_{\kappa^{\star}, \pi(t)}^{\star}$ and $v^{\star}, \pi(g)\left(\sigma_{1}\right)=v^{\star, \pi(g)}\left(\sigma_{2}\right)$, by Lemma 14, iff $\mathscr{M}^{\star}, \pi(t), \pi(g) \vDash \sigma_{1}=\sigma_{2}$, by Definition 28;

$-\varphi$ is $\zeta_{1}=\zeta_{2}$ :

$\mathscr{M}, t, q \vDash \zeta_{1}=\zeta_{2}$ iff $v^{q}\left(\zeta_{1}\right)=v^{q}\left(\zeta_{2}\right)$, by Definition 28 , iff $\pi\left(v^{q}\left(\zeta_{1}\right)\right)=$ $\pi\left(v^{q}\left(\zeta_{2}\right)\right)$, by Definition 43 , iff $v^{\star}, \pi(g)\left(\zeta_{1}\right)=v^{\star}, \pi(g)\left(\zeta_{2}\right)$, by Lemma 14 , iff $\mathscr{M}^{\star}, \pi(t), \pi(g) \vDash \zeta_{1}=\zeta_{2}$, by Definition 28; 
$-\varphi$ is $\rho_{1}<\rho_{2}$ :

$\mathscr{M}, t, \mathcal{L} \vDash \rho_{1}<\rho_{2}$ iff $\boldsymbol{v}^{q}\left(\rho_{1}\right)$ and $\boldsymbol{v}^{q}\left(\rho_{2}\right)$ are defined and $\boldsymbol{v}^{q}\left(\rho_{1}\right)<_{\mathscr{F}}^{t}$ $u^{q}\left(\sigma_{2}\right)$, by Definition 28, iff $\pi\left(u^{q}\left(\rho_{1}\right)\right)$ and $\pi\left(u^{q}\left(\rho_{2}\right)\right)$ are defined and $\pi\left(u^{q}\left(\rho_{1}\right)\right)<<_{\mathscr{F}}^{\pi(t)} \pi\left(u^{q}\left(\rho_{2}\right)\right)$, by Definition 43, iff $u^{\star}, \pi(g)\left(\rho_{1}\right)$ and

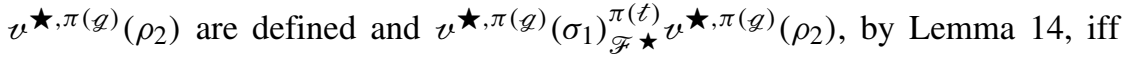
$\mathscr{M}^{\star}, \pi(t), \pi(q) \vDash \rho_{1}<\rho_{2}$, by Definition 28 ;

$-\varphi$ is $\neg \psi$ :

$\mathscr{M}, t, g \vDash \neg \psi$ iff $\mathscr{M}, t, q \not \forall \psi$, by Definition $28, \mathscr{M}, t, q \not \forall \psi$ iff $\mathscr{M}^{\star}, \pi(t), \pi(\mathscr{g}) \not \forall \psi$, by I.H., $\mathscr{M}^{\star}, \pi(t), \pi(\mathscr{g}) \not \forall \psi$ iff $\mathscr{M}^{\star}, \pi(t), \pi(\mathscr{g}) \vDash$ $\neg \psi$, by Definition 28;

$-\varphi$ is $\psi \wedge \chi$ :

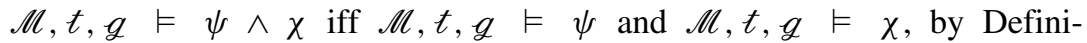
tion 28 , iff $\mathscr{M}^{\star}, \pi(t), \pi(q) \vDash \psi$ and $\mathscr{M}^{\star}, \pi(t), \pi(q) \vDash \chi$, by I.H., iff $\mathscr{M}^{\star}, \pi(t), \pi(q) \vDash \psi \wedge \chi$, by Definition 28;

- $\varphi$ is $\forall v \psi$ :

$\mathscr{M}, t, g \vDash \forall v \psi$ iff for all $x \in d_{\kappa, t}: \mathscr{M}, t, g[v / x] \vDash \psi$, by Definition 28, iff for all $x \in d_{\kappa, t}: \mathscr{M}^{\star}, \pi(t), \pi(\mathscr{g}[v / x]) \vDash \psi$, by I.H., iff for all $x \in d_{\kappa, t}$ : $\mathscr{M}^{\star}, \pi(t), \pi(q)[v / \pi(x)] \vDash \psi$, by Definition 44, iff for all $x \in d_{\kappa^{\star}, \pi(t)}^{\star}$ : $\mathscr{M}^{\star}, \pi(t), \pi(g)[v / x] \vDash \psi$, by Definition 43, iff $\mathscr{M}^{\star}, \pi(t), \pi(g) \vDash \forall v \psi$, by Definition 28;

- $\varphi$ is $\forall V \psi$ :

$\mathscr{M}, t, \mathscr{g} \vDash \forall V \psi$ iff for all $\mathscr{X} \in \mathscr{D}_{\kappa}^{n}: \mathscr{M}, t, \mathscr{g}[V / \mathscr{X}] \vDash \psi$, by Definition 28, iff for all $\mathscr{X} \in \mathscr{D}_{\kappa}^{n}: \mathscr{M}^{\star}, \pi(t), \pi(\mathscr{g}[V / \mathscr{X}]) \vDash \psi$, by I.H., iff for all $\mathscr{X} \in$ $\mathscr{D}_{\kappa}^{n}: \mathscr{M}^{\star}, \pi(t), \pi(\mathscr{Q})[V / \pi(\mathscr{X})] \vDash \psi$, by Definition 44, iff for all $\mathscr{X} \in \mathscr{D}_{\kappa^{\star}, n}^{\star}$ : $\mathscr{M}^{\star}, \pi(t), \pi(g) \vDash \psi[V / \mathscr{X}]$, by Definition 43, iff $\mathscr{M}^{\star}, \pi(t), \pi(\mathscr{g}) \vDash \forall V \psi$, by Definition 28;

- $\varphi$ is $\forall s \psi$ :

$\mathscr{M}, t, \mathscr{g} \vDash \forall s \psi$ iff for all $p \in \mathscr{P}: \mathscr{M}, t, q[s / p] \vDash \psi$, by Definition 28, iff for all $p \in \mathscr{P}: \mathscr{M}^{\star}, \pi(t), \pi(\mathscr{g}[s / p]) \vDash \psi$, by I.H., iff for all $p \in \mathscr{P}$ : $\left.\mathscr{M}^{\star}, \pi(t), \pi(g)[s / \pi(p)]\right) \vDash \psi$, by Definition 44 , iff for all $p \in \mathscr{P} \star$ : $\mathscr{M}^{\star}, \pi(t), \pi(g)[s / p] \vDash \psi$, by Definition 43, iff $\mathscr{M}^{\star}, \pi(t), \pi(q) \vDash \forall s \psi$, by Definition 28;

- $\varphi$ is $H \psi$ :

$\mathscr{M}, t, \mathscr{g} \vDash H \psi$ iff for all $t^{\prime} \in \mathscr{T}$ s. t. $t^{\prime} \leq t: \mathscr{M}, t^{\prime}, \mathscr{g} \vDash \psi$, by Definition 28, iff for all $t^{\prime} \in \mathscr{T}$ s. t. $t^{\prime} \leq t: \mathscr{M}^{\star}, \pi\left(t^{\prime}\right), \pi(g) \vDash \psi$, by I.H., iff for all $t^{\prime} \in \mathscr{T}$ s. t. $\pi\left(t^{\prime}\right) \leq \pi(t)$ : $\mathscr{M}^{\star}, \pi\left(t^{\prime}\right), \pi(\mathscr{g}) \vDash \psi$, by Definition 43 , iff for all $t^{\prime} \in \mathscr{T} \star$ s. t. $t^{\prime} \leq \pi(t): \mathscr{M}^{\star}, t^{\prime}, \pi(\mathscr{g}) \vDash \psi$, by Definition 43, iff $\mathscr{M}^{\star}, \pi(t), \pi(q) \vDash H \psi$, by Definition 28;

- $\varphi$ is $G \psi$ :

$\mathscr{M}, t, g \vDash G \psi$ iff for all $t^{\prime} \in \mathscr{T}$ s. t. $t \leq t^{\prime}: \mathscr{M}, t^{\prime}, \mathscr{g} \vDash \psi$, by Definition 28, iff for all $t^{\prime} \in \mathscr{T}$ s. t. $t \leq t^{\prime}: \mathscr{M}^{\star}, \pi\left(t^{\prime}\right), \pi(g) \vDash \psi$, by I.H., iff for all $t^{\prime} \in \mathscr{T}$ s. t. $\pi(t) \leq \pi\left(t^{\prime}\right): \mathscr{M}^{\star}, \pi\left(t^{\prime}\right), \pi(\mathscr{g}) \vDash \psi$, by Definition 43, iff for all $t^{\prime} \in \mathscr{T} \star$ s. t. $\pi(t) \leq t^{\prime}: \mathscr{M}^{\star}, t^{\prime}, \pi(q) \vDash \psi$, by Definition 43, iff $\mathscr{M}^{\star}, \pi(t), \pi(q) \vDash G \psi$, by Definition 28 . 
The <-bisimulation relation will be used to prove that timeless parthood and immediate parthood are not definable in the E-theory solely in terms of parthood. Our proof will rely on the following theorem:

Theorem 8 Suppose that there are models $\mathscr{M}$ and $\mathscr{M}^{\star}$ (based, respectively on frames $\mathscr{F}$ and $\mathscr{F} \star), t \in \mathscr{T}, x_{1}, \ldots, x_{n} \in d_{\kappa, t} \cup \bigcup_{n \in \mathbb{N}} \mathscr{D}_{\kappa}^{n}$ and $\pi$ such that:

1. $\mathscr{M} \stackrel{\pi}{\leftrightarrows} \mathscr{M}^{\star}$;

2. $\mathscr{M}, t, q\left[v_{1} / x_{1}, \ldots, v_{n} / x_{n}\right] \vDash \zeta v_{1} \ldots v_{n} ;$ and

3. $\mathscr{M}, \pi(t), \pi\left(g\left[v_{1} / x_{1}, \ldots, v_{n} / x_{n}\right]\right) \not \forall \zeta v_{1} \ldots v_{n}$.

Then, $\zeta$ is not explicitly definable in the E-theory solely in terms of $<$.

\section{Proof of Theorem 8}

Suppose that

(A) There are models $\mathscr{M}, \mathscr{M}^{\star}$, time $t \in \mathscr{T}_{\mathscr{M}}$, elements $x_{1}, \ldots, x_{n} \in d_{\mathscr{M}, \kappa, t} \cup$ $\bigcup_{n \in \mathbb{N}} \mathscr{D}_{\kappa}^{n}$, and a function $\pi$ such that:

(i) $\mathscr{M} \stackrel{\pi}{\leftrightarrows} \mathscr{M}^{\star}$;

(ii) $\mathscr{M}, t, q\left[v_{1} / x_{1}, \ldots, v_{n} / x_{n}\right] \vDash \zeta v_{1} \ldots v_{n}$; and

(iii) $\mathscr{M}^{\star}, \pi(t), \pi\left(\mathscr{g}\left[v_{1} / x_{1}, \ldots, v_{n} / x_{n}\right]\right) \not \forall \zeta v_{1} \ldots v_{n}$.

Suppose also, for reductio, that $\zeta$ is explicitly definable solely in terms of $<$. Then:

(B) There is some formula $\varphi$ of $\mathrm{L}_{\mathrm{E}}^{<}$(whose only free variables are $v_{1}, \ldots, v_{n}$ ) such that, for every model $\mathscr{M}: \vDash_{\mathscr{M}} A \forall v_{1} \ldots \forall v_{n}\left(\varphi \leftrightarrow \zeta v_{1} \ldots v_{n}\right)$.

From (B) it follows that:

(1) $\mathscr{M}, t, \mathscr{g}\left[v_{1} / x_{1}, \ldots, v_{n} / x_{n}\right] \vDash \quad \varphi$ iff $\mathscr{M}, t, \mathscr{g}\left[v_{1} / x_{1}, \ldots, v_{n} / x_{n}\right] \vDash$ $\zeta v_{1} \ldots v_{n} ;$ and

(2) $\mathscr{M}^{\star}, \pi(t), \pi\left(g\left[v_{1} / x_{1}, \ldots, v_{n} / x_{n}\right]\right) \quad \vDash \quad \varphi \quad$ iff $\mathscr{M}^{\star}, \pi(t), \pi\left(g\left[v_{1} / x_{1}, \ldots, v_{n} / x_{n}\right]\right) \vDash \zeta v_{1} \ldots v_{n}$.

Now, (i) and Theorem 7 imply that:

$\mathscr{M}, t, g\left[v_{1} / x_{1}, \ldots, v_{n} / x_{n}\right] \vDash \varphi$ iff $\mathscr{M}^{\star}, \pi(t), \pi\left(\mathscr{g}\left[v_{1} / x_{1}, \ldots, v_{n} / x_{n}\right]\right) \vDash \varphi$

The conjunction of this result with (1) and (2) implies that:

$\mathscr{M}, t, g\left[v_{1} / x_{1}, \ldots, v_{n} / x_{n}\right] \quad \vDash \quad \zeta v_{1} \ldots v_{n} \quad$ iff $\mathscr{M}^{\star}, \pi(t), \pi\left(\mathscr{g}\left[v_{1} / x_{1}, \ldots, v_{n} / x_{n}\right]\right) \vDash \zeta v_{1} \ldots v_{n}$

But this contradicts the conjunction of (ii) and (iii). Hence, the reductio assumption is false. $\zeta$ is not definable in the E-theory solely in terms of $<$. This concludes the proof of Theorem 8 .

\section{A.2 Undefinability of Timeless Parthood and Immediate Parthood}

Here's how we'll appeal to Theorem 8 to show that timeless parthood and immediate parthood are not definable in the E-theory solely in terms of parthood. We will begin by characterising a frame $\mathscr{F}=\left\langle\mathscr{T}, \mathscr{P}, d_{0}, \kappa, \leq\right\rangle$ and a model $\mathscr{M}$ based on $\mathscr{F}$. 
Then, we will define a bisimulation between $\mathscr{M}$ and itself. Finally, we will show that there is a time $t \in \mathscr{T}$, a variable-assignment $g$ based on $\mathscr{F}$ and objects $x$ and $y$ in $d_{\kappa, t}$ such that:

- $M, t, q[x / x, y / y] \vDash x \triangleleft y$, yet,

- $M, \pi(t), \pi(g[x / x, y / y]) \not \forall x \triangleleft y$,

and

- $M, t, \mathscr{g}[x / x, y / y] \vDash x \ll y$, yet,

- $\mathscr{M}, \pi(t), \pi(g[x / x, y / y]) \not \mid x \ll y$,

From Theorem 8 it will follow that none of timeless parthood and immediate parthood is definable in the E-theory solely in terms of parthood.

\section{A.2.1 The Model}

Our model will be based on the following frame:

\section{Definition 45}

Let $\mathscr{F}=\left\langle\mathscr{T}, \mathscr{P}, d_{0}, \kappa, \leq\right\rangle$, where:

- $\mathscr{T}=\{1,2\}$

- $1 \leq 2$;

$-\quad d_{0,1}=d_{0,2}=\{a, b\}$

- $\mathscr{P}=\{s\}$

$-\quad d_{0,1, s}=d_{0,2, s}=\{a, b\}$

- $\quad \kappa=\omega$.

The model $\mathscr{M}$ is defined as follows:

Definition 46 Let $\mathscr{M}=\left\langle\mathscr{T}, \mathscr{P}, d_{0}, \kappa, \leq, v\right\rangle$ where:

- $\quad v(\sigma)=a$, for every individual constant $\sigma$;

- For every $n \in \mathbb{N}$, every $n$-ary predicate $\zeta$, and every $t \in \mathscr{T}: \vartheta(\zeta)=\emptyset$.

Other models based on $\mathscr{F}$ would have been equally appropriate.

We will now distinguish a few elements definable in terms of frame $\mathscr{F}$ :

\section{Definition 47 Let:}

- $\quad \mathscr{G}_{a}^{a}$ and $\mathscr{G}_{a}^{b}$ be functions from $\mathscr{T}$ to $\wp\left(\mathscr{B}_{0}\right)$ such that:

- $\mathscr{G}_{b}^{a}(1)=\{a\}, \mathscr{G}_{a}^{a}(2)=\{b\}$;

- $\mathscr{G}_{a}^{b}(1)=\{b\}, \mathscr{G}_{a}^{b}(2)=\{a\}$.

- $\mathscr{q}_{a}^{a}$ and $\mathscr{q}_{a}^{b}$ be a functions from $\mathscr{T}$ to $\mathscr{B}_{0}$ such that:

$-q_{b}^{a}(1)=a, q_{b}^{a}(2)=b$;

- $q_{a}^{b}(1)=b, g_{a}^{b}(2)=a$; 
$-\quad e_{1}=\left\langle\mathscr{q}_{b}^{a}, \mathscr{G}_{b}^{a}\right\rangle$

$-\quad e_{2}=\left\langle q_{a}^{b}, \mathscr{G}_{a}^{b}\right\rangle$;

- $\mathscr{R}: \mathscr{T} \rightarrow \wp\left(\mathscr{B}_{1} \times \mathscr{B}_{1} \times \mathscr{B}_{1}\right)$ :

$-\mathscr{R}(1)=\mathscr{R}(2)=\left\{\left\langle a, e_{1}, e_{2}\right\rangle,\left\langle b, e_{1}, e_{2}\right\rangle\right\} ;$

$-e_{3}=\left\langle\lambda t \cdot a, \lambda t \cdot e_{1}, \lambda t \cdot e_{2}, \mathscr{R}\right\rangle$;

$-\quad e_{4}=\left\langle\lambda t . b, \lambda t \cdot e_{1}, \lambda t \cdot e_{2}, \mathscr{R}\right\rangle$.

We observe without proof that the following holds of the elements just characterised:

\section{Observation 1}

1. $e_{1} \in d_{1,1}$

7. $e_{2} \in d_{1,1,3}$

2. $e_{1} \in d_{1,2} \quad$ 8. $e_{2} \in d_{1,2,3}$

3. $e_{1} \in d_{1,1,3}$

9. $\mathscr{R} \in D_{1}^{3}$

15. $e_{3} \in d_{2,2,3}$

4. $e_{1} \in d_{1,2, s}$

10. $e_{3} \in d_{2,1}$

14. $e_{4} \in d_{2,1}$

5. $e_{2} \in d_{1,1}$

11. $e_{3} \in d_{2,2}$

15. $e_{4} \in d_{2,2}$

6. $e_{2} \in d_{1,2}$

12. $e_{3} \in d_{2,1, s}$

16. $e_{4} \in d_{2,1,3}$

17. $e_{4} \in d_{2,2,3}$

Observation $2 \forall x \in \mathscr{B}_{\kappa}, \forall t \in \mathscr{T}: x<_{\mathscr{F}}^{t} e_{3}$ iff $x<_{\mathscr{F}}^{t} e_{4}$.

The embodiments $e_{3}$ and $e_{4}$ will play a crucial role in showing the undefinability of timeless parthood and of immediate parthood in terms of parthood. As will be shown, $\mathscr{M}$ is $<$-bisimilar to itself via a $<$-bisimulation $\pi$ that maps a to itself and maps $e_{3}$ to $e_{4}$. Yet, $a$ is a timeless part of $e_{3}$, even though $a$ is not a timeless part of $e_{4}$. Similarly, $a$ is an immediate part of $e_{3}$, even though $a$ is not an immediate part of $e_{4}$. In conjunction with Theorem 8 , this result shows that timeless parthood and immediate parthood are not definable in terms of parthood. We now turn to the definition of the <-bisimulation $\pi$.

\section{A.2.2 The <-bisimulation}

We begin by defining the set of all partial functions, $\mathscr{H}_{i}$, from $\mathscr{T}$ to $\mathscr{B}_{i}$, for each $i$ such that $0 \leq i<\kappa$ :

Definition 48 For each $i$ such that $0 \leq i<\kappa$, let:

$-\mathscr{H}_{i}=\left\{h: \mathscr{T} \rightarrow \mathscr{B}_{i}\right\}$

- $\mathscr{H}_{\kappa}=\bigcup_{i<\kappa} \mathscr{H}_{i}$.

The <-bisimulation $\pi$ will be defined in terms of the following function between entities defined in terms of the frame $\mathscr{F}$ :

\section{Definition 49 ( $\theta$-Function)}

Let $\theta$ be a function with domain $\mathscr{B}_{\kappa} \cup \mathscr{D}_{\kappa}^{+} \cup \mathscr{H}_{\kappa}$ simultaneously defined as follows: 
1. $\forall x \in \mathscr{B}_{\kappa}$ :

(a) If $x \in \mathscr{B}_{0}$, then $\theta(x)=x$;

(b) If $x \notin \mathscr{B}_{0}$, then:

(i) if $x \neq e_{3}$ and $x \neq e_{4}$, then

$$
\begin{aligned}
& \theta(x)=\left\langle\theta\left(m_{1}\right), \ldots, \theta\left(m_{n}\right), \theta(\mathscr{X})\right\rangle, \\
& \text { where } x=\left\langle m_{1}, \ldots, m_{n}, \mathscr{X}\right\rangle ;
\end{aligned}
$$

(ii) if $x=e_{3}$, then $\theta(x)=e_{4}$;

(iii) if $x=e_{4}$, then $\theta(x)=e_{3}$;

2. $\forall \mathscr{X} \in \mathscr{D}_{\kappa}, t \in \mathscr{T}:$

$$
\theta(\mathscr{X})(t)=\left\{\left\langle\theta\left(x_{1}\right), \ldots, \theta\left(x_{n}\right)\right\rangle:\left\langle x_{1}, \ldots, x_{n}\right\rangle \in \mathscr{X}(t)\right\} ;
$$

3. $\forall h \in \mathscr{H}_{\kappa}: \forall t \in \mathscr{T}, \theta(h)(t)=\theta(h(t))$.

We now show that relevant restrictions of $\theta$ turn out to be bijections:

Lemma 15 (Bijections) For every $i<\kappa$ and $t \in \mathscr{T}$ and $n \in \mathbb{N}$ :

1. $\theta \uparrow_{d_{i, t}}$ is a bijection from $d_{i, t}$ to $d_{i, t}$;

2. $\theta \uparrow_{d_{i, t, s}}$ is a bijection from $d_{i, t, s}$ to $d_{i, t, s}$;

3. $\theta \Gamma_{\mathscr{D}_{i}^{n}}^{n}$ is a bijection from $\mathscr{D}_{i}^{n}$ to $\mathscr{D}_{i}^{n}$;

4. $\theta\left\lceil\mathscr{H}_{i}\right.$ is a bijection from $\mathscr{H}_{i}$ to $\mathscr{H}_{i}$.

\section{Proof of Lemma A.2.2}

1.(a) $\left(\forall x \in d_{i, t}: \theta(x) \in d_{i, t}\right)$ :

Suppose, for reductio, that there is a least ordinal $i<\kappa$ such that $\theta(x) \notin d_{i, t}$, for some $t \in \mathscr{T}$ and $x \in d_{i, t}$. Clearly, $x \neq a, x \neq b, x \neq e_{3}$ and $x \neq e_{4}$. So, $i$ is a successor ordinal $j+1, x=\left\langle m_{1}, \ldots, m_{n}, \mathscr{X}\right\rangle$, by Definition 7 , and $\theta(x)=\left\langle\theta\left(m_{1}\right), \ldots, \theta\left(m_{n}\right), \theta(\mathscr{X})\right\rangle$, by Definition 49 .

Suppose $x$ is an $n$-ary rigid embodiment. Then: (i) $\theta\left(m_{l}\right)$ is a constant function from $\mathscr{T}$ to $\mathscr{B}_{j}$, for each $1 \leq l \leq n$, by Definitions 4 , 49 and the reductio assumption; (ii) $\theta(\mathscr{X}) \in \mathscr{D}_{j}^{n}$, by Definitions 4, 49 and the reductio assumption; and (iii) $\left\langle m_{1}(t), \ldots, m_{n}(t)\right\rangle \in \mathscr{X}(t)$ if and only if $\left\langle\theta\left(m_{1}\right)(t), \ldots, \theta\left(m_{n}\right)(t)\right\rangle \in \theta(\mathscr{X})(t)$, by Definition 49 . But $\left\langle m_{1}(t), \ldots, m_{n}(t)\right\rangle \in \mathscr{X}(t)$, since $x \in d_{j+1, t}$, by Definition 7. So, $\left\langle\theta\left(m_{1}\right)(t), \ldots, \theta\left(m_{n}\right)(t)\right\rangle \in \theta(\mathscr{X})(t)$. So, $\theta(x)$ is an $n$-ary rigid embodiment in $d_{j+1, t}$, by Definitions 4 and 7 . But this contradicts the reductio assumption.

Suppose instead that $x$ is a variable embodiment. Then: (i) $\theta(\mathscr{X})$ is an individual concept in $\mathscr{D}_{j}^{1}$, by Definitions 6, 49 and the reductio assumption; (ii) $\theta\left(m_{1}\right) \in \mathscr{H}_{j}$, by Definition 6, Definition 49 and the reductio assumption; (iii) $\theta(\mathscr{X})(t)=\left\{\theta\left(m_{1}\right)(t)\right\}$ if $\theta\left(m_{1}\right)(t)$ is defined and otherwise $\theta(\mathscr{X})(t)=\emptyset$, for every $t \in \mathscr{T}$, by Definition 6 , Definition 49 and the reductio assumption; (iv) $m_{1}(t) \in \mathscr{X}(t)$ if and only if $\theta\left(m_{1}\right)(t) \in \theta(\mathscr{X})(t)$, by Definition 49. But $m_{1}(t) \in \mathscr{X}(t)$, since $x \in d_{j+1, t}$, by Definition 7 . 
So, $\theta\left(m_{1}\right)(t) \in \theta(\mathscr{X})(t)$. So, $\theta(x)$ is a variable embodiment in $d_{j+1, t}$, by Definitions 6 and 7. But this contradicts the reductio assumption.

Therefore, the reductio assumption is false. Hence, for every $i<\kappa$ and $t \in \mathscr{T}: \theta(x) \in d_{i, t}$, for every $x \in d_{i, t}$;

1.(b) $\left(\forall x, y \in d_{i, t}: \theta(x)=\theta(y) \Rightarrow x=y\right)$ :

Suppose, for reductio, that there is a least ordinal $i<\kappa$ such that $\theta(x)=$ $\theta(y)$ and $x \neq y$, for some $x, y$ in $d_{i, t}$ and $t \in \mathscr{T}$. Clearly, $i$ is a successor ordinal $j+1$, where $j \geq 2$. So, by the reductio assumption, and Definitions 7 and 49 , there are $x=\left\langle m_{1}^{x}, \ldots, m_{n}^{x}, \mathscr{X}\right\rangle \in d_{j+1, t}$ and $y=\left\langle m_{1}^{y}, \ldots, m_{n}^{y}, x\right\rangle \in d_{j+1, t}$ such that $x \neq y$ and $\theta(x)=$ $\left\langle\theta\left(m_{1}^{x}\right), \ldots, \theta\left(m_{n}^{x}\right), \theta(\mathscr{X})\right\rangle=\theta(y)=\left\langle\theta\left(m_{1}^{y}\right), \ldots, \theta\left(m_{n}^{y}\right), \theta(\mathscr{y})\right\rangle$. Вy the reductio assumption and Definition 49, $\theta\left(m_{l}^{x}\right)=\theta\left(m_{l}^{y}\right)$, for every $l$ such that $1 \leq l \leq n$, and $\theta(\mathscr{X})=\theta(\mathscr{Y})$. But then, $\theta(x)=\theta(y)$. Yet, this contradicts the reductio assumption. So, for every ordinal $i<\kappa$ and $t \in \mathscr{T}$ : $\forall x, y \in d_{i, t}: \theta(x)=\theta(y) \Rightarrow x=y$;

1.(c) $\left(\forall y \in d_{i, t} \exists x \in d_{i, t}: \theta(x)=y\right)$ :

Suppose, for reductio, that there is a least ordinal $i<\kappa$ and a $y \in d_{i, t}$ such that for every $x \in d_{i, t}, \theta(x) \neq y$. Clearly, $i$ is a successor ordinal $j+1$, where $j \geq 2$. So, $y=\left\langle m_{1}^{y}, \ldots, m_{n}^{y}, y\right\rangle$, by Definition 7 . Now, by the reductio assumption, there are $m_{1}^{x}, \ldots, m_{n}^{x}$ and $\mathscr{X}$ such that $m_{1}^{y}=\theta\left(m_{1}^{x}\right)$, $\ldots, m_{n}^{y}=\theta\left(m_{n}^{x}\right)$ and $\mathcal{Y}=\theta(\mathscr{X})$. Let $x=\left\langle m_{1}^{x}, \ldots, m_{n}^{x}, \mathscr{X}\right\rangle$. Suppose $y$ is a rigid embodiment in $d_{j+1, t}$. Then, for every $l$ such that $1 \leq l \leq n, m_{l}^{x}$ is a constant function from $\mathscr{T}$ to $\mathscr{B}_{j}, \mathscr{X} \in \mathscr{D}_{j}^{n}$ and $\left\langle m_{1}^{x}(t), \ldots, m_{n}^{x}(t)\right\rangle \in$ $\mathscr{X}(t)$, by the reductio assumption and Definitions 4,7 and 49 . So, $x$ is a rigid embodiment in $d_{j+1, t}$, by Definitions 4 and 7. Suppose instead that $y$ is a variable embodiment in $d_{j+1, t}$. Then, $\mathscr{X}$ is an individual concept in $\mathscr{D}_{j}^{1}$, $m_{1}^{x} \in \mathscr{H}_{j}, \forall t \in \mathscr{T}: m_{1}(t)$ is defined if and only if $\mathscr{X}(t)=\left\{m_{1}(t)\right\}$, and $m_{1}^{x}(t) \in \mathscr{X}(t)$, by the reductio assumption and Definitions 6, 7 and 49. So, $x$ is a variable embodiment in $d_{j+1, t}$. Either way, $\theta(x)=y$, where $x \in$ $d_{j+1, t}$. But this contradicts the reductio assumption. So, for every ordinal $i<\kappa$ and $t \in \mathscr{T}, \forall y \in d_{i, t} \exists x \in d_{i, t}: \theta(x)=y$.

Therefore, for every ordinal $i<\kappa$ and $t \in \mathscr{T}, \theta \uparrow_{i, t}$ is a bijection from $d_{i, t}$ to $d_{i, t}$.

2.(a) $\left(\forall x \in d_{i, t, j}: \theta(x) \in d_{i, t, s}\right)$ :

Suppose, for reductio, that there is a least ordinal $i$ such that $\theta(x) \notin d_{i, t, s}$, for some $x \in d_{i, t, s}$ and $t \in \mathscr{T}$. Clearly, $x \neq a, x \neq b, x \neq e_{3}$ and $x \neq$ $e_{4}$. So, $i$ is a successor ordinal $j+1, x=\left\langle m_{1}, \ldots, m_{n}, \mathscr{X}\right\rangle$, by Definition 8 , and $\theta(x)=\left\langle\theta\left(m_{1}\right), \ldots, \theta\left(m_{n}\right), \theta(\mathscr{X})\right\rangle$, by Definition 49. Since $x \in d_{i, t, s}$, there is some $m_{l}$ such that $m_{l}(t) \in d_{j, t, s}$, by Definition 8 . So, by the reductio assumption and Definition 49, there is some $l$ such that $1 \leq l \leq n$ and $\theta\left(m_{l}(t)\right)=\theta\left(m_{l}\right)(t) \in d_{j, t, s}$. But then, $\theta(x) \in d_{j+1, t, s}=d_{i, t, s}$, by Definition 8. This contradicts the reductio assumption. Therefore, the reductio assumption is false: for every $i<\kappa$ and $t \in \mathscr{T}, \forall x \in d_{i, t, s}: \theta(x) \in d_{i, t, s}$.

2.(b) $\left(\forall x, y \in d_{i, t, s}: \theta(x)=\theta(y) \Rightarrow x=y\right)$ :

Suppose, for reductio, that there is a least ordinal $i<\kappa$ and such that $\theta(x)=$ $\theta(y)$ and yet $x \neq y$, where $x$ and $y$ belong to $d_{i, t, s}$. Hence, there are $x$ 
and $y \in d_{i, t}$ such that $\theta(x)=\theta(y)$, by Definition 8 and yet $x \neq y$. But this contradicts the claim that $\theta \uparrow_{i, t}$ is a bijection from $d_{i, t}$ to $d_{i, t}$. Contradiction. So, the reductio assumption is false: for every ordinal $i<\kappa$ and $t \in \mathscr{T}, \forall x, y \in d_{i, t, s}: \theta(x)=\theta(y) \Rightarrow x=y$.

2.(c) $\left(\forall y \in d_{i, t, j} \exists x \in d_{i, t, s}: \theta(x)=y\right)$ :

Suppose, for reductio, that there is a least $i<\kappa$ and a $y \in d_{i, t, 3}$ such that, for every $x \in d_{i, t, s}, \theta(x) \neq y$. Clearly, $i$ is a successor ordinal $j+1$, where $j \geq 2$. So, $y=\left\langle m_{1}^{y}, \ldots, m_{n}^{y}, y\right\rangle$, by Definition 8 . So, there is an $x=\left\langle m_{1}^{x}, \ldots, m_{n}^{x}, \mathscr{X}\right\rangle \in d_{j+1, t}$ such that $\theta(x)=y$, since $\theta \uparrow_{i, t}$ is a bijection and $y \in d_{j+1, t}$, by Definition 8. Now, from the reductio assumption and Definition 49 it follows that, for every $l$ such that $1 \leq l \leq n$, $m_{l}^{y} \in d_{j, t, s}$ if and only if $m_{l}^{x} \in d_{j, t, s}$. But there is some $l$ such that $m_{l}^{y}(t) \in d_{j, t, s}$, by Definition 8 , since $y \in d_{j+1, t, s}$. Therefore, there is some $l$ such that $m_{l}^{x}(t) \in d_{j, t, s}$. But then, $x \in d_{j+1, t, s}$, by Definition 8 . This contradicts the reductio assumption. So, for every ordinal $i<\kappa, t \in \mathscr{T}$ $\forall y \in d_{i, t, s} \exists x \in d_{i, t, s}: \theta(x)=y$.

Therefore, for every ordinal $i<\kappa$ and $t \in \mathscr{T}, \theta \uparrow_{d_{i, t, s}}$ is a bijection from $d_{i, t, s}$ to $d_{i, t, s}$.

3. The claim that for every $i<\kappa, \theta \Gamma_{\mathscr{D}_{i}^{n}}$ is a bijection from $\mathscr{D}_{i}^{n}$ to $\mathscr{D}_{i}^{n}$ is a straightforward consequence of the fact that for every $i<\kappa$ and $t \in \mathscr{T}$, $\theta \uparrow_{i, t}$ is a bijection from $d_{i, t}$ to $d_{i, t}$;

4. The claim that for every $i<\kappa, \theta\left\lceil\mathscr{H}_{i}\right.$ is a bijection from $\mathscr{H}_{i}$ to $\mathscr{H}_{i}$ is a straightforward consequence of the fact that for every $i<\kappa$ and $t \in \mathscr{T}$, $\theta \uparrow d_{i, t}$ is a bijection from $d_{i, t}$ to $d_{i, t}$.

Our final result about the $\theta$ function concerns the preservation of parthood under the $\theta$ function:

Lemma 16 For every $x, y \in d_{\kappa, t} \cup \mathscr{D}_{\kappa}$, for every $t \in \mathscr{T}: x<_{\mathscr{F}}^{t} y$ if and only if $\theta(x)<_{\mathscr{F}}^{t} \theta(y)$.

\section{Proof of Lemma 16}

$(\Rightarrow)$ : Suppose, for reductio, that there are $x, y \in d_{\kappa, t} \cup \mathscr{D}_{\kappa}$ such that $x<_{\mathscr{F}}^{t} y$ and $\theta(x) \nless_{\mathscr{F}}^{t} \theta(y)$, for some $t \in \mathscr{T}$. Then, there is a least ordinal $i$ such that $x<_{\mathscr{F}}^{t} y$ and $\theta(x) \nless_{\mathscr{F}}^{t} \theta(y)$, for some $x, y \in d_{i, t} \cup \bigcup_{n \in \mathbb{N}} \bigcup_{n \in \mathbb{N}} \mathscr{D}_{i}^{n}$ and $t \in \mathscr{T}$. Now, $i$ is a successor ordinal $j+1$, where $j>2$, since $e_{3}$ and $e_{4}$ share the same parts, by Observation 2 , and $\theta(y)=y$ for every $y \in \mathscr{B}_{2}$ such that $y \neq e_{3}$ and $y \neq e_{4}$.

So, $y=\left\langle m_{1}^{y}, \ldots, m_{n}^{y}, y\right\rangle$, by Definition 7. Now, either $x=\mathscr{y}$, or else $x \leq_{\mathscr{F}}^{t} m_{l}^{y}(t)$, for some $l$ such that $1 \leq l \leq n$. If $x=\mathscr{Y}$, then, $x<_{\mathscr{F}}^{t} y$. But then, $\theta(x)=\theta(y)<_{\mathscr{F}}^{t} \theta(y)=\left\langle\theta\left(m_{1}^{y}\right), \ldots, \theta\left(m_{n}^{y}\right), \theta(\mathscr{y})\right\rangle$. So, suppose that $x \leq_{\mathscr{F}}^{t} m_{l}^{y}(t)$. If $x=m_{l}^{y}(t)$, then $\theta(x)=\theta\left(m_{l}^{y}(t)\right)=\theta\left(m_{l}^{y}\right)(t)=$ $\left\langle\theta\left(m_{1}^{y}\right), \ldots, \theta\left(m_{n}^{y}\right), \theta(\mathscr{Y})\right\rangle$. So, suppose instead that $x<_{\mathscr{F}}^{t} m_{l}^{y}(t)$. Then, by the reductio assumption, $\theta(x)<_{\mathscr{F}}^{t} \theta\left(m_{l}^{y}\right)(t)$. But $\theta\left(m_{l}^{y}\right)(t)<_{\mathscr{F}}^{t} \theta(y)=$ $\left\langle\theta\left(m_{1}^{y}\right), \ldots, \theta\left(m_{n}^{y}\right), \theta(\mathscr{Y})\right\rangle$. Hence, $\theta(x)<_{\mathscr{F}}^{t} \theta(y)$, by the Definition of $<_{\mathscr{F}}^{t}$. 
But this contradicts the reductio assumption. Therefore, for every $x, y \in d_{\kappa, t} \cup$ $\mathscr{D}_{\kappa}$, for every $t \in \mathscr{T}: x<_{\mathscr{F}}^{t} y$ only if $\theta(x)<_{\mathscr{F}}^{t} \theta(y)$.

$(\Leftarrow)$ : Suppose, for reductio, that there are $x, y \in d_{\kappa, t} \cup \mathscr{D}_{\kappa}$ such that $x \nless_{\mathscr{F}}^{t} y$ and $\theta(x)<_{\mathscr{F}}^{t} \theta(y)$, for some $t \in \mathscr{T}$. Then, there is a least ordinal $i$ such that $x \nless_{\mathscr{F}}^{t} y$ and $\theta(x)<_{\mathscr{F}}^{t} \theta(y)$, for some $x, y \in d_{i, t} \cup \bigcup_{n \in \mathbb{N}} \bigcup_{n \in \mathbb{N}} \mathscr{D}_{i}^{n}$ and $t \in \mathscr{T}$. Now, $i$ is a successor ordinal $j+1$, where $j>2$, since $e_{3}$ and $e_{4}$ share the same parts, by Observation 2 , and $\theta(y)=y$ for every $y \in \mathscr{B}_{2}$ such that $y \neq e_{3}$ and $y \neq e_{4}$.

So, $y=\left\langle m_{1}^{y}, \ldots, m_{n}^{y}, y\right\rangle$, by Definition 7 , and $\theta(y)=$ $\left\langle\theta\left(m_{1}^{y}\right), \ldots, \theta\left(m_{n}^{y}\right), \theta(\mathscr{Y})\right\rangle$. Now, either $\theta(x)=\theta(\mathscr{Y})$, or else $\theta(x) \leq_{\mathscr{F}}^{t}$ $\theta\left(m_{l}^{y}\right)(t)=\theta\left(m_{l}^{y}(t)\right)$, for some $l$ such that $1 \leq l \leq n$. If $\theta(x)=\theta(\mathcal{Y})$, then, $x=\mathscr{Y}$, since $\theta \Gamma_{\mathscr{D}_{j}^{n}}^{n}$ is a bijection between $\mathscr{D}_{j}^{n}$ and $\mathscr{D}_{j}^{n}$. But then, clearly, $x=y<_{\mathscr{F}}^{t}\left\langle m_{1}^{\frac{j}{y}}, \ldots, m_{n}^{y}, \mathscr{y}\right\rangle$. Suppose $\theta(x) \leq_{\mathscr{F}}^{t} \theta\left(m_{l}^{y}\right)(t)$. If $\theta(x)=\theta\left(m_{l}^{y}(t)\right)$, then $x=m_{l}^{y}(t)$, since $\theta \Gamma_{d_{j, t}}$ is a bijection between $d_{j, t}$ and $d_{j, t}$. So, $x=m_{l}^{y}(t)<_{\mathscr{F}}^{t}\left\langle m_{1}^{y}, \ldots, m_{n}^{y}, y\right\rangle$. So, suppose that $\theta(x)<_{\mathscr{F}}^{t} \theta\left(m_{l}^{y}\right)(t)$. Then, by the reductio assumption, $x<_{\mathscr{F}}^{t} m_{l}^{y}(t)$. But $m_{l}^{y}(t)<_{\mathscr{F}}^{t} y=\left\langle m_{1}^{y}, \ldots, m_{n}^{y}, y\right\rangle$. Hence, $x<_{\mathscr{F}}^{t} y$, by the Definition of $<_{\mathscr{F}}^{t}$.

But this contradicts the reductio assumption. Therefore, for every $x, y \in d_{\kappa, t} \cup$ $\mathscr{D}_{\kappa}$, for every $t \in \mathscr{T}: x<_{\mathscr{F}}^{t} y$ if $\theta(x)<_{\mathscr{F}}^{t} \theta(y)$.

We are now in a position to define the $<$-bisimulation $\pi$ :

\section{Definition 50 ( $\pi$ Function)}

Let $\pi$ be a function with domain $\mathscr{B}_{\kappa} \cup \cup_{n \in \mathbb{N}} \mathscr{D}_{\kappa}^{n} \cup \mathscr{T} \cup \mathscr{P}$ such that:

- $\forall x \in \mathscr{B}_{\kappa}: \pi(x)=\theta(x)$;

- $\forall \mathscr{X} \in \mathscr{D}_{\kappa}^{n}: \pi(\mathscr{X})=\theta(\mathscr{X})$;

- $\forall t \in \mathscr{T}: \pi(t)=t$

$-\forall p \in \mathscr{P}: \pi(p)=p$

The following lemma states that $\pi$ is indeed a $<$-bisimulation between $\mathscr{M}$ and $\mathscr{M}$ :

Lemma $17 \mathscr{M} \stackrel{\pi}{\leftrightarrows} \mathscr{M}$

Proof of Lemma 17 The satisfaction of conditions 1-9 of Definition 43 is a straightforward consequence of Definitions 49 and 50 and Lemmas A.2.2 and 16. Therefore, $\mathscr{M} \stackrel{\pi}{\leftrightarrows} \mathscr{M}$

The last result required for the application of Theorem 8 is the following:

\section{Lemma 18}

1. (a) $\mathscr{M}, 1, \mathcal{g}\left[x / a, y / e_{3}\right] \vDash x \ll y$

(b) $\mathscr{M}, \pi(1), \pi\left(g\left[x / a, y / e_{3}\right]\right) \not=x \ll y$;

2. (a) $\mathscr{M}, 1, q\left[x / a, y / e_{3}\right] \vDash x \triangleleft y$;

(b) $\mathscr{M}, \pi(1), \pi\left(g\left[x / a, y / e_{3}\right]\right) \not \forall x \triangleleft y$. 


\section{Proof of Lemma 18}

1. (a) Clearly, $a \ll_{\mathscr{F}}^{1} e_{3}$, by Definition 9 , since $\lambda t$. $a$ is the first element of $e_{3}=$ $\left\langle\lambda t . a, \lambda t . e_{1}, \lambda t . e_{2}, \mathscr{R}\right\rangle$ and $a=\lambda t . a(1)$ is such that $\left\langle a, e_{1}, e_{2}\right\rangle \in$ $\mathscr{R}(1)$; So, $\mathscr{M}, 1, \mathscr{Q}\left[x / a, y / e_{3}\right] \vDash x \ll y$;

(b) $\pi(a) \ll_{\mathscr{F}}^{\pi(1)} \pi\left(e_{3}\right)$ if and only if a $\ll_{\mathscr{F}}^{1} e_{4}$. Now, $e_{4}=$ $\left\langle\lambda t . b . \lambda t . e_{1}, \lambda t . e_{2}, \mathscr{X}\right\rangle$ and $a \neq \lambda t . \mathscr{b}(1)=b, a \neq \lambda t . e_{1}(1)=e_{1}$ and $a \neq \lambda t \cdot e_{2}(1)=e_{2}$. So, a $\ll_{\mathscr{F}}^{1} e_{4}$, by Definition 9. Hence, $\pi(a) K_{\mathscr{F}}^{\pi(1)} \pi\left(e_{3}\right)$. Therefore, $\mathscr{M}, \pi(1), \pi\left(g\left[x / a, y / e_{3}\right]\right) \not y x \ll y$

2. (a) Clearly, $a \triangleleft_{\mathscr{F}}^{1} e_{3}$, by Definition 13, since $a \ll \ll_{\mathscr{F}} e_{3}$ and $e_{3}$ is a rigid embodiment. So, $\mathscr{M}, 1, q\left[x / a, y / e_{3}\right] \vDash x \triangleleft y$;

(b) $\pi(a) \triangleleft_{\mathscr{F}}^{\pi(1)} \pi\left(e_{3}\right)$ if and only if $a \triangleleft_{\mathscr{F}}^{1} e_{4}$. The only immediate parthood sequence at time 1 linking $a$ to $e_{4}$ is $a \ll_{\mathscr{F}}^{1} e_{1} \ll_{\mathscr{F}}^{1} e_{4}$. But $e_{1}$ is not a rigid embodiment. So, not: $a \triangleleft_{\mathscr{F}}^{1} e_{4}$. Hence, not: $\pi(a) \triangleleft_{\mathscr{F}}^{\pi(1)} \pi\left(e_{3}\right)$, by Definition 13. So, $\mathscr{M}, \pi(1), \pi\left(g\left[x / a, y / e_{3}\right]\right) \not \forall x \triangleleft y$.

A straightforwardly corollary of the above results is the undefinability in the Etheory of timeless parthood and immediate parthood in terms of parthood. Theorem 2 is thus an immediate consequence of Theorem 8, Lemma 18 and Definitions 27 and 28.

Publisher's Note Springer Nature remains neutral with regard to jurisdictional claims in published maps and institutional affiliations.

\section{References}

1. Casati, R., \& Varzi, A.C. (1999). Parts and places: the structures of spatial representation. Cambridge: MIT Press.

2. Cotnoir, A.J. (2013). Beyond atomism. Thought, 2(1), 67-72.

3. Cotnoir, A.J. (2014). Universalism and junk. Australasian Journal of Philosophy, 92, $649-664$. https://doi.org/10.1080/00048402.2014.924540.

4. Cotnoir, A.J., \& Bacon, A. (2012). Non-wellfounded mereology. The Review of Symbolic Logic, 5(2), 187-204.

5. Evnine, S. (2016). Making objects and events: a hylomorphic theory of artifacts, actions, and organisms. Oxford: Oxford University Press.

6. Fairchild, M. (2017). A paradox of matter and form. Thought, 6, 33-42.

7. Fine, K. (1977). Properties, propositions and sets. Journal of Philosophical Logic, 6(1), 135-191.

8. Fine, K. (1982). Acts, events, and things. In Leinfellner, W., Kraemer, E., Schank, J. (Eds.) Language and ontology. Proceedings of the 6th international Wittgenstein symposium (pp. 97-105). Vienna: Hölder-Pichler-Tempsky.

9. Fine, K. (1994). Compounds and aggregates. Noûs, 28(2), 137-158.

10. Fine, K. (1999). Things and their parts. Midwest Studies in Philosophy, 23, 61-74.

11. Fine, K. (2005). Our knowledge of mathematical objects. In Gendler, T.Z., \& Hawthorne, J. (Eds.) Oxford studies in epistemology (pp. 89-109). Oxford: Clarendon Press.

12. Fine, K. (2006). Relatively unrestricted quantification. In Rayo, A., \& Uzquiano, G. (Eds.) Absolute Generality, chapter 2 (pp. 20-44). Oxford: Oxford University Press.

13. Fine, K. (2007). Response to Kathrin Koslicki. Dialectica, 61, 161-166. 
14. Fine, K. (2010). Towards a theory of part. The Journal of Philosophy, 107, 559-589.

15. Johnston, M. (2006). Hylomorphism. The Journal of Philosophy, 103(12), 652-698.

16. Koslicki, K. (2007). Towards a neo-Aristotelian mereology. Dialectica, 61(1), 127-159.

17. Koslicki, K. (2008). The structure of objects. Oxford: Oxford University Press.

18. Simons, P.M. (1987). Parts: a study in ontology. Oxford: Clarendon Press.

19. Varzi, A.C. (2008). The extensionality of parthood and composition. The Philosophical Quarterly, 58, $108-133$. 